\title{
Adventure in Topological Phase Transitions in 3 + 1-D: Non-Abelian Deconfined Quantum Criticalities and a Possible Duality
}

\author{
Zhen Bi and T. Senthil \\ Department of Physics, Massachusetts Institute of Technology, Massachusetts 02139, USA
}

(Received 2 January 2019; revised manuscript received 2 April 2019; published 20 May 2019)

\begin{abstract}
Continuous quantum phase transitions beyond the conventional paradigm of fluctuations of a symmetry-breaking order parameter are challenging for theory. These phase transitions often involve emergent deconfined gauge fields at the critical points as demonstrated in phase transitions between different broken-symmetry states of $2+1$-dimensional quantum magnets, as well as those between symmetry-protected topological (SPT) phases. In this paper, we present several examples of deconfined quantum critical points between SPT phases in $3+1-\mathrm{D}$ for both bosonic and fermionic systems. These critical theories can be formulated as non-Abelian gauge theories either in the infrared-free regime or in the conformal window when they flow to the Banks-Zaks fixed points. We explicitly demonstrate several interesting quantum critical phenomena. We describe situations in which the same phase transition allows for multiple universality classes controlled by distinct fixed points. We exhibit the possibility-which we dub "unnecessary quantum critical points"-of stable generic continuous phase transitions within the same phase. We present examples of interaction-driven, band-theory-forbidden, continuous phase transitions between two distinct band insulators. The understanding we develop leads us to suggest an interesting possible $3+1$-D field theory duality between $S U(2)$ gauge theory coupled to one massless adjoint Dirac fermion and the theory of a single massless Dirac fermion augmented by a decoupled topological field theory.
\end{abstract}

DOI: 10.1103/PhysRevX.9.021034

Subject Areas: Condensed Matter Physics,

Particles and Fields,

Topological Insulators

\section{INTRODUCTION}

Ground states of quantum many-particle systems can go through phase transitions as the Hamiltonian is tuned. When such a quantum phase transition is continuous, the resulting quantum critical point has many interesting properties, which have been explored for many decades $[1,2]$ in diverse contexts. Despite these studies, our intuition for what kinds of continuous quantum phase transitions are possible and their theoretical descriptions are very poor. The standard examples involve continuous quantum phase transitions separating a trivial gapped disordered phase from a symmetry-breaking phase with a Landau order parameter. In this case, the critical phenomena may be described within the framework of a quantum LandauGinzburg-Wilson (LGW) theory in terms of a fluctuating order parameter field.

Published by the American Physical Society under the terms of the Creative Commons Attribution 4.0 International license. Further distribution of this work must maintain attribution to the author(s) and the published article's title, journal citation, and DOI.
There are many examples of continuous quantum phase transitions that are beyond the Landau paradigm. First, one or both phases may have non-Landau order (for instance, they may have topological order). Then, since an orderparameter-based description fails to capture the nonLandau phase, it is not surprising that the critical theory is not within the standard LGW paradigm (see Ref. [3] for a review). Perhaps more surprisingly, Landau-forbidden continuous phase transitions may even occur between phases that are Landau allowed themselves. A classic example is the Néel-to-valence-bond solid-state transition of spin-1/2 quantum magnets on a $2 d$ square lattice [4-18]. The theory for this transition is an example of a phenomenon dubbed "deconfined quantum criticality." The critical field theory is conveniently expressed in terms of "deconfined" fractionalized degrees of freedom (d.o.f.), though the phases on either side only have conventional "confined" excitations. Now, there are many other proposed examples of deconfined quantum critical points in $2+1$ space-time dimensions [19-39]. Very similar (sometimes equivalent) theories emerge for critical points between trivial and symmetry-protected topological (SPT) phases of bosons in $2+1$ space-time dimensions [19,40-46]. (For a general introduction to SPT phases, see, e.g., Refs. [42,47-54].) 
(a)
Trivial gapped phase
Deconfined quantum
Symmetry Protected
critical point
Topological (SPT) phase

(b)

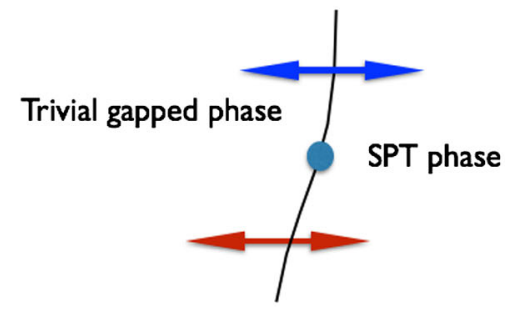

(c)

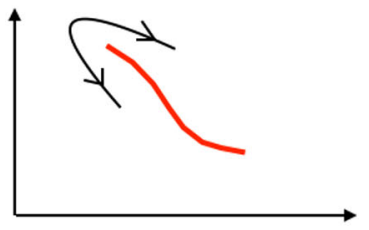

(d)

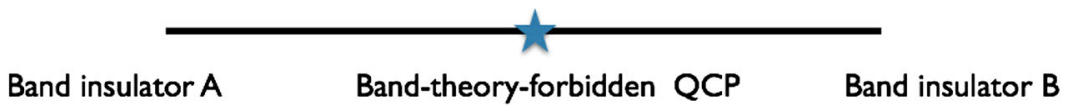

FIG. 1. (a) Deconfined quantum criticality at the trivial-to-SPT phase boundary of systems of either bosons or fermions. (b) Multiple universality classes for the same phase transition. (c) "Unnecessary quantum critical points" that live within a single phase of matter. (d) Band-theory-forbidden QCP between two band insulators.

These theories have been shown [19] to be related by webs of dualities of $2+1-\mathrm{D}$ conformal field theories discussed in recent years [55-58].

In this paper, we describe a number of surprising quantum critical phenomena for which there are no (or very few) previous examples as far as we know. Figure 1 contains a schematic description of some of our results. We construct examples of deconfined quantum critical points in $3+1-D$, for which there are no prior examples. These examples appear as critical theories separating trivial and SPT phases of either bosons or fermions. We describe situations where the same phase transition admits multiple universality classes depending on where the phase boundary is crossed. We also introduce and describe the concept of "unnecessary continuous phase transitions." These are continuous transitions that happen within the same phase. They are analogous to the liquid-gas transition except that they are continuous. When talking about quantum critical points in condensed matter physics, it is common to assume that the most fundamental question is to understand what the distinction is between the phases on either side of the transition. The existence of unnecessary continuous phase transitions shows that quantum critical points may occur that do not separate two distinct phases.

In fermionic systems, we describe examples of interaction-driven topological phase transitions that are not possible within a free fermion description, even though the phases themselves can be described by free fermions. These transitions violate band theory rules for which band insulators can be separated by continuous phase transitions. Such band-theory-forbidden continuous phase transitions between two band-theory-allowed phases of matter are a close fermionic analog of the familiar examples of Landau-forbidden continuous transitions between Landau-allowed phases in bosonic systems. We present examples where the critical theory is a deconfined gauge theory.

Many of our results are obtained by considering the phase diagram of non-Abelian gauge theories in space-time dimensions $\mathrm{D}=3+1$. If massless, we interpret the corresponding theory as a quantum critical point in the phase diagram and identify the nearby phases obtained by turning on relevant perturbations. As a bonus of the results on fermionic, deconfined, quantum critical points, we discuss a striking possible duality of fermions in 3+1-D. Specifically, we show that an $S U(2)$ gauge theory coupled to one massless adjoint Dirac fermion and massive fundamental bosons may share the same infrared (IR) physics with a theory of a free Dirac fermion supplemented by a gapped topological field theory. Both theories have the same local operators and the same global symmetries and anomalies. Furthermore, they support the same massive phases. These checks lend hope that the massless theories may also be infrared dual. Closely related work on $S U(2)$ gauge theories with adjoint fermions has recently appeared in Refs. [59,60], and we use some of their results. In $2+1-D$, dualities of Yang-Mills theories with adjoint fermions have been explored in recent work [61]. There are many famous examples of dualities of supersymmetric field theories in diverse dimensions [62]. Many interesting nonsupersymmetric dualities have been found in $2+1-\mathrm{D}$ 
(starting from old work [63-65] on charge-vortex duality in bosonic theories), particulary in recent years [55-58,6679]. However, there are no simple dualities of nonsupersymmetric theories that are known to us in $3+1-\mathrm{D}$.

\section{PRELIMINARIES AND SUMMARY OF RESULTS}

\section{A. Free massless Dirac fermions as quantum critical points}

In this section, we review how to interpret free massless Dirac fermion theories in space-time dimensions $D=3+1$ as quantum critical points. This review will enable us to introduce many ideas and methods that will be useful to us later in a simple setting.

Consider a free Dirac fermion described by the Lagrangian

$$
\mathcal{L}=\bar{\psi}(-i \not \partial+m) \psi
$$

Here, $\psi$ is a 4-component Dirac fermion. We regard this as the low-energy theory of electrons with global symmetry $U(1) \times Z_{2}^{T}$ (denoted as class AIII $[49,50]$ in the condensed matter literature). With this choice, the electric charge of the global $U(1)$ symmetry is odd under time reversal, $Z_{2}^{T}$. To probe the physics of the system, it is convenient to introduce a background $U(1)$ gauge field $A$ (more precisely, a $\operatorname{spin}_{c}$ connection [80]). We also place the theory on an arbitrary, smooth, oriented space-time manifold with metric $g$. Examining the partition function for arbitrary $(A, g)$ allows us to distinguish phases based on the response to these probes.

Consider the phase diagram as a function of the mass $m$. So long as $|m| \neq 0$, there is a gap in the spectrum. However, the phase with $m>0$ is distinct from the one with $m<0$. Taking the $m<0$ phase to be a trivial insulator [82], the $m>0$ phase will be a symmetryprotected topological insulator. Thus, the massless Dirac theory sits at a quantum critical point between a trivial and a topological insulator.

The topological distinction between the two phases can be understood physically by studying a domain wall in space where the mass $m$ changes sign. It is well known that at this domain wall, there is a single, massless, Dirac fermion, which reveals that the phase for one sign of the mass is topological when the other is trivial.

It is extremely useful to establish this result in a more formal but powerful way (see Ref. [83] for a review). Consider the partition function of the free Dirac theory defined by the Euclidean path integral:

$$
\begin{aligned}
Z[m ; A, g]= & \int \mathcal{D} \bar{\psi} \mathcal{D} \psi e^{-\int d^{4} x \sqrt{g} \bar{\psi}(\not D+m) \psi} \\
= & \operatorname{det}(\not D+m)
\end{aligned}
$$

Here, $D$ is a covariant derivative. As $\not D$ is anti-Hermitian, it has purely imaginary eigenvalues, which we write as $i \lambda_{i}$. Furthermore, as

$$
\left\{i \not D, \gamma^{5}\right\}=0
$$

for each nonzero eigenvalue $\lambda_{i}$, there is a partner $-\lambda_{i}$. Zero modes of the Dirac operator do not have to appear in pairs, however. These zero modes can be chosen to have definite helicity, i.e., to have $\gamma^{5}$ eigenvalues \pm 1 . Let $N_{ \pm}$be the number of zero modes with helicity \pm 1 , respectively. The index of the Dirac operator is defined to be $J=N_{+}-N_{-}$, and it is a topological invariant [it cannot be changed by smooth deformations of $(A, g)$ ]. Then, the partition function can be written

$$
Z[m ; A, g]=\left(\prod_{\lambda_{i}>0}\left(\lambda_{i}^{2}+m^{2}\right)\right)\left(m^{N_{+}+N_{-}}\right) .
$$

Now, consider the ratio of the partition functions of the theories with masses $+m$ and $-m$. Clearly,

$$
\frac{Z[m ; A, g]}{Z[-m ; A, g]}=(-1)^{N_{+}+N_{-}}=(-1)^{J}=e^{i \pi J} .
$$

Thus, the ratio of the partition functions is a topological invariant. Furthermore, it is known [81] (by the AtiyahSinger index theorem) that

$$
J=\frac{1}{2} \int d^{4} x \frac{F}{2 \pi} \wedge \frac{F}{2 \pi}-\frac{\sigma}{8},
$$

where $F=d A$ and $\sigma$ is an integer known as the signature of the space-time manifold. It may be expressed in terms of the Riemann curvature tensor:

$$
\sigma=-\frac{1}{24 \pi^{2}} \int d^{4} x \operatorname{tr}(R \wedge R) .
$$

Equation (6) thus gives exactly the right $\theta=\pi$ response of a topological insulator for one sign of mass if the other sign is chosen to be trivial.

We note that the massless Dirac theory has extra symmetries that are absent in the massive case. For instance, we can write the Dirac fermion as two flavors of Weyl fermions. The $S U(2)$ flavor rotation of the two Weyl fermions is a symmetry of the massless theory. We regard these symmetries as emergent symmetries of the critical point. These emergent symmetries have 't Hooft anomalies, and we discuss them later, as needed.

We can readily generalize the discussion above to $N$ free Dirac fermions or, equivalently, $2 N$ Majorana fermions with $S O(2 N) \times Z_{2}^{T}$ symmetry. Taking the $m<0$ theory to be trivial, the $m>0$ theory will describe a SPT phase of fermions with $S O(2 N) \times Z_{2}^{T}$ symmetry. This result is 
established by calculating the partition function ratio in the presence of a background $S O(2 N)$ gauge field $A^{S O(2 N)}$ and metric $g$ :

$$
\frac{Z\left[m ; A^{S O(2 N)}, g\right]}{Z\left[-m ; A^{S O(2 N)}, g\right]}=(-1)^{J}
$$

The index $J$ is a topological invariant related by the AtiyahSinger theorem to $\left(A^{S O(2 N)}, g\right)$ by

$$
2 J=p_{1}\left(A^{S O(2 N)}\right)-2 N \frac{\sigma}{8},
$$

where $p_{1}$ is the first Pontryagin index of the $S O(2 N)$ gauge field defined by

$p_{1}\left(A^{S O(2 N)}\right)=\frac{1}{2} \int_{Y_{4}} \operatorname{tr}_{S O(2 N)}\left(\frac{F^{S O(2 N)}}{2 \pi} \wedge \frac{F^{S O(2 N)}}{2 \pi}\right)$.

Therefore, $N$ massless free Dirac fermions can be viewed as the critical theory for the quantum phase transition between the trivial and SPT states of fermions with $S O(2 N) \times Z_{2}^{T}$ symmetry.

\section{B. Massless 3 + 1-D non-Abelian gauge theories}

Consider next a generalization to $S U(2)$ gauge theories coupled to $N_{f}$ flavors of fermionic matter fields. We study two distinct cases-(i) matter fields in the fundamental representation of $S U(2)$ and (ii) matter fields in the adjoint representation. These two distinct cases correspond "microscopically" to two very distinct kinds of physical situations. When the matter fields are in the fundamental representation, all local (i.e., gauge-invariant) operators in the theory (baryons, mesons, etc.) are bosons. We therefore regard the gauge theory as the low-energy theory of a UV theory of these gauge-invariant bosons [84]. When the matter fields are in the adjoint representation, however, there are local operators that are fermions. We can view the theory as emerging from a UV system of these fermions (see Sec. IV for more details).

The infrared behavior of $3+1-\mathrm{D}$ quantum chromodynamics with massless matter fields is an extremely important and intensively studied topic in particle physics. The renormalization group (RG) flow equation of the gauge coupling, for $S U\left(N_{c}\right)$ gauge theory with $N_{f}$ flavors of fermions [85] in the representation $R$, reads

$\beta\left(g^{2}\right)=\frac{d g^{2}}{d l}=\beta_{0}\left(N_{c}, N_{f}, R\right) g^{4}+\beta_{1}\left(N_{c}, N_{f}, R\right) g^{6}+O\left(g^{8}\right)$,

where $\beta_{0}$ and $\beta_{1}$ are functions that depend on $N_{c}, N_{f}$ and the representation $R$. For instance, if $R$ is the fundamental representation, $\beta_{0}$ and $\beta_{1}$ are

$$
\beta_{0}=\frac{1}{8 \pi^{2}} \frac{1}{3}\left(11 N_{c}-2 N_{f}\right)
$$

$$
\beta_{1}=\frac{1}{128 \pi^{2}}\left[\frac{34}{3} N_{c}^{2}-\frac{1}{2} N_{f}\left(2 \frac{N_{c}^{2}-1}{N_{c}}+\frac{20}{3} N_{c}\right)\right] .
$$

Based on the RG equation, the IR phases of the gauge theory can be divided into three classes. First, for $N_{f}$ bigger than a critical value $N_{1}\left(N_{c}, R\right)$, the leading term $\beta_{0}$ is negative $\left(\beta_{1}\right.$ is usually also negative for such $\left.N_{f}\right)$, and gauge coupling $g^{2}$ flows towards zero under RG, if we start from a weak initial coupling. In the IR, the theory is free, namely, decoupled gluons and free fermions. Second, for $N_{f}$ slightly smaller than the critical value $N_{1}, \beta_{0}$ is a small positive parameter. When we take into account the $g^{6}$ term in the RG equation, there is a stable fixed point controlled by $\epsilon=\beta_{0}$ at finite $g_{*}^{2} \sim O\left(\beta_{0} /\left|\beta_{1}\right|\right)$ for $\beta_{1}<0$. This point is the famous Bank-Zaks fixed point [86,87], which is an example of interacting conformal field theories in 3+1-D. As $N_{f}$ decreases further from $N_{1}$, in general, $\left|\beta_{1}\right|$ decreases and $g_{*}^{2}$ becomes larger. Eventually, for $N_{f}$ approaching a certain critical value $N_{2}\left(N_{c}, R\right),\left|\beta_{1}\right| \rightarrow 0$, and the fixed point goes to infinity, in which case, at low energy, the gauge theory is believed to be in a confined phase. The schematic RG flows for these three different regimes are summarized in Fig. 2(a). Naively, the critical $N_{2}$ can be estimated by solving the equation $\beta_{1}\left(N_{c}, N_{f}=N_{2}, R\right)=0$. However, at that point, perturbative $\mathrm{RG}$ is far from a controlled limit. Therefore, the value of $N_{2}$ is usually determined through numerical calculations. The gauge theory is in the conformal window if $N_{f} \in\left(N_{2}, N_{1}\right)$. The conformal windows are confirmed in numerical studies as well as in higher-loop calculations for $S U(2)$ gauge theories with fundamental fermions and adjoint fermions [88-92]. For fundamental fermions, the conformal window of $S U(2)$ theory is around 8 to 11 . For adjoint fermions, the conformal window is around 1 to 2 . One can find a plot for the conformal window of $S U\left(N_{c}\right)$ gauge theories in Fig. 2(b). The IR behavior of $\operatorname{Sp}\left(N_{c}\right)$ and $\operatorname{SO}\left(N_{c}\right)$ gauge theories is similar to that of $S U\left(N_{c}\right)$ gauge theories. Their corresponding conformal windows have also been discussed using various methods [91-94].

\section{Summary of results}

The IR-free gauge theories and the Bank-Zaks fixed points are interesting examples of $3+1$-D conformal field theories. In this paper, we show how to interpret them as quantum critical points in the phase diagram of the "microscopic" d.o.f. of the system, similar to what we reviewed for the free massless Dirac fermion theories in the previous section. Remarkably, we find that these theories can be viewed as deconfined quantum critical points for the underlying boson or fermion systems. The gauge-theory description emerges as a useful one right at the critical point 


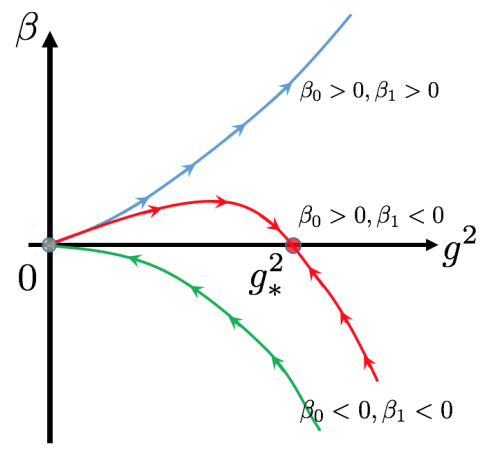

(a)

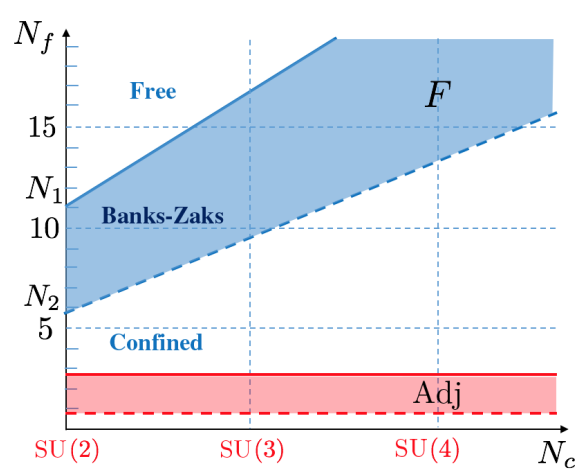

(b)

FIG. 2. (a) The renormalization group flow of the gauge coupling in three different regimes: (1) IR free (green curve), (2) Banks-Zaks fixed point (red curve), conformal, and (3) IR confined (blue curve). (b) The conformal window for $S U\left(N_{c}\right)$ gauge theories with $N_{f}$ flavors of fundamental or adjoint fermion fields. The upper edge of the conformal window is sharply determined by the condition $\beta_{0}\left(N_{c}, N_{f}, R\right)=0$. The lower edge of the conformal window can only be determined through numerical simulations and higher-loop calculations. Therefore, one should not take the numbers on the dotted line literally.

(and its vicinity), though the phases on either side only have conventional excitations (i.e., those that can be described simply in terms of the underlying bosons or fermions and their composites). In all cases we study, these massless gauge theories provide valuable examples of quantum critical points associated with phase transitions between trivial and SPT phases of the underlying boson or fermion system.

In Sec. III A, we describe 3 + 1-D deconfined quantum critical points for bosonic systems. We begin with $S U(2)$ gauge theory with $N_{f}$ fermions in the fundamental representation. For simplicity, we restrict our attention to $N_{f}$ even in this paper. We consider the theory in the presence of an arbitrary mass $m$ that preserves the flavor symmetry. When $m \neq 0$, the theory flows, in the IR, to massive phases. The $m=0$ point corresponds to a critical point. For general $m$, the global symmetry of the theory is [95] $\operatorname{PSp}\left(N_{f}\right) \times Z_{2}^{T}$. We regard this gauge theory as the IR theory of a system of UV (gauge-invariant) bosons with $P S p\left(N_{f}\right) \times Z_{2}^{T}$ global symmetry. First, consider $N_{f}$ large enough that the massless point is IR free. Thus, the gauge coupling $g^{2}$ flows to zero at the IR fixed point when $m=0$. For any $m \neq 0$, however, there is an induced effective action for the gauge field at low energies. The resulting pure $S U(2)$ gauge theory flows to strong coupling and will be confined at long length scales. In Fig. 3, we sketch the expected RG flows for this theory in the $g^{2}, m$ plane for large $N_{f}$. For even $N_{f}$ (the only case we consider), the confinement results in a trivial vacuum. Thus, the massless IR-free fixed point separates two strongly coupled confined

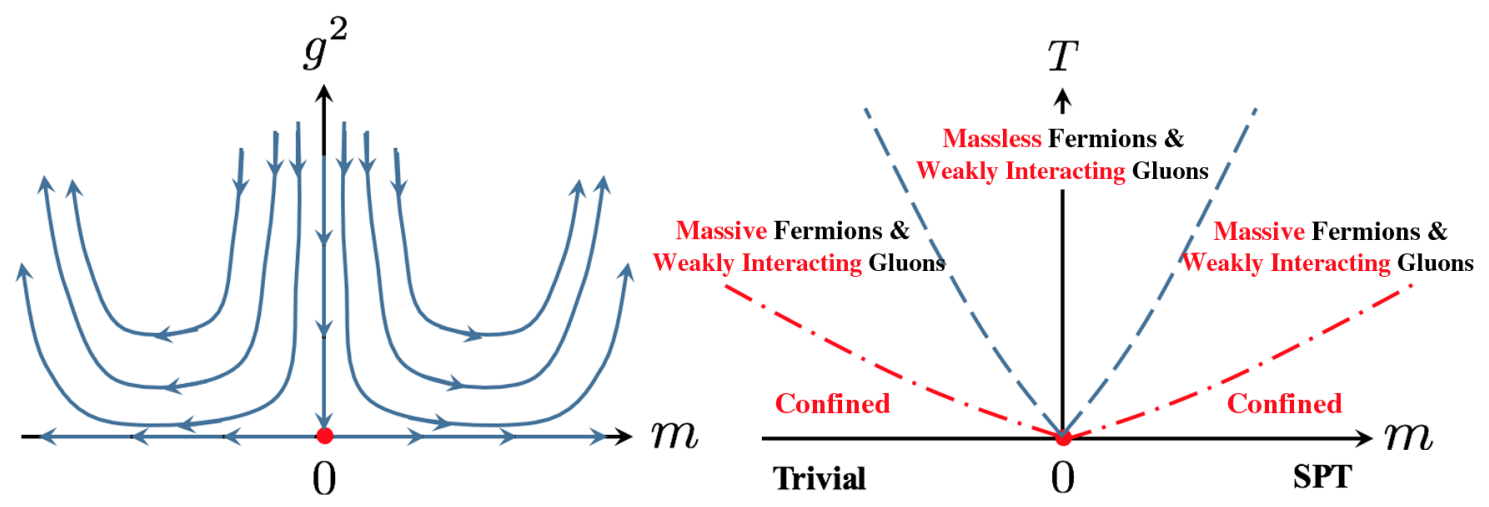

FIG. 3. On the left is a schematic demonstration of renormalization flow in the $g^{2}-m$ plane for large $N_{f}$ in the IR free case. The gauge coupling $g^{2}$ is a dangerous irrelevant operator for the $m=0$ critical point. On the right is the finite-temperature phase diagram for the deconfined quantum phase transition. It features two interesting crossover scales. At temperature $T \gg m$ (or length scale $l \ll \xi \sim 1 / m$ ), the physics is controlled by the critical point, and the system has deconfined massless fermions with weakly interacting gluons. For temperature $m^{y} \ll T \ll m$ (or length scale $\xi \ll l \ll \xi^{y}$ ) with $y>1$ a universal exponent, the system has deconfined but massive fermions and weakly interacting gluons. For temperature lower than about $m^{y}$ (or $l \gg \xi^{y}$ ), the gauge theory flows to strong coupling and the system is in a confined phase. 
phases with symmetric gapped ground states. However, we see that these phases are potentially distinct SPT phases of the underlying boson system with $\operatorname{PSp}\left(N_{f}\right) \times Z_{2}^{T}$ global symmetry. Just like in the free Dirac fermion, the massless theory has extra symmetries: We regard them as emergent symmetries of the massless fixed point and not as fundamental symmetries.

Note that the RG flows show that the Yang-Mills coupling $g^{2}$ is "dangerously irrelevant" in the vicinity of the massless fixed point. Naturally, there are then two length scales that emerge in the vicinity of the critical point. There is a first length scale $\xi \sim(1 / m)$ associated with the mass of the gauge charged fermions. At this scale, $g^{2}$ is still small. Confinement does not set in until a much larger second length scale $\xi_{\text {conf }} \sim \xi^{y}$, where $y>1$ is a universal exponent [96]. For $S U\left(N_{c}\right)$ gauge theory with $N_{f}$ fermion flavors in the fundamental representation, $y=\left[\left(2 N_{f}\right) /\left(11 N_{c}\right)\right]$. Close to the critical point, at length scales smaller than $\xi$, the physics is that of the IR-free massless fixed point of the large- $N_{f} S U(2)$ gauge theory. For length scales between $\xi$ and $\xi_{\text {conf }}$, the physics is that of massive fermions and massless gluons that are weakly interacting. Finally, at the longest length scales greater than $\xi_{\text {conf }}$, the physics is that of the trivial ground state of the underlying boson system (but potentially in a SPT phase). These critical crossovers are also manifested at nonzero temperature as two distinct temperature scales (see Fig. 3).

From a condensed matter perspective, consider systems of interacting bosons with $P S p\left(N_{f}\right) \times Z_{2}^{T}$ global symmetry; as we tune the parameters, we can drive phase transitions between the various SPT phases. From this point of view, as we show later, the $S U(2)$ gauge theory coupled to $N_{f}$ fundamental massless Dirac fermions emerges as a description of the quantum critical point between trivial and SPT states of bosons. The $S U(2)$ gauge fields only appear at the critical point. For $N_{f}<8$, the $S U(2)$ gauge theory is believed to be in a confined phase at low energy. This idea implies that either the phase transition is first order or there exists an intermediate, spontaneous symmetry-breaking phase separating the two SPT states. For $N_{f}>10$, the gauge theory provides a description of the continuous phase transition between the trivial and SPT states, where the critical point is free $S U(2)$ Yang-Mills theory with decoupled massless Dirac fermions. An interesting situation [97] is that, for $N_{f}=10$ and 8, the phase transition can be described by the Bank-Zaks fixed point, which is an interacting conformal field theory in 3+1-D.

In Sec. III B, we find generalizations of the above construction. The phase transitions between $\operatorname{PSp}\left(N_{f}\right) \times$ $Z_{2}^{T}$ bosonic SPT states can also be described by $\operatorname{Sp}\left(N_{c}\right)$ gauge theories coupled to $N_{f}$ fundamental massless Dirac fermions for any $N_{c}=4 \mathbb{Z}+1$. The transition is continuous provided that $N_{f}$ is inside or above the conformal window of $S p\left(N_{c}\right)$ gauge theories. These theories are weakly dual to the $S U(2)$ gauge theory described above in the sense that they are distinct low-energy descriptions of the same underlying UV physical system [in our case, bosons with global $\operatorname{PSp}\left(N_{f}\right) \times Z_{2}^{T}$ symmetry]. Furthermore, they describe the same phases and phase transitions of this system. However, clearly the theories with fixed $N_{f}$ and different $N_{c}$ are truly distinct conformal field theories. First, they clearly have different numbers of low-energy massless fields - this result may be formalized by computing their $a$ coefficients. Furthermore, the emergent symmetries (and their 't Hooft anomalies) of these theories at the massless point are different. Thus, these theories provide valuable examples where the same continuous phase transition admits multiple distinct universality classes, controlled by distinct fixed points. The IR theories are not dual in a strong sense, and they are distinct conformal field theories.

In Sec. III C, we discuss an interesting phenomenon, which we call unnecessary phase transitions. We define unnecessary phase transitions as generic continuous phase transitions within the same phase. We provide several explicit examples for this phenomenon. The first example is a bosonic system with $\operatorname{PSp}\left(N_{f}\right) \times Z_{2}^{T}$ symmetry at $N_{f}=4 \mathbb{Z}$. We show that there can be a generic continuous phase transition inside the topologically trivial phase of this bosonic system. The critical theory is an emergent $S p\left(N_{c}\right)$ gauge theory at $N_{c}=4 \mathbb{Z}$, with $N_{f}=4 \mathbb{Z}$ massless fundamental fermions. As the phases on the two sides of this critical point are identical, the transition can be bypassed by some symmetric path in the whole parameter space. However, the transition is locally stable. We give another example that does not involve emergent gauge fields. We consider 16 copies of a topological superconductor in the DIII class with an additional $S O(2) \times S O(7)$ global symmetry. In the topologically trivial phase of this system, there can exist a generic second-order transition characterized by 16 gapless free Majorana fermions in $3+1-D$. The transition can be circumvented by adding a strong interaction. In condensed matter physics, it is common that two phases separated by a discontinuous (i.e., first-order, as for the liquid-gas transition) phase transition can actually be the same phase. The examples in this section teach us that even a generic continuous phase transition does not necessarily change the nature of the state.

Sections IV and V contain examples of deconfined quantum critical points in fermionic systems for which there are very few previous examples. We study $3+1-\mathrm{D}$ fermionic deconfined quantum critical points that can be formulated as $S U(2)$ gauge theories coupled to $N_{f}^{A}$ flavors of adjoint Dirac fermions. The theory has local fermion operators (baryons), and we therefore regard it as a lowenergy theory of a microscopic system of these local fermions. However, to enable this point of view, we need 
to augment the theory by including a massive spin- $1 / 2$ [under the $S U(2)$ gauge transformation] scalar particle in our spectrum. Otherwise, the theory has physical loop d.o.f. corresponding to "electric" field lines in the spin- $1 / 2$ representation [98]. We call this massive spin-1/2 scalar the spectator field. To complete the theory, we need to specify its symmetry quantum numbers under the global symmetry, especially its time-reversal properties [99]. The adjoint $S U(2)$ theory can actually describe different quantum phase transitions depending on the time-reversal symmetry properties of the spectator field.

For $N_{f}^{A}>2$, the massless theory is free in the infrared limit. This theory, by tuning the fermion mass $m$, describes a quantum phase transition between a trivial and SPT state protected by the global symmetry, which is $S O\left(2 N_{f}^{A}\right) \times Z_{2}^{T}$. We first discuss the fermion SPT classification for this symmetry. For example, for $N_{f}^{A} \in 2 \mathbb{Z}+1$, we show that the classification is $\mathbb{Z}_{8} \times \mathbb{Z}_{2}$, generalizing the known results [100,101] for $S O(2) \times Z_{2}^{T}$ symmetry (known in the condensed matter literature as a class AIII topological superconductor). This generalization means that such systems form distinct SPT states labeled by a pair of integers $(n, \eta)$, where $n=0,1 ., \ldots, 7 \bmod 8$, and $\eta=0,1$ mod 2. Phases with $\eta=0$ are accessible within free fermion band theory. The IR-free massless gauge theory with $N_{f}^{A}>2$ sits at the critical point between two such SPT phases. A subtlety arises with the spectator field of the theory. The precise SPT phase changes depending on the time-reversal symmetry properties of the massive spectator field. With one choice of spectator field, it describes the phase transition between the $n=0, \eta=0$ (trivial) state and the $n=3, \eta=0$ SPT state. This is a quantum phase transition that is not generically second order in free fermion systems where $n$ can only jump by 1 . Thus, this is an example of an interaction-driven band-theoryforbidden quantum critical point between two band insulators. With a different choice of the spectator field, the adjoint $S U(2)$ theory can describe the phase transition between the trivial state and the $(n=-1, \eta=0)$ SPT state. This transition can also occur within band theory where it is described by a free Dirac theory of physical fermions. However, the gauge theory yields a distinct fixed point for the same transition. This case is yet another example of multiple universality classes for the same phase transition in fermionic systems. For $N_{f}^{A} \in 2 \mathbb{Z}$, the SPT phase on the $m>0$ side does not depend on the choice of spectator field.

If we banish the fundamental scalars from the spectrum, at the IR-free massless point, the 1 -form $\left(Z_{2}\right)_{1}$ symmetry is spontaneously broken. Turning on a small mass to the fermions confines the symmetry and restores the $\left(Z_{2}\right)_{1}$ symmetry. In other words, electric loops in the spin- $1 / 2$ representation are tensionful in the massive phase. These loops are decoupled from the physical excitations of this phase (which are the local fermions). Now, if we reintroduce the fundamental scalars, they will have no effect on the low-energy properties at the critical point. However, in the massive phase, the scalars allow the loops to break. At the same time, they also affect the SPT characterization of the phase.

In Sec. V, we consider the interesting case $N_{f}^{A}=1$ (augmented as above with a spectator fundamental scalar). This case describes the familiar system of fermions with $S O(2) \times Z_{2}^{T}$ symmetry (the class AIII topological superconductor). This theory is asymptotically free, and there is some numerical evidence showing that it flows to a CFT in the IR [89]. Therefore, we first consider the fate of this theory in the presence of a large mass (of either sign) when trivial confined phases indeed result. The precise SPT identification of these massive phases depends on the symmetry realization on the spectator boson in exactly the same way as for general $N_{f}^{A} \in 2 \mathbb{Z}+1$. In contrast to the previous examples, here the gauge-theory description of the massless point is strongly coupled. In Sec. VI, we explore the possibility of a low-energy theory that consists of a free Dirac fermion together with a decoupled topological field theory. This case may be viewed as a dual theory of the $S U(2)$ gauge theory with $N_{f}^{A}=1$ adjoint Dirac fermions [102]. The topological field theory is needed to match all the anomalies of the theory (in the absence of the spectator field) identified recently in Ref. [59]. We discuss physical properties of this topological order. We show that the free massless Dirac + topological theory has the same local operators and (almost) the same global symmetries (both exact and emergent), and it further enables matching all 't Hooft anomalies of the emergent symmetries in the UV gauge theory $[103,105,106]$. While these checks are necessary to claim a duality, they are not sufficient as a proof. A small mass in the gauge theory will map to a small mass of the physical Dirac fermions of the IR theory but will not destroy the extra topological order. This result leads to a situation where, between the two large mass insulators, there is an intermediate phase that has an additional topologically ordered sector.

Several mathematical details are given in the Appendixes. In particular, we present some simple models - not involving emergent gauge fields - for some of the phenomena depicted in Fig. 1. We describe an interesting mechanism for fractionalizing 1 -form $Z_{2}$ symmetry in topological field theories. We also briefly discuss some generalizations of the $S U(2)$ gauge theory with arbitrary flavors of adjoint Dirac fermions.

\section{BOSONIC DECONFINED CRITICAL POINTS IN 3 + 1-D}

In this section, we study quantum phase transitions between trivial and SPT phases in 3+1-D systems of interacting bosons. The critical theories we construct for 
such transitions resemble the features of deconfined quantum phase transitions in 2+1-D [4,19]. In particular, the critical point has emergent, non-Abelian, deconfined gauge fields and associated "fractionalized" matter fields. To understand a phase transition, it is often helpful to first identify the nature of the nearby phases, which provide crucial information about the critical fluctuations at the transition. Here, however, we pursue a reversed logic by asking the following question: Given some deconfined gauge theory in $3+1-\mathrm{D}$, what phase transition can this theory describe? To complete the phase diagram, we start from the deconfined gauge theory and then identify its nearby gapped phases by perturbing the theory with a relevant perturbation.

\section{A. $S U(2)$ gauge theory with $N_{f} \in 2 \mathbb{Z}$ fundamental fermions}

Consider $S U(2)$ gauge theory with $N_{f}$ Dirac fermions in the fundamental representation. We label it as $S U(2)+N_{f}^{F}$ theory. A key observation is that, in this theory, all local [i.e., $S U(2)$ gauge-invariant] operators are bosonic because they are composed of an even number of fundamental fermions [107]. Therefore, the theory describes a phase transition in a purely bosonic system.

A relevant perturbation that can drive the massless theory away from the critical point is the Dirac mass term, which is uniform for all flavors. The Lagrangian of the system can be written as follows:

$$
\mathcal{L}_{\mathrm{QCD}}=\sum_{i=1}^{N_{f}} i \bar{\psi}_{i} \gamma_{\mu} D_{\mu} \psi_{i}-m \bar{\psi}_{i} \psi_{i} .
$$

We first show that both $m<0$ and $m>0$ phases (at least for large $|m|$ ) are trivial gapped phases if $N_{f} \in 2 \mathbb{Z}$. Let us assume that in the $m<0$ phase, integrating out the massive fermions generates a trivial $\Theta$ term for the $S U(2)$ gauge theory. This case is always possible by certain UV regularization. Then, on the $m>0$ side, the massive fermions contribute a $\Theta$ term for the $S U(2)$ gauge field at $\Theta=\pi N_{f}$. With the condition $N_{f} \in 2 \mathbb{Z}$, both phases have trivial $S U(2) \Theta$ terms because of the $2 \pi$ periodicity of the $\Theta$ angle. Therefore, the $S U(2)$ gauge theory enters a trivial confined phase at low energy, and the system has a gapped spectrum in both cases. Importantly, it is believed that when pure $S U(2)$ gauge theory is confined, the resulting ground state is also topologically trivial: There is a unique ground state on all spatial manifolds. In condensed matter parlance, we expect a "short-rangeentangled" (SRE) ground state $[47,48]$. In contrast, if $N_{f} \in 2 \mathbb{Z}+1$, we have an $S U(2)$ gauge theory with $\Theta=$ $\pi$ for the $m>0$ phase. The dynamics of this gauge theory is nontrivial at low energy [108,109], and the ground state likely has long-range entanglement. To keep things simple in this paper, we henceforth focus on the case $N_{f} \in 2 \mathbb{Z}$.
With $N_{f} \in 2 \mathbb{Z}$, by tuning the uniform Dirac mass from negative to positive, the system goes between two gapped phases through a quantum phase transition, which is described by the massless $S U(2)+N_{f}^{F}$ theory. For large enough $N_{f}$, the IR physics of the $S U(2)+N_{f}^{F}$ theory is either free or controlled by the Bank-Zaks fixed point. Therefore, it describes a continuous phase transition. In the following, we explain that the $S U(2)+N_{f}^{F}$ theory with uniform Dirac mass has $P S p\left(N_{f}\right) \times Z_{2}^{T}$ symmetry. With this global symmetry, the uniform Dirac mass is the only symmetry-allowed relevant perturbation at the critical point. The $m<0$ and $m>0$ phases are the trivial and the symmetry-protected topological phases of this global symmetry, respectively.

In order to illustrate the global symmetry explicitly, let us construct the $S U(2)+N_{f}^{F}$ theory in a more systematic way. First, we consider $4 N_{f}$ flavors of Majorana fermions in $3+1-\mathrm{D}$,

$$
\mathcal{L}_{0}=\sum_{j=1}^{4 N_{f}} i \bar{\chi}_{j} \gamma_{\mu} \partial_{\mu} \chi_{j}-m \bar{\chi}_{j} \chi_{j}
$$

with $\left\{\gamma_{0}, \gamma_{1}, \gamma_{2}, \gamma_{3}\right\}=\left\{\sigma^{12}, i \sigma^{03},-i \sigma^{22},-i \sigma^{01}\right\}$ and $\bar{\chi}=$ $\chi^{T} \gamma_{0} \cdot \gamma_{5}=i \gamma_{0} \gamma_{1} \gamma_{2} \gamma_{3}=\sigma^{32}$. (Here, $\sigma^{i j}$ is the shorthand notation of $\sigma^{i} \otimes \sigma^{j}$.) At this stage, the system has an $S O\left(4 N_{f}\right)$ flavor symmetry and time-reversal symmetry $Z_{2}^{T}$, whose actions on the Majorana fields are as follows:

$$
S O\left(4 N_{f}\right): \chi_{i} \rightarrow O_{i j} \chi_{j}
$$

$\mathcal{T}: \chi_{i}(\mathbf{x}, t) \rightarrow \gamma_{0} \gamma_{5} \chi_{i}(\mathbf{x},-t)=-i \sigma^{20} \chi_{i}(\mathbf{x},-t), \quad i \rightarrow-i$.

It is easy to check that the $S O\left(4 N_{f}\right)$ and $Z_{2}^{T}$ symmetry [110] commute with each other and $\mathcal{T}^{2}=(-1)^{F}$. Next, we gauge a diagonal $S U(2)$ subgroup of the flavor symmetry. To specify the $S U(2)$ subgroup, we reorganize the fermion fields into a matrix form [19]. Let us split the Majorana flavor index into two indices, namely, labeling the Majorana fields as $\chi_{v, j}$, with $v=1,2, \ldots, N_{f}$ and $j=0,1,2,3$. The matrix fermion fields are defined as follows:

$$
X_{v, \alpha ; \beta}=\frac{1}{\sqrt{2}}\left(\chi_{v, 0} \mathbb{I}_{\alpha \beta}+i \sum_{\mu=1}^{3} \chi_{v, \mu} \sigma_{\alpha \beta}^{\mu}\right),
$$

where the $\sigma^{\mu}$ 's are Pauli matrices and $\alpha, \beta=1,2$. This step can be viewed as combining four real fields into one quaternion field. The theory written in terms of $X$ is manifestly invariant under right $S U(2)$ rotation and a left unitary rotation, 


$$
X \rightarrow L X R_{S U(2)} .
$$

The left rotation $L$ must satisfy the reality condition of Majorana fermions. As a result, $L$ actually belongs to the $S p\left(N_{f}\right)$ group. It turns out that the $S p\left(N_{f}\right)$ group is the maximal symmetry group that commutes with the $S U(2)$. The $S U(2)$ and $S p\left(N_{f}\right)$ share the same center symmetry, namely, $S O\left(4 N_{f}\right) \supset\left\{\left[S U(2) \times S p\left(N_{f}\right)\right] / Z_{2}\right\}$. We now gauge the $S U(2)$ symmetry and get our $S U(2)+N_{f}^{F}$ theory,

$$
\mathcal{L}_{\mathrm{QCD}}=\operatorname{tr}\left(i \bar{X} \gamma_{\mu} D_{\mu} X-m \bar{X} X\right),
$$

where $\bar{X}=X^{\dagger} \gamma_{0}$. We can map this formulation back to the complex Dirac fermions in Eq. (15) by $\psi_{\alpha, i}=i \sigma_{\alpha, \beta}^{y} X_{1, i ; \beta}$, where $\alpha$ is the $S U(2)$ index and $i$ the flavor index.

The global symmetry after gauging the $S U(2)$ subgroup is manifestly $\mathcal{G}=\operatorname{PSp}\left(N_{f}\right) \times Z_{2}^{T}$. One can check that with this global symmetry, the uniform Dirac mass is the only allowed mass term. For example, the $i m \bar{X} \gamma_{5} X$ mass is not time-reversal invariant. Any mass term of the form $\bar{\chi}_{i} S_{i j} \chi_{j}$ or $i \bar{\chi}_{i} \gamma_{5} S_{i j} \chi_{j}$, with $S_{i j}=S_{j i}$, is not invariant under $\operatorname{PSp}\left(N_{f}\right)$ rotation.

In the two gapped phases, on any closed spatial manifold, the system has a nondegenerate ground state and no spontaneous symmetry breaking. The distinction of the two phases can only come from their topological properties. They can be different SPT phases of the global symmetry $\mathcal{G}$. Let us assume that the $m<0$ phase is the trivial disordered phase under this symmetry. Now, we want to understand the nature of the $m>0$ phase. The strategy is to couple the system to the background gauge fields of the global symmetry $\operatorname{PSp}\left(N_{f}\right)$ and identify its topological response, which is a signature of the SPT state. To achieve this, we first turn on a background gauge field for the whole $S O\left(4 N_{f}\right)$ flavor group and find its topological response. Then, we reduce the response theory down to its $S U(2)$ and $\operatorname{PSp}\left(N_{f}\right)$ subgroups.

Let us start from Eq. (16) and turn on a background $S O\left(4 N_{f}\right)$ gauge field $A^{S O\left(4 N_{f}\right)}$. We consider the response to the $S O\left(4 N_{f}\right)$ gauge field after integrating out the massive fermions. As shown in the previous sections, the ratio between the Euclidean partition functions with $m<0$ and $m>0$ is a good way to formulate the topological response. From Eqs. (9) and (10), we get

$$
\begin{aligned}
\mathcal{S}_{\text {topo }} & =\log \left\{\frac{\mathcal{Z}[m<0, A, g]}{\mathcal{Z}[m>0, A, g]}\right\} \\
& =i \frac{\pi}{2}\left(p_{1}\left(A^{S O\left(4 N_{f}\right)}\right)-\frac{N_{f}}{2} \sigma\right) .
\end{aligned}
$$

The topological action contains the $\Theta$ terms of the $S O\left(4 N_{f}\right)$ gauge field in terms of the first Pontryagin class $p_{1}$ and the gravitational $\Theta$ term [written in terms of the manifold signature $\sigma$-see Eq. (8)]. The first Pontryagin class is written as

$$
\begin{aligned}
p_{1}\left(A^{S O\left(4 N_{f}\right)}\right) & =\frac{1}{2} \int_{Y_{4}} \operatorname{tr}_{S O\left(4 N_{f}\right)}\left(\frac{F^{S O\left(4 N_{f}\right)}}{2 \pi} \wedge \frac{F^{S O\left(4 N_{f}\right)}}{2 \pi}\right) \\
& =2 l_{S O\left(4 N_{f}\right)},
\end{aligned}
$$

and it is equal to twice the instanton number of the $S O\left(4 N_{f}\right)$ gauge field. More details about the definition for the Pontryagin class and instanton number are given in Appendix A.

We restrict the $S O\left(4 N_{f}\right)$ to particular configurations that have separate $S p\left(N_{f}\right)$ and $S U(2)$ gauge fields.

$$
\begin{gathered}
p_{1}\left(A^{S O\left(4 N_{f}\right)}\right)=2 p_{1}\left(A^{S p\left(N_{f}\right)}\right)+2 N_{f} p_{1}\left(a^{S U(2)_{g}}\right), \\
=2 l_{S p\left(N_{f}\right)}+2 N_{f} l_{S U(2)}, \\
=2 l_{P S p\left(N_{f}\right)}+2 N_{f} l_{S O(3)}, \\
=\mathcal{P}\left(w_{2}^{P S p\left(N_{f}\right)}\right)+2 w_{4}^{P S p\left(N_{f}\right)}+\frac{N_{f}}{2} \mathcal{P}\left(w_{2}^{S O(3)}\right) \bmod 4,
\end{gathered}
$$

where $l$ represents the instanton number for the gauge bundle, $\mathcal{P}(a)$ is the Pontryagin square operator (for a definition, see Refs. $[19,111]$ and references therein), and $w_{2}$ and $w_{4}$ are the second and fourth Stiefel-Whitney classes [81]. Here, we use the following relations between the instanton numbers and characteristic classes for the vector bundles $[19,112]$ :

$2 l_{P S p\left(N_{f}\right)}=\mathcal{P}\left(w_{2}^{P S p\left(N_{f}\right)}\right)+2 w_{4}^{P S p\left(N_{f}\right)} \bmod 4$ for $N_{f} \in 2 \mathbb{Z}$,

$$
4 l_{S O(3)}=\mathcal{P}\left(w_{2}^{S O(3)}\right) \bmod 4 .
$$

Since our fermions transform projectively under the $S O(3)$ and $P S p\left(N_{f}\right)$ gauge bundle, in order for the theory to be consistently defined on any manifold with or without spin structure, we should impose the following constraint on the gauge bundles:

$$
w_{2}^{S O(3)}+w_{2}^{P S p\left(N_{f}\right)}+w_{2}^{T Y_{4}}=0 \bmod 2 .
$$

This constraint is the obstruction-free condition to lift a $S O(3) \times P S p\left(N_{f}\right) \times S O(4)^{T Y_{4}}$ bundle to $\left(S U(2) \times S p\left(N_{f}\right) \times\right.$ $\left.\operatorname{Spin}(4)^{T Y_{4}}\right) /\left(\mathbb{Z}_{2} \times \mathbb{Z}_{2}\right)$. Based on this relation and the following few useful identities (for references, see Wang et al. [19]),

$$
\mathcal{P}(a+b)=\mathcal{P}(a)+\mathcal{P}(b)+2 a \cup b \bmod 4,
$$




$$
\begin{gathered}
\mathcal{P}(a)=a \cup a \bmod 2, \\
a \cup w_{2}^{T Y_{4}}=a \cup a \quad \text { for any } a \in H^{2}\left(Z_{2}\right), \\
\int_{Y_{4}} \mathcal{P}\left(w_{2}^{T Y_{4}}\right)=\sigma \bmod 4,
\end{gathered}
$$

we can simplify the response theory in Eq. (27). There are four types of response theories depending on $N_{f} / 2=k$ $\bmod 4$

(i) $k=0 \bmod 4$, e.g., $N_{f}=8,16,24, \ldots$

$$
S_{\text {topo }}^{k=1}=i \pi l_{P S p\left(N_{f}\right)} .
$$

This result is the usual $\Theta$ term for the $\operatorname{PSp}\left(N_{f}\right)$ gauge field, and the value of $\Theta=\pi$ is protected by $Z_{2}^{T}$ symmetry.

(ii) $k=1 \bmod 4$, e.g., $N_{f}=2,10,18, \ldots$

$$
S_{\text {topo }}^{k=1}=i \pi w_{4}^{P S p\left(N_{f}\right)} .
$$

The topological phase associated with such topological response is robust against $Z_{2}^{T}$ breaking because $w_{4}$ is a $\mathbb{Z}_{2}$ class. However, if $Z_{2}^{T}$ is broken, the $\bar{\psi} i \gamma_{5} \psi$ mass is also allowed at the critical point. Therefore, the $Z_{2}^{T}$ symmetry must be preserved in order to have a generic second-order transition.

(iii) $k=2 \bmod 4$, e.g., $N_{f}=4,12,20, \ldots$

$S_{\text {topo }}^{k=2}=i \pi l_{P S p\left(N_{f}\right)}+i \pi w_{2}^{P S p\left(N_{f}\right)} \cup w_{2}^{P S p\left(N_{f}\right)}$.

The first term is the $\Theta$ term for the $P \operatorname{Sp}\left(N_{f}\right)$ gauge fields, which requires $Z_{2}^{T}$ symmetry to be stable. The second term is an independent topological term that can be nontrivial on a nonspin manifold. The second term is a $\mathbb{Z}_{2}$ class and hence is stable against $Z_{2}^{T}$ breaking.

(iv) $k=3 \bmod 4$, e.g., $N_{f}=6,14,22, \ldots$

$$
S_{\text {topo }}^{k=3}=i \pi w_{4}^{P S p\left(N_{f}\right)}+i \pi w_{2}^{P S p\left(N_{f}\right)} \cup w_{2}^{P S p\left(N_{f}\right)} .
$$

Both terms are stable against $Z_{2}^{T}$ symmetry breaking. The conformal window for $S U(2)+N_{f}^{F}$ theory is $N_{f} \sim 6-11$ from numerical studies. For $N_{f}>11$, the theory is free. Therefore, we have many examples of $3+1-\mathrm{D}$ deconfined quantum phase transitions between the trivial and the $\operatorname{PSp}\left(N_{f}\right) \times Z_{2}^{T}$ SPT states, which are described by free $S U(2)+N_{f}^{F}$ theory for even $N_{f}>11$. Assuming further that for $N_{f}=8$ and 10 a small mass can drive the Banks-Zaks theories to the large mass fixed points, we then have two explicit examples of $3+1-D$

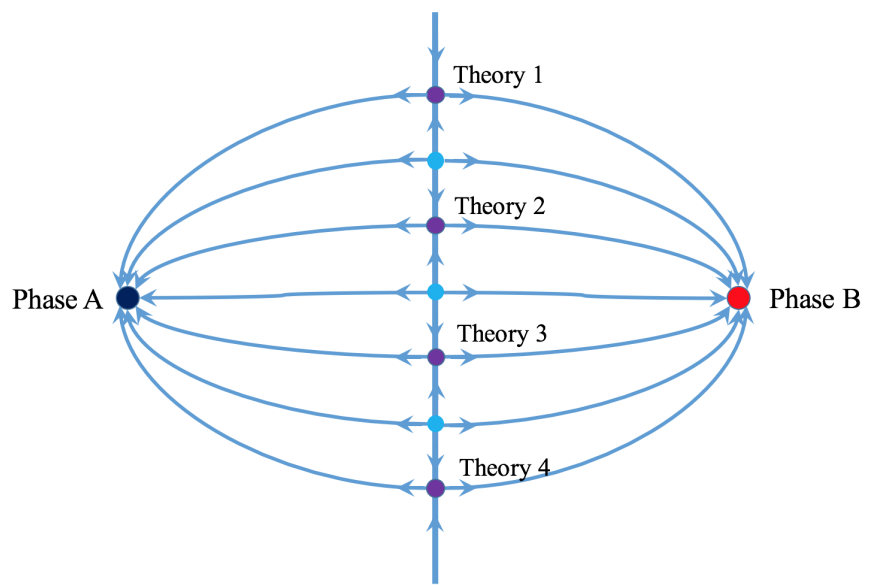

FIG. 4. A schematic renormalization flow diagram for degenerate quantum critical points.

DQCP, which are described by strongly interacting CFTs. They separate trivial and the $P S p\left(N_{f}\right) \times Z_{2}^{T}$ bosonic SPT states.

\section{B. Multiple universality classes}

In this section [113], we demonstrate that the phase transition between the trivial and SPT states protected by $P S p\left(N_{f}\right) \times Z_{2}^{T}$ symmetry can have many descriptions that are distinct from each other. A schematic renormalization flow diagram is shown in Fig. 4. In practice, such a situation, although not forbidden by any physical principle, is not commonly observed in critical phenomena. It is interesting that here we can show such an example explicitly in a controlled way.

To introduce these different transition theories, we consider a generalization of our previous construction of $3+1$-D bosonic DQCP. We start with $4 N_{c} N_{f}$ flavors of a Majorana fermion in $3+1$ dimensions,

$$
\mathcal{L}_{0}=\sum_{j=1}^{4 N_{c} N_{f}} \bar{\chi}_{j}\left(i \gamma_{\mu} \partial_{\mu}-m\right) \chi_{j}
$$

The total flavor symmetry is $S O\left(4 N_{c} N_{f}\right)$. There is a wellknown group decomposition for the $S O\left(4 N_{c} N_{f}\right)$ group,

$$
S O\left(4 N_{c} N_{f}\right) \supset \frac{S p\left(N_{c}\right) \times S p\left(N_{f}\right)}{Z_{2}} .
$$

We can understand the general group decomposition intuitively as follows. First, we use four real fermions to form a quaternion fermion. Then, we arrange the $N_{f} N_{c}$ quaternion fermions into a $N_{f} \times N_{c}$ quaternion matrix fermion field $\mathcal{X}$. The $S p\left(N_{f}\right)$ transformation can be packed into a $N_{f} \times N_{f}$ quaternion matrix $\mathcal{L}$, and it has a natural action on an $N_{f}$-dimensional quaternion vector. Thus, the 
$S p\left(N_{f}\right)$ action on the $\mathcal{X}$ field is the left multiplication on the $\mathcal{X}$ matrix, namely, $\mathcal{X} \rightarrow \mathcal{L} \mathcal{X}$. Similarly, the $\operatorname{Sp}\left(N_{c}\right)$ action is the right multiplication on $\mathcal{X}$ by an $N_{c} \times N_{c}$ quaternion matrix $\mathcal{R}$, namely, $\mathcal{X} \rightarrow \mathcal{X} \mathcal{R}$ [114]. The group decomposition we used in the previous section is a special case with $N_{c}=1$ and $N_{f} \in 2 \mathbb{Z}$.

Let us gauge the $S p\left(N_{c}\right)$ part of the flavor symmetry. The result is an $\operatorname{Sp}\left(N_{c}\right)$ gauge theory with $N_{f}$ fundamental fermions, which we label as $\operatorname{Sp}\left(N_{c}\right)+N_{f}^{F}$ theory,

$$
\mathcal{L}_{\mathrm{QCD}}=\operatorname{tr}\left(i \overline{\mathcal{X}} \gamma_{\mu} D_{\mu} \mathcal{X}-m \overline{\mathcal{X}} \mathcal{X}\right)
$$

The global symmetry of this theory is again $\operatorname{PSp}\left(N_{f}\right) \times$ $Z_{2}^{T}$. Notice the global symmetry only depends on $N_{f}$ but not on $N_{c}$. Next, we need to identify the nature of the $m<0$ and $m>0$ phases by their topological response to the background field of the global $\operatorname{PSp}\left(N_{f}\right)$ symmetry. After integrating out fermions, we get the following topological action for the $m>0$ phase:

$$
\begin{aligned}
\mathcal{S}_{\text {topo }} & =i \frac{\pi}{2}\left(2 l_{S O\left(4 N_{c} N_{f}\right)}-\frac{4 N_{c} N_{f}}{8} \sigma\right), \\
& =i \frac{\pi}{2}\left(2 N_{c} l_{P S p\left(N_{f}\right)}+2 N_{f} l_{P S p\left(N_{c}\right)}-\frac{N_{c} N_{f}}{2} \sigma\right) .
\end{aligned}
$$

The instanton numbers have the following algebraic relations with the Stiefel-Whitney classes [112]. For $n \in \mathbb{Z}$,

$$
\begin{gathered}
4 l_{P S p(2 n+1)}=\mathcal{P}\left(w_{2}^{P S p(2 n+1)}\right) \bmod 4, \\
2 l_{P S p(2 n)}=\mathcal{P}\left(w_{2}^{P S p(2 n)}\right)+2 w_{4}^{P S p(2 n)} \bmod 4 .
\end{gathered}
$$

Let us consider a case in which $N_{f}=2 p, p \in \mathbb{Z}, N_{c}=$ $4 q+1, q \in \mathbb{Z}$. With the above relations, we can simplify the topological action.

$$
\begin{aligned}
\mathcal{S}_{\text {topo }}= & i \frac{\pi}{2}\left\{2 N_{c} l_{P S p\left(N_{f}\right)}+2 N_{f} l_{P S p\left(N_{c}\right)}-\frac{N_{c} N_{f}}{2} \sigma\right\}, \\
= & i \frac{\pi}{2}\left\{(4 q+1)\left(\mathcal{P}\left(w_{2}^{P S p\left(N_{f}\right)}\right)+2 w_{4}^{P S p\left(N_{f}\right)}\right)\right. \\
& \left.+p \mathcal{P}\left(w_{2}^{P S p\left(N_{c}\right)}\right)-p(4 q+1) \sigma\right\}, \\
= & i \frac{\pi}{2}\left\{\mathcal{P}\left(w_{2}^{P S p\left(N_{f}\right)}\right)+2 w_{4}^{P S p\left(N_{f}\right)}\right. \\
& \left.+p \mathcal{P}\left(w_{2}^{P S p\left(N_{c}\right)}\right)-p \sigma\right\} .
\end{aligned}
$$

If $p \in 4 \mathbb{Z}+1$, namely, $N_{f} \in 8 \mathbb{Z}+2$, we get $\mathcal{S}_{\text {topo }}=i \frac{\pi}{2}\left\{\mathcal{P}\left(w_{2}^{P S p\left(N_{f}\right)}\right)+2 w_{4}^{P S p\left(N_{f}\right)}+\mathcal{P}\left(w_{2}^{P S p\left(N_{c}\right)}\right)-\sigma\right\}$,

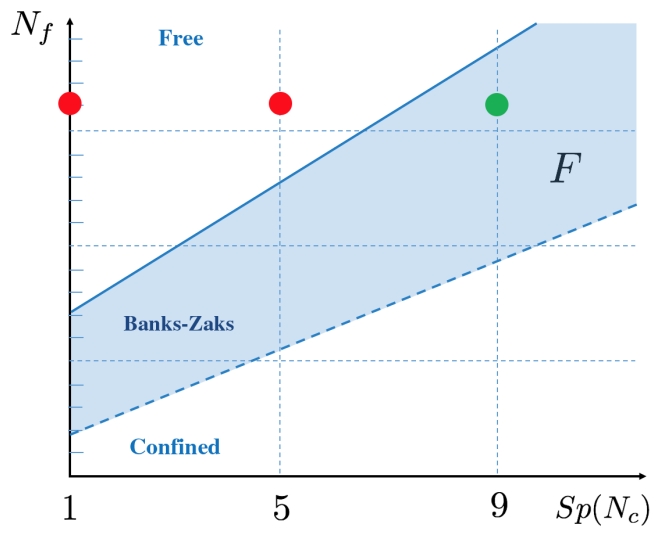

FIG. 5. A sketch for the conformal window of $S p\left(N_{c}\right)$ gauge theories (numbers on the $N_{f}$ axis are not precise). The red and green dots are different gauge theories. The red ones are free, and the green one is strongly interacting. However, they all describe the topological phase transition from the trivial state to the same $P S p\left(N_{f}\right) \times Z_{2}^{T}$ bosonic symmetry-protected topological phase.

$$
=i \pi w_{4}^{P S p\left(N_{f}\right)}+i \frac{\pi}{2}\left\{\mathcal{P}\left(w_{2}^{P S p\left(N_{f}\right)}\right)+\mathcal{P}\left(w_{2}^{P S p\left(N_{c}\right)}\right)-\sigma\right\} .
$$

We have the following consistency relation for the gauge and tangent bundles, which is the analog of Eq. (30),

$$
w_{2}^{P S p\left(N_{f}\right)}+w_{2}^{P S p\left(N_{c}\right)}+w_{2}^{T Y}=0 \bmod 2 .
$$

We can prove that the second term in Eq. (50) vanishes $\bmod 4$.

$$
\begin{aligned}
& \mathcal{P}\left(w_{2}^{P S p\left(N_{f}\right)}\right)+\mathcal{P}\left(w_{2}^{P S p\left(N_{c}\right)}\right)-\sigma \\
& =2 \mathcal{P}\left(w_{2}^{P S p\left(N_{c}\right)}\right)+2 w_{2}^{P S p\left(N_{c}\right)} \cup w_{2}^{T Y}+\mathcal{P}\left(w_{2}^{T Y}\right)-\sigma \\
& =0 \bmod 4 .
\end{aligned}
$$

In the derivation, we have again used relations in Eqs. (31)(34) to simplify the result. In the end, the topological response for the background $\operatorname{PSp}\left(N_{f}\right)$ gauge field is quite simple and familiar. The topological action reads

$$
\mathcal{S}_{\text {topo }}=i \pi w_{4}^{P S p\left(N_{f}\right)} \text { for } N_{c} \in 4 \mathbb{Z}+1, \quad N_{f} \in 8 \mathbb{Z}+2 .
$$

One interesting observation is that the topological action does not depend on $N_{c}$ as long as $N_{c} \in 4 \mathbb{Z}+1$. For a fixed but very large $N_{f} \in 8 \mathbb{Z}+2$ and small enough $N_{c} \in 4 \mathbb{Z}+1$, the $\operatorname{Sp}\left(N_{c}\right)+N_{f}^{F}$ theory is free in the infrared limit. By increasing $N_{c} \in 4 \mathbb{Z}+1$ before it hits some critical value, we have different free $S p\left(N_{c}\right)$ gauge theories. (They are labeled by the red dots in Fig. 5.) Most importantly, these theories all describe a phase transition between the 
trivial state and the same SPT state protected by $\operatorname{PSp}\left(N_{f}\right) \times Z_{2}^{T}$ symmetry.

These free theories are truly distinct conformal field theories. For instance, they have different numbers of emergent low-energy d.o.f. This distinction may be formalized based on the $a$ theorem. The quantity $a$ is a universal number used to characterize 4D CFT. It is the 4D analogy of the central charge of 2D conformal field theories. The trace of the stress energy tensor of a 4D CFT can be expressed as follows:

$$
\left\langle T_{\mu}^{\mu}\right\rangle=-a E_{4}+c W^{2},
$$

where $E_{4}$ is the Euler density and $W^{2}$ is the square of the Weyl tensor. The $a$ value is a universal signature for a 4D CFT, similar to the central charge of 2D CFTs. It was conjectured and subsequently proven to be a monotonic function under RG flow [116], the so-called $a$ theorem. Since these $\operatorname{Sp}\left(N_{c}\right)+N_{f}^{F}$ theories are IR-free theories, we know the answer for the $a$ values [116],

$$
a\left(N_{c}, N_{f}\right)=22 N_{c} N_{f}+62 N_{c}\left(2 N_{c}+1\right) .
$$

For fixed $N_{f}$, different $N_{c}$ 's give different $a$ values, indicating that they are distinct 4D CFTs.

Furthermore, if $N_{c}$ is in an appropriate range, the $S p\left(N_{c}\right)+N_{f}^{F}$ theory can possibly fall into the conformal window of $\operatorname{Sp}\left(N_{c}\right)$ gauge theory (labeled by the green dots in Fig. 5), which is described by the Bank-Zaks fixed point. It is a strongly interacting deconfined gauge theory, which is most likely distinct from free theories. For instance, it has different scaling dimensions for the gauge-invariant operators from those of the free theories [88].

The $\operatorname{Sp}\left(N_{c}\right)$ generalization provides an explicit example for the phenomenon that there can exist multiple distinct critical theories that describe the transition between the same two nearby phases. In this controlled example, we are certain that these critical points are not dual to each other. We call them multiversality classes. In later sections, we provide more examples of such phenomena for fermionic deconfined critical points.

\section{Unnecessary continuous phase transitions}

In this section, we introduce a phenomenon that we call the unnecessary phase transition. Unnecessary phase transitions are generic, stable, continuous phase transitions between two identical phases. We show examples of such a phenomenon within the $S p\left(N_{c}\right)+N_{f}^{F}$ theory. We also discuss examples that do not involve gauge fields.

Example 1: $\quad S p\left(N_{c}\right)+N_{f}^{F}$ theory with $N_{c} \in 4 \mathbb{Z}$ and $N_{f} \in 4 \mathbb{Z}$.

The first example we consider is the $S p\left(N_{c}\right)+N_{f}^{F}$ theory with different $N_{c}$ and $N_{f}$ from previous sections.

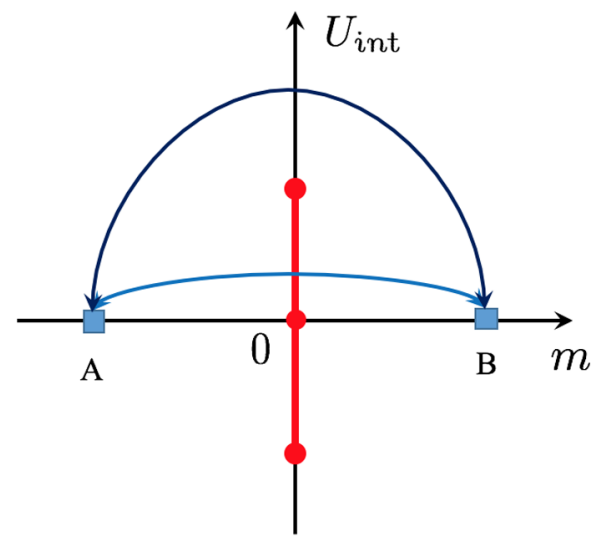

FIG. 6. A schematic phase diagram for unnecessary phase transitions.

An interesting situation is $N_{c}=4 q \in 4 \mathbb{Z}$ and $N_{f}=$ $4 p \in 4 \mathbb{Z}$. With such conditions, the two phases with $m<0$ and $m>0$ are actually the same phase. We can show that the topological response for the $m>0$ phase is $\mathcal{S}_{\text {topo }}=i 2 \pi \mathbb{Z}$,

$$
\begin{aligned}
& \mathcal{S}_{\text {topo }}= i \frac{\pi}{2}\left(2 N_{c} l_{P S p\left(N_{f}\right)}+2 N_{f} l_{P S p\left(N_{c}\right)}-\frac{4 N_{c} N_{f}}{8} \sigma\right) \\
&= i \frac{\pi}{2}\left\{4 q\left(\mathcal{P}\left(w_{2}^{P S p\left(N_{f}\right)}\right)+2 w_{4}^{P S p\left(N_{f}\right)}\right)\right. \\
&\left.+4 p\left(\mathcal{P}\left(w_{2}^{P S p\left(N_{c}\right)}\right)+2 w_{4}^{P S p\left(N_{c}\right)}\right)-\frac{16 q p}{2} \sigma\right\} \\
& \sim 2 \pi i \mathbb{Z} .
\end{aligned}
$$

Namely, $m<0$ and $m>0$ have identical partition functions for any gauge configuration, which means the two phases are identical.

Nevertheless, there is a generic continuous phase transition in the phase diagram by tuning $m$ from negative to positive. In the large- $N_{f}$ limit, the $m=0$ theory is IR free. The uniform mass $m$, as the driving parameter, is the only relevant symmetric perturbation at the critical point. Other generic interactions that respect the symmetry are perturbatively irrelevant. In other words, the IR free gauge theory controls the whole critical line that exists within a single phase of matter. A schematic phase diagram is shown in Fig. 6 [117].

Example 2: 16 copies of ${ }^{3} \mathrm{He}-B$ phases. We now describe an example for an unnecessary continuous phase transition in a $3+1-D$ fermionic system without gauge fields. Let us consider a $3+1-\mathrm{D}$ topological superconductor (TSC) in the DIII class, namely, a TSC protected by time-reversal symmetry and $\mathcal{T}^{2}=-1$. The interacting fermionic SPT has a $\mathbb{Z}_{16}$ classification $[100,119,120]$ labeled by an integer $\nu$. The low-energy theory for the $\nu=1$ DIII TSC state can be 
represented by a massive Majorana fermion in $3+1-\mathrm{D}$ bulk. Reversing the bulk Majorana mass can tune a trivial superconductor to topological superconductor phase transition. Now, we take 16 copies of the $\nu=1$ DIII TSC states. We can consider the phase transition from the trivial state to the topological state for all copies of the system. Certainly, 16 copies of ${ }^{3} \mathrm{He}-\mathrm{B}$ are adiabatically connected to a trivial phase because the surface has no time-reversal anomaly [100]. However, the transition is not guaranteed to be a single generic transition. Different copies of the system can go through phase transition successively. In order to have a single transition, there must be some flavor-rotation symmetry. The most naive one is an $S O(16)$ symmetry, which rotates the 16 copies of TSC's. This symmetry, together with $Z_{2}^{T}$ symmetry, allows only one Majorana mass term. The bulk low-energy theory is

$$
\mathcal{L}_{\mathrm{TSC}}^{\times 16}=\sum_{i=1}^{16} \bar{\chi}_{i}\left(i \gamma_{\mu} \partial_{\mu}-m\right) \chi_{i}+\cdots
$$

Therefore, there is a generic continuous phase transition when we tune the mass from negative to positive. However, in this situation, the two sides of the phase transition are different topological phases protected by the $S O(16)$ symmetry. In particular, on one side, $m<0$, we can regularize the system to be in the trivial phase, where we have a trivial response theory for the $S O(16)$ background gauge field. On the other side, $m>0$, the response theory of the background $S O(16)$ gauge field has a $\Theta$ term, with $\Theta=\pi$, which indicates that the system is a SPT state protected by the $S O(16)$ symmetry. Since the two sides are distinct topological phases of the $S O(16)$ symmetry, there will always be a phase transition separating them. This case seems to be a disappointing one. Nonetheless, a slight modification of the global symmetry gives us an example of an unnecessary continuous phase transition.

Consider breaking the flavor symmetry from $S O(16)$ to $S O(2) \times S O(7)$ symmetry. The symmetry action on the fermions can be understood in the following way. Let us pack the 16 Majorana fields into a $2 \times 8$ matrix. The $S O(2)$ and $S O(7)$ symmetries are implemented by the left and right multiplication by orthogonal matrices. Here, the right multiplications are in the eight-dimensional spinor representation of the $S O(7)$ group. This symmetry only allows the $\bar{\chi} \chi$ mass. To see this case, we can write down the general form of the Lorentz and time-reversal symmetric mass term $\bar{\chi}_{i} S_{i j} \chi$, where $S_{i j}$ is a real symmetric matrix in flavor space. Note that $S$ can, in general, be decomposed into two classes: $S \sim S_{2} \otimes S_{8}$ or $S \sim A_{2} \otimes A_{8}$, where $S_{2}$ and $S_{8}$ denote real symmetric matrices of dimension 2 and 8 , and $A_{2}$ and $A_{8}$ are antisymmetric matrices of dimension 2 and 8. The $S O(2)$ generators are $A_{2} \otimes \mathcal{I}_{8}$ and $S O(7) \in \mathcal{I}_{2} \otimes A_{8}$. We can explicitly check that the only matrix that commutes with all the $S O(2)$ and $S O(7)$ generators is $\mathcal{I}_{2} \otimes \mathcal{I}_{8}$, which is the identity. Therefore, the $\bar{\chi} \chi$ term is the only allowed mass term. This means that, with $S O(2) \times S O(7)$ symmetry, there is still a generic phase transition as we tune the mass from negative to positive. Since the phase transition is described by free fermions, it is stable against small interactions.

Next, we show that in the $S O(2) \times S O(7)$ case, the $m<0$ and $m>0$ phases are actually the same phase. We argue this through the surface state of the system. At the free fermion level, the natural 2+1-D surface state of the $m>0$ phase has 16 gapless Majorana fermions. We argue that the surface state can become symmetrically gapped out by interaction, which indicates that the bulk state is in the same class as the trivial state. First, let us organize the 16 Majorana fermions into 8 Dirac fermions:

$$
H=\sum_{i=1}^{8} \psi_{i}^{\dagger}\left(p_{x} \sigma_{x}+p_{y} \sigma_{z}\right) \psi_{i} .
$$

The $S O(2)$ or the $U(1)$ is now the phase rotation of the $\psi$ fermion. The time-reversal action is $\mathcal{T}: \psi \rightarrow i \sigma^{y} \psi^{\dagger}$. The $\psi_{i}$ 's also form the spinor representation of the $S O(7)$ symmetry. Then, we introduce a spin singlet pairing in the theory, which completely gaps out the surface state.

$$
H_{\text {pairing }}=\sum_{i=1}^{8} \Delta \psi_{i}^{T} i \sigma^{y} \psi_{i}+\text { H.c. }
$$

This pairing obviously preserves the $S O(7)$ symmetry. It breaks both $U(1)$ and the time reversal $\mathcal{T}$. However, it preserves another anti-unitary symmetry $\tilde{\mathcal{T}}=\mathcal{T} U(\pi / 2)$ [100]. The next step is to quantum fluctuate the pairing order parameter to restore the symmetries. This fluctuation can be done by condensing the $2 \pi$ vortices of the pairing order parameter. There are two key requirements for getting a symmetric gapped surface state after the condensation. First of all, in order to restore both $U(1)$ and $\mathcal{T}$, the condensation has to preserve the $\tilde{\mathcal{T}}$ symmetry. Second, the vortices must carry a gapped spectrum. These conditions need special care because the vortex core of the system carries Majorana zero modes[121]. For our system, in a $2 \pi$ vortex ( $\pi$ flux for fermions), there will be eight Majorana zero modes, $\chi_{i}, i=1, \ldots, 8$. Their $\tilde{\mathcal{T}}$ transformation is $\tilde{\mathcal{T}}: \chi_{i} \rightarrow \chi_{i}$ because the $\tilde{\mathcal{T}}$ does not change the vortex background. This time-reversal symmetry will forbid us to gap out the zero modes by a fermion bilinear term. However, it is well known that an $S O(7)$-invariant fourfermion interaction term, which is the so-called FidkowskiKitaev interaction [122], can give rise to a gapped spectrum for eight Majorana modes. With this interaction, we can condense the $2 \pi$ vortices and get a symmetric gapped surface state. This result indicates that the bulk state is topologically trivial. 
The phase diagram of the system is demonstrated in Fig. 6. The $m$ term precisely corresponds to the free fermion mass and $U_{\text {int }}$ to the Fidkowski-Kitaev interaction. The free fermion phase transition in $3+1-\mathrm{D}$ is stable against small interactions. In the limit of large interactions, we can first diagonalize the on-site interaction and treat the kinetic term as a perturbation. In the large interaction limit, the system is essentially a trivial insulator with a tensor-product wave function. Therefore, the phase transition can be avoided by going through the strongly interacting part of the phase diagram.

\section{FERMIONIC DECONFINED CRITICAL POINTS IN 3 + 1-D}

In this section and the following ones, we study quantum critical points that can be formulated as $3+1$-D $S U(2)$ gauge theory coupled to $N_{f}$ flavors of massless adjoint Dirac fermions, denoted as $S U(2)+N_{f}^{A}$ theory. Based on the perturbative calculation, for $N_{f}^{A}>2$, the theory is free in the infrared limit. Numerically, the $N_{f}^{A}=2$ theory is inside the conformal window [90]. There are also numerical indications that the $N_{f}^{A}=1$ theory is conformal in the IR [89]. In this section, we study in detail the IR-free $S U(2)$ gauge theories with $N_{f}^{A}=3$ massless adjoint Dirac fermions and interpret them as quantum critical points between fermionic SPT states. Since the gauge theory is free in the IR, we can make many precise statements. Generalizations to arbitrary $N_{f}$ and possibly other gauge groups are discussed in the next section and in Appendixes G-I.

\section{A. $S U(2)$ gauge theory with $N_{f}=3$ adjoint fermions}

We consider a quantum critical point that can be described by $3+1-\mathrm{D} S U(2)$ gauge theory with three flavors of adjoint Dirac fermions. The case is very similar for all the odd $N_{f}^{A}>3$. We label the fermions by $\psi_{i}^{a}$, where $a=1,2,3$ is the $S U(2)$ gauge index, and $i=1,2,3$ is the flavor index. A key difference from the fundamental fermion case is that there are gauge-singlet fermion operators (the baryons) such as $\epsilon_{a b c} \psi^{a} \psi^{b} \psi^{c}$ and $\epsilon_{a b c} \psi^{a \dagger} \psi^{b} \psi^{c}$ (Lorentz indices are suppressed here). Indeed, all local operators of the theory carry quantum numbers that can be built up as composites of these baryons. Therefore, the $S U(2)$ gauge theory with adjoint fermion fields describes a critical theory in intrinsic fermionic systems.

The Lagrangian for the $N_{f}^{A}=3$ theory reads

$\mathcal{L}_{\text {adj }}^{N_{f}^{A}=3}=\sum_{j=1}^{3} i \bar{\psi}_{j} \gamma_{\mu}\left(\partial_{\mu}-a_{\mu}^{i} T^{i}\right) \psi_{j}-m \bar{\psi}_{j} \psi_{j}+\cdots$,

where $\left\{\gamma_{0}, \gamma_{1}, \gamma_{2}, \gamma_{3}\right\}=\left\{\sigma^{12}, i \sigma^{03},-i \sigma^{22},-i \sigma^{01}\right\}, \quad \gamma_{5}=$ $i \gamma_{0} \gamma_{1} \gamma_{2} \gamma_{3}=\sigma^{32}$, and $T^{i}, i=1,2,3$, are the $S U(2)$ generators in the spin-1 representation. The theory has a $Z_{2}^{T}$ symmetry [123] whose transformation on the fermion fields is as follows:

$Z_{2}^{T}: \psi(\mathbf{x}, t) \rightarrow \gamma_{0} \gamma_{5} \psi^{\dagger}(\mathbf{x},-t)=-i \sigma^{20} \psi^{\dagger}(\mathbf{x},-t), \quad i \rightarrow-i$.

Following the method in previous sections, we can construct the adjoint $S U(2)$ theory from 18 Majorana fermions and then gauge the diagonal $S O(3)$ part of the total $S O(18)$ flavor symmetry. Since $S O(18) \supset S O(3) \times S O(6)$, the global symmetry after gauging is $\mathcal{G}=S O(6) \times Z_{2}^{T}$ [124]. The Dirac mass in Eq. (62) is the only mass term allowed by the global symmetry.

The theory in Eq. (62) also has a global 1 -form $Z_{2}$ center symmetry [125] because we did not include any matter field in the $S U(2)$ fundamental representation. The physical manifestation of the 1-form symmetry is that the Hilbert space of the system contains unbreakable spin- $1 / 2$ electric flux loops. However, if we are to view the gauge theory as emerging from a UV system of gauge-invariant fermions (defined, perhaps, on a lattice), the 1-form symmetry can only be an infrared emergent symmetry. Therefore, we should allow for explicit breaking of the 1-form symmetry in the UV. To that end, we introduce a massive spin- $1 / 2$ particle into our theory, which we call the spectator field. The spectator field allows the spin- $1 / 2$ electric flux loops to break. We emphasize that, from the point of view adopted in this paper, the theory in Eq. (62) is not complete yet because we did not specify the properties of the spin- $1 / 2$ spectator fields under the global symmetry $\mathcal{G}$. To have a complete theory, we need to specify the symmetry charges of the spectator field under the 0 -form global symmetry $\mathcal{G}$. (This method is, in some sense, equivalent to defining the symmetry properties of the spin- $1 / 2$ electric flux lines.) Perhaps surprisingly, the symmetry charges of the massive spectator field crucially determine the nature of the $m \neq 0$ phases of this theory, although they do not participate in the low-energy physics at all. We explain this phenomenon in detail later. For now, let us restrict our attention only to the 0 -form global symmetry of the system, which is $\mathcal{G}=S O(6) \times Z_{2}^{T}$.

The theory in Eq. (62) at the massless point is a free theory in the infrared. The fermion mass is a relevant perturbation that will drive the system to the infinite-mass fixed point. Thus, the massless theory describes a continuous quantum phase transition between the $m<0$ and $m>0$ phases. The schematic renormalization group flow of the fermion mass and gauge coupling is similar to that in Fig. 3. Let us identify the phases with large negative or positive fermion masses. For large fermion mass, we can integrate out the fermions first. We choose a UV regularization such that, in the $m<0$ phase, the $S U(2) \Theta$ term generated by integrating out the massive fermions is zero. The $S U(2)$ gauge theory is confined at low energy, and the resulting state is a trivial gapped state. For the large $m>0$ phase, 
one can show that the $\Theta$ angle is $12 \pi$ for the $S U(2)$ gauge fields [126]. This case is also trivial because of the $2 \pi$ periodicity of the $\Theta$ angle, and the $S U(2)$ gauge theory is again in a confined phase. In particular, both confined phases are believed to be in a short-range-entangled ground state. For both signs of the mass, in the large mass limit, we expect a gapped and nondegenerate ground state with no symmetry breaking. They must fall into the classification of the fermionic SPT states with $S O(6) \times Z_{2}^{T}$ symmetry. Since this symmetry class is not usually considered in the literatures, let us first discuss the interacting classification of such fermionic SPT phases.

The classification of fermion SPTs for this symmetry in $3+1-\mathrm{D}$ is $\mathbb{Z}_{8} \times \mathbb{Z}_{2}$, which can be labeled by two indices $n \in \mathbb{Z}_{8}$ and $\eta \in \mathbb{Z}_{2}$. The $\mathbb{Z}_{2}$ part comes from the pure $Z_{2}^{T}$ SPT labeled [127] by efmf. The $\mathbb{Z}_{8}$ part is the reduced classification from the free fermion SPT with the same symmetry. Note that at the free fermion level, SPTs with this symmetry have a $\mathbb{Z}$ classification, which we label by the same index $n$. The root $n=1$ state of the free fermion SPT with $S O(6) \times Z_{2}^{T}$ symmetry can be viewed as six copies of the topological superconductor with $Z_{2}^{T}$ symmetry, namely, the DIII class. The six copies form a vector representation under $S O(6)$. At the surface, the $n=1$ state has (within free fermion theory) six massless Majorana fermions. For general $n$, there will correspondingly be $6 n$ massless Majorana fermions at the surface. With interactions, we need to consider whether, for some special $n$, the surface is anomaly-free. The anomaly on the surface has two parts: (1) a pure time-reversal anomaly and (2) a mixed anomaly between the $S O(6)$ and $Z_{2}^{T}$, which is sometimes called the (generalized) parity anomaly in the literature. The pure time-reversal anomaly is $\mathbb{Z}_{16}$-fold. Physically, this means 16 copies of Majorana fermions in 2+1-D are timereversal anomaly-free. Therefore, at least eight copies of the root states are needed to cancel the surface time-reversal anomaly. The mixed anomaly between $S O(6)$ and $Z_{2}^{T}$ or the parity anomaly is fourfold [128].

The physical diagnosis for this mixed anomaly is the quantum number of the background $S O(6)$ monopole. One can show that, for four copies of the root state, the monopole of the background $S O(6)$ gauge field is a trivial boson. Therefore, the surface of the $n=8$ state is totally anomaly-free. Hence, with interactions, the free fermion SPT classification collapses to $\mathbb{Z}_{8}$. In addition, the $n=4$ state corresponds to the $e \operatorname{Tm} T$ state $[42,127]$. For $n=4$, there is no parity anomaly involving $S O(6)$ and $Z_{2}^{T}$. The surface anomaly purely comes from the time-reversal anomaly. For $n=4$, the surface theory has $4 \times 6=24$ Majorana fermions. Since the time-reversal anomaly is $\mathbb{Z}_{16}$ periodic, the surface corresponds to the surface of the $\nu=$ $24 \sim 8$ state in the DIII class, which is precisely equivalent to the $e T m T$ anomalous surface.

Let us always assume that the $m<0$ phase is the trivial state $(n=0, \eta=0)$, which can be achieved by certain UV regularization. The question is which $(n, \eta)$ the $m>0$ phase falls into. To answer this question, we derive the topological response to a background $S O(6)$ gauge field with the same method as used before, namely, gauging the total $S O(18)$ group and restricting the gauge configurations to its subgroups. The topological action for the $m>0$ phase (on an arbitrary, closed oriented space-time manifold) is

$$
\begin{aligned}
\mathcal{S}_{\text {topo }} & =i \frac{\pi}{2}\left(p_{1}\left(A^{S O(18)}\right)-\frac{9}{4} \sigma\right) \\
& =i \frac{\pi}{2}\left(3 p_{1}\left(A^{S O(6)}\right)+6 p_{1}\left(a^{S O(3)}\right)-\frac{9}{4} \sigma\right) \\
& =i 3 \pi\left(\frac{1}{2} p_{1}\left(A^{S O(6)}\right)-\frac{3}{8} \sigma\right)+i \pi p_{1}\left(a^{S O(3)}\right) \\
& =i 3 \pi\left(S_{\theta}^{S O(6)}-\frac{3}{8} \sigma\right)+i \pi \mathcal{P}\left(w_{2}^{S O(3)}\right),
\end{aligned}
$$

where $S_{\theta}^{S O(6)}$ is the usual $\Theta$ term for the $S O(6)$ background gauge field, and the combination $\left(S_{\theta}^{S O(6)}-3 \sigma / 8\right)$ is always an integer. The response theory until now indicates that the $m>0$ phase is a nontrivial topological state. However, it is not enough to exactly determine the topological index of the state. In particular, we cannot tell whether the system belongs to the $n=3$ state or the $n=7 \sim-1$ state. The difference between the two is the $n=4$ state or the $e T m T$ state, whose partition function is always trivial on an orientable manifold. It turns out that to settle this difference, we have to consider the symmetry properties of the spectator field. We shall see that different symmetry properties of the spectator field lead to different topological phases on the $m>0$ side.

To demonstrate the importance of the spectator field, we consider the following two different choices of spectators. There are other ways to choose spectator fields. We leave them to future studies. From the discussions below, we shall see that the symmetry properties of the spectator field crucially determine the nature of the $m>0$ phase.

\section{B. Band-theory-forbidden phase transition between band-theory-allowed insulators}

The simplest choice of the spectator is a bosonic particle that is neutral under all global symmetries, namely, an $S U(2)$ spin- $1 / 2$ boson, which is a scalar under $S O(6)$ and has $\mathcal{T}^{2}=1$. We see that this choice of spectator field leads to an interesting type of band-theory-forbidden phase transition between two band-theory-allowed states.

To consistently define this spectator field, we have a constraint on the gauge connections

$$
w_{2}^{S O(3)}=0 \quad \bmod 2 .
$$


This relation must be satisfied on any base manifold $Y_{4}$. Then, the topological response can be simplified as

$$
\mathcal{S}_{\text {topo }}^{A}=i 3 \pi\left(S_{\theta}^{S O(6)}-\frac{3}{8} \sigma\right),
$$

which suggests $n=3$ in the $\mathbb{Z}_{8}$ classification.

To confirm the nature of the topological phase, let us investigate the surface state of the system. The natural surface state of the system is a $\mathrm{QCD}_{3}$ theory with an $S U(2)$ gauge field coupled to three flavors of massless adjoint Dirac fermions [19]. The action for the $2+1-D$ surface theory can be written as follows:

$$
\begin{aligned}
\mathcal{L}_{\text {surf }}= & \sum_{j=1}^{3} i \bar{\psi}_{j} \gamma_{\mu}\left(\partial_{\mu}-i a_{\mu}^{i} T^{i}\right) \psi_{j}+\left|\left(\partial_{\mu}-i a_{\mu}^{i} \frac{\sigma^{i}}{2}\right) z\right|^{2} \\
& +m|z|^{2}+\cdots,
\end{aligned}
$$

where $\left\{\gamma_{0}, \gamma_{1}, \gamma_{2}\right\}=\left\{\sigma_{y},-i \sigma_{z}, i \sigma_{x}\right\}$. Here, we explicitly include the massive spectator field labeled by $z$. The timereversal symmetry and gauge transformations are

$$
\begin{gathered}
Z_{2}^{T}: \psi \rightarrow i \gamma_{0} \psi^{\dagger}, \\
Z_{2}^{T}: z \rightarrow z^{*}, \\
S U(2): \psi^{a} \rightarrow\left(e^{i \theta^{a} T^{a}}\right)_{a b} \psi^{b}, \\
S U(2): z \rightarrow e^{i \theta^{a}\left(\sigma^{a} / 2\right)} z .
\end{gathered}
$$

The surface theory in Eq. (68) is not very illuminating to us because it involves gauge fields. We want to deform the surface theory in a symmetry-preserving manner to a more familiar surface state. Notice that the spectator boson $z$ is only charged under the $S U(2)$ gauge group. Let us condense the $z$ boson, i.e., go into a "Higgs" phase with $\langle z\rangle \neq 0$. This condensate preserves the $S O(6) \times Z_{2}^{T}$ global symmetry. Furthermore, the condensate completely Higgses the $S U(2)$ gauge fields because $z$ carries the fundamental representation of the $S U(2)$ gauge group. There are no residual gauge fields left on the surface. As a result, the $\psi$ fermions become physical fermions. The surface state now consists of 18 physical massless Majorana fermions with $S O(6) \times Z_{2}^{T}$ symmetry. This is precisely the surface of the $n=3$ state in the $S O(6) \times Z_{2}^{T}$ fermionic SPT classification.

This theory implies a very interesting schematic phase diagram, which is shown in Fig. 7. The phase transition between the trivial state and the $n=3$ state in the $S O(6) \times$ $Z_{2}^{T}$ class can occur in two different ways. In the weakly interacting limit, a trivial superconductor can only become an $n=3$ TSC by three successive topological phase transitions. At each step, the topological index can only jump by one, and the low-energy theory is described by six massless Majorana fermions with $S O(6)$ symmetry.

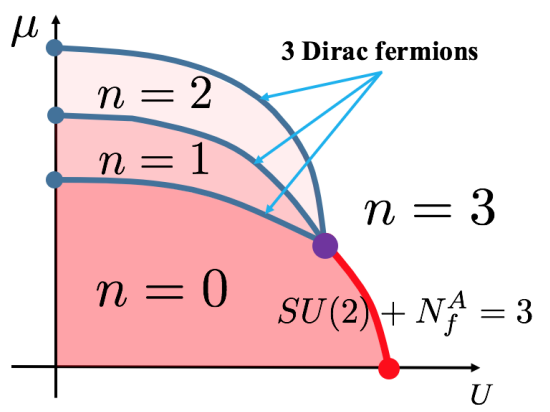

FIG. 7. A schematic phase diagram. The $\mu$ axis represents some parameter that can tune the transition from $n=0$ to $n=3 \mathrm{SPT}$ states with $S O(6) \times Z_{2}^{T}$ symmetry in the free fermion limit. Generically, the free fermion transition will split into three transitions, each of which is described by three massless Dirac fermions (or six Majorana fermions) in the bulk. However, there can be a single transition in the strongly interacting region. The critical theory for the single transition is the $S U(2)+N_{f}^{A}=3$ gauge theory, which is infrared-free. The phase diagram shows one natural postulation about the intermediate coupling regime of the phase diagram. The three weakly coupling phase transitions may merge into a single multicritical point, which could be described by an $S U(2)+N_{f}^{A}=3 \mathrm{QCD}_{4}$-Higgs transition.

However, the $S U(2)+N_{f}^{A}=3$ formulation suggests another very striking possibility that, in the strong interaction region, it is possible to go between the trivial topological state and the $n=3$ state through a single, generic, second-order transition. It is a quantum phase transition between two band insulators, which is forbidden by band theory. In Appendix D, we give a very simple example of this phenomenon that does not involve emergent gauge fields.

These two possibilities for the phase transition may merge at a multicritical point somewhere in the phase diagram. One possible theory for the multicritical point is the Higgs transition of the bosonic spin- $1 / 2$ spectator in the $3+1-\mathrm{D}$ bulk. Once the spectator is condensed in the bulk, the $S U(2)$ gauge fields are completely Higgsed out, and each flavor of the adjoint fermions becomes three physical fermions with topological band structure. Let us label the physical fermions by $c_{j}^{a}, a, j=1,2,3$. They can be expressed by the gauged fermion $\psi$ and spectator field $z$. More explicitly, the resultant physical fermions have the following form:

$$
\begin{aligned}
c_{j}^{1} & \sim \psi_{j} \cdot\left(z^{\dagger} \sigma z\right), \\
c_{j}^{2} & \sim \psi_{j} \cdot \operatorname{Re}\left(z^{t} i \sigma^{y} \sigma z\right), \\
c_{j}^{3} & \sim \psi_{j} \cdot \operatorname{Im}\left(z^{t} i \sigma^{y} \sigma z\right) .
\end{aligned}
$$

The $c$ fermions are gauge-invariant operators. It can be easily checked that the $c$ fermions share the same symmetry transformation as the $\psi$ fermions. The three successive 
phase transitions can be viewed as the mass inversion transition for each flavor of the $c$ fermion.

It is interesting to ask what happens if we first take the mass of the fundamental spectator scalar to infinity. Then, the gauge theory has the $Z_{2} 1$-form symmetry associated with the spin- $1 / 2$ electric flux loops. This symmetry is spontaneously broken in the free theory that emerges at the massless point. Upon perturbing with a fermion mass, the gauge theory enters a confined phase. Then, the electric flux loops acquire a line tension, and the $\left(Z_{2}\right)_{1}$ symmetry is restored. The spin- $1 / 2$ electric loops are, however, decoupled from other excitations. If we now reintroduce the fundamental scalars to explicitly break the $\left(Z_{2}\right)_{1}$ symmetry, in either phase, the loops can break, but the sole effect on the phase is to determine the SPT character. At the massless critical point, the explicit breaking of the $\left(Z_{2}\right)_{1}$ has no effect on low-energy critical properties of the fermions. The spectator scalars will be deconfined at the critical point and gapped away from it.

\section{Multiple universality classes in fermionic phase transitions}

Another choice of the massive spectator field is a (gauge) spin-1/2 bosonic particle, which is an $S O(6)$ scalar but a Kramers doublet under time reversal, namely, $Q_{S O(6)}=0$ and $\mathcal{T}^{2}=-1$. This choice of spectator field implies the following constraint on the gauge connection:

$$
w_{2}^{S O(3)}=w_{1}^{T Y} \cup w_{1}^{T Y} \bmod 2 .
$$

Equations (66) and (74) are the fundamental differences between the two theories. The difference only arises when we consider the gauge bundle on nonorientable [129] manifolds such as $\mathbb{R P}^{4}$. This relation implies that, on an orientable manifold, $w_{2}^{S O(3)}=0 \bmod 2$, meaning that the $S O$ (3) connection can always be lifted to an $S U(2)$ connection. In this case, we get the same topological response theory, Eq. (7), on an orientable manifold:

$$
\mathcal{S}_{\text {topo }}^{B}=i 3 \pi\left(S_{\theta}^{S O(6)}-\frac{3}{8} \sigma\right) .
$$

It appears that this state also corresponds to the $n=3$ state. However, it is known that the $e T m T$ state, which corresponds to the $n=4$ state $[100,130]$, is only visible in the partition function on a nonorientable manifold [128,131133]. Therefore, the topological response on an orientable manifold cannot tell us precisely what topological phase the $m>0$ state belongs to. In the following, we instead use similar physical surface arguments as in the previous section to determine the topological index of this system.

To determine the nature of the $m>0$ phase, we again look at the boundary state. The surface theory has the same form as the $\mathrm{QCD}_{3}$ theory written in Eq. (68), while the only difference is the time-reversal symmetry transformation on $z$ [134],

$$
Z_{2}^{T}: z \rightarrow i \sigma_{y} z, \quad i \rightarrow-i .
$$

In this situation, it appears that condensing the bosonic spectator field would break the time-reversal symmetry. However, the condensate actually preserves the physical time-reversal symmetry. The reason for this is that $z$ is not gauge neutral, and the time-reversal transformation on $z$ can always be combined with an $S U(2)$ gauge rotation. Physical time-reversal symmetry is preserved so long as such a combination of the time-reversal action in Eq. (76) and an $S U(2)$ gauge rotation can keep the action invariant. Explicitly, we consider a gauge-equivalent time-reversal transformation $\tilde{Z}_{2}^{T}$ [135]:

$$
\tilde{Z}_{2}^{T}: z \rightarrow i \sigma^{y} e^{-i\left[\left(\pi \sigma^{y}\right) / 2\right]} z=z, \quad i \rightarrow-i .
$$

The boson is a Kramer's singlet for this time-reversal symmetry transformation [136]. Notice that this timereversal transformation also has a different action on the gauged fermion fields by an additional gauge rotation (here, we suppress the flavor index because all the operations are identical for the three flavors),

$$
\tilde{Z}_{2}^{T}: \psi^{a} \rightarrow i \gamma_{0}\left(e^{-i \pi T^{y}}\right)_{a b} \psi^{b \dagger}, \quad i \rightarrow-i .
$$

In component form,

$$
\begin{aligned}
\tilde{Z}_{2}^{T}: \psi^{1,3} & \rightarrow-i \gamma_{0} \psi^{1,3 \dagger}, \\
\psi^{2} & \rightarrow i \gamma_{0} \psi^{2 \dagger} .
\end{aligned}
$$

Now, let us condense the spectator boson with $\langle\operatorname{Im}(z)\rangle=0$ and $\langle\operatorname{Re}(z)\rangle \neq 0$. This condensate completely Higgses the $S U(2)$ gauge theory while preserving the $S O(6) \times \tilde{Z}_{2}^{T}$ symmetry. The three adjoint fermions become nine physical Dirac fermions. However, we need to be careful about their time-reversal transformation in order to determine the topological index. In particular, the relative sign of the time-reversal transformation of the surface Dirac fermions plays an important role here. In our convention, the Dirac fermion with the "+" transformation, namely, $\psi \rightarrow+i \gamma_{0} \psi^{\dagger}$, contributes $n=+1$ to the topological index for the bulk. Correspondingly, the "-" transformation will contribute $n=-1$ [83]. Based on the transformation in Eq. (79), the surface state corresponds to the $n=-1+1-$ $1=-1$ state in the $\mathbb{Z}_{8}$ classification.

From the above physical arguments, we see that the spectator field plays an important role in defining the global structure of the gauge fields and in determining the nearby topological phase, although it is massive and never appears at low energy near the critical point. To our knowledge, this is not a widely appreciated phenomenon. However, it is not uncommon. We include an example of this phenomenon in a 2+1-D bosonic Mott insulator to a time-reversalsymmetry-enriched $Z_{2}$ spin liquid transition in Appendix E.

This system provides a clear example of multiple universality classes in fermionic systems. The transition 
between the $n=0$ state and the $n=-1$ state can happen within band theory. The critical theory is described by three massless Dirac fermions in the bulk with the $S O(6) \times Z_{2}^{T}$ symmetry. The $S U(2)+N_{f}^{A}=3$ theory gives another phase transition theory between the $n=0$ and $n=-1$ states. We know in the IR that this theory contains just free $S U(2)$ gluons and nine Dirac fermions, which is clearly different from the critical theory in the free fermion limit. These two theories not only differ by their matter contents but also by the emergent symmetries at the critical point. In particular, the gauge theory has an emergent $Z_{2} 1$-form symmetry, which is spontaneously broken in the IR.

The theory discussed in this section is readily generalizable to all odd $N_{f}^{A}>3$. With general $N_{f}^{A}$, the global symmetry of the system is $S O\left(2 N_{f}^{A}\right) \times Z_{2}^{T}$. The interacting fermionic SPT classification for this class is again $\mathbb{Z}_{8} \times \mathbb{Z}_{2}$. With a Kramers singlet bosonic spectator field $[S U(2)$ gauge spin-1/2 and $S O\left(2 N_{f}^{A}\right)$ scalar], the massless $S U(2)+N_{f}^{A}$ theory describes a phase transition between $n=0$ and $n=3$ SPT states in this symmetry class, which provides new examples of band-theory-forbidden continuous phase transitions between band-theory-allowed states. For a Kramers doublet bosonic spectator, the massless $S U(2)+N_{f}^{A}$ theory is a theory of a continuous phase transition between the $n=0$ and $n=-1$ SPT states. Since $N_{f}^{A}$ is large enough, this theory contains only free gluons and free fermions in the IR. Another route for such a phase transition is a free fermion phase transition that is characterized by free massless Dirac fermions in the $3+1-\mathrm{D}$ bulk. These two critical theories are obviously distinct from each other. They demonstrate another example of multiple universality classes for phase transitions in fermionic systems.

The $S U(2)$ gauge theories with even $N_{f}^{A}$ flavors of massless adjoint Dirac fermions can also be interpreted as quantum critical points between fermionic SPT states. The phenomenology of the even series is slightly different from the odd series. In particular, the topological phase on the $m>0$ side of the phase diagram does not depend on the choice of the spectator field. We present the example of $N_{f}^{A}=2$ in Appendix G. It is straightforward to generalize the theory to larger, even $N_{f}^{A}$. We also provide generalizations to the $S U(2)+N_{f}^{A}$ theories with half-integer $N_{f}^{A}$, as well as a generalization to $S U(4)$ gauge theory with $N_{f}^{A}=1$ flavor of adjoint fermion in the Appendixes $\mathrm{H}$ and $\mathrm{I}$.

\section{THE $S U(2)$ GAUGE THEORY WITH ONE-FLAVOR ADJOINT DIRAC FERMION}

The $S U(2)+N_{f}^{A}=1$ theory is a special case for the odd $N_{f}^{A}$ series. The global symmetry in this case is $S O(2) \times$ $Z_{2}^{T} \simeq U(1) \times Z_{2}^{T}$, which is the symmetry of the topological superconductor in the AIII class. Since this theory is a strongly interacting gauge theory in the IR, its low-energy fate is much more subtle than previous examples. We discuss this theory in detail in the following sections. We notice there is some numerical evidence that this theory is conformal in the IR [89]. We explore its interpretation as a quantum critical point.

Note that the fermion mass is a relevant perturbation for the massless $S U(2)+N_{f}^{A}=1$ theory [89]. However, the massless $S U(2)+N_{f}^{A}=1$ theory is strongly coupled in the gauge-theory description. A priori, we do not know whether an infinitesimal mass $m$ perturbation will lead the RG flow to the infinite-mass fixed point. If small mass does lead to a flow to the infinite-mass limit, we will have a direct secondorder phase transition between the two gapped phases. If this is not the case, there may be an intermediate phase in the small-mass limit. In this section, we only discuss the properties of the system with large fermion mass, and we determine the distinct gapped phases. Inspired by this understanding, in Sec. VI, we describe a possible IR theory of the massless $S U(2)+N_{f}^{A}=1$ theory. We see that, within this proposed IR theory, there are indeed intermediate phases for small $m$ that differ from the large- $m$ phases by the presence of an extra topological ordered state.

\section{A. Global symmetry and topological response}

As mentioned in the previous section, the $S U(2)$ gauge theory with adjoint fermion fields describes a critical theory in intrinsically fermionic systems. The Lagrangian for the $N_{f}^{A}=1$ theory reads

$$
\mathcal{L}_{\text {adj }}^{N_{f}^{A}=1}=i \bar{\psi} \gamma_{\mu}\left(\partial_{\mu}-a_{\mu}^{i} T^{i}\right) \psi-m \bar{\psi} \psi+\cdots
$$

The theory has $U(1) \times Z_{2}^{T}$ global symmetry whose transformations on the fermion fields are as follows:

$$
\begin{gathered}
U(1): \psi \rightarrow e^{i \theta} \psi, \\
Z_{2}^{T}: \psi(\mathbf{x}, t) \rightarrow \gamma_{0} \gamma_{5} \psi^{\dagger}(\mathbf{x},-t), \quad i \rightarrow-i .
\end{gathered}
$$

Note that $U(1) \times Z_{2}^{T}$ is the symmetry for a topological superconductor in the AIII class in the condensed matter language. The Dirac mass in Eq. (80) is the only mass term allowed by the symmetry. As written, the theory in Eq. (80) also has a global 1-form $Z_{2}$ center symmetry because of the absence of the matter fields in the gauge $S U(2)$ fundamental representation. However, as we emphasized before, this gauge theory is to be viewed as an emergent theory from a UV system of gauge-invariant fermions, where there is no 1-form symmetry. Therefore, we impose explicit breaking of the 1-form symmetry in the UV by introducing a massive spin-1/2 spectator field into our theory. In this section, we only consider the 0 -form global symmetry of the system, which is $\mathcal{G}=U(1) \times Z_{2}^{T}$. 
We want to explore the theory in the large fermion mass limit. We can then analyze the theory by integrating out the fermions first. We choose a UV regularization such that, in the $m<0$ phase, the $S U(2) \Theta$ term is zero. The $S U(2)$ gauge theory is confined at low energy, and the resulting state is a trivial gapped state. For the large $m>0$ phase, one can show that the $\Theta$ angle is $4 \pi$ for the $S U(2)$ gauge fields. This is also trivial because of the $2 \pi$ periodicity, and the $S U(2)$ gauge theory is again in a confined phase. In particular, both confined phases are believed to be in a short-range-entangled ground state.

For both signs of the mass, in the large-mass limit, we expect a gapped and nondegenerate ground state with no symmetry breaking. They must fall into the classification of the AIII TSC in $3+1-D$, which, as we mentioned before, is $\mathbb{Z}_{8} \times \mathbb{Z}_{2}$ once we include interaction effects [100]. We again denote different AIII TI states by two labels $n \in \mathbb{Z}_{8}$ and $\eta \in \mathbb{Z}_{2}$. The $n \neq 0$ states are descendents of the free fermion AIII TSC. The typical $2+1$-D surface state is $n$ flavors of massless Dirac fermions. The $n=4$ state is in the same phase of a bosonic SPT protected by $Z_{2}^{T}$ symmetry, which is usually signified by its surface $Z_{2}$ topological order, the so-called $e \operatorname{Tm} T$ state $[42,51,127]$. The $\eta=1$ state is another $Z_{2}^{T}$ bosonic SPT state, whose surface $Z_{2}$ topological order is the so-called efmf state [42]. Let us always assume that the $m<0$ phase is the trivial state $(n=0, \eta=0)$. We want to determine which $(n, \eta)$ the $m>0$ phase falls into.

We derive the topological response to a background $U(1)$ gauge field with the same method used before. The topological action for the $m>0$ phase (on an arbitrary closed oriented space-time manifold) is

$$
\begin{aligned}
\mathcal{S}_{\text {topo }} & =i \frac{\pi}{2}\left(p_{1}\left(A^{S O(6)}\right)-\frac{3}{4} \sigma\right) \\
& =i \frac{\pi}{2}\left(3 p_{1}\left(A^{S O(2)}\right)+2 p_{1}\left(a^{S O(3)}\right)-\frac{3}{4} \sigma\right) \\
& =i 3 \pi\left(\frac{1}{2} p_{1}\left(A^{S O(2)}\right)-\frac{1}{8} \sigma\right)+i \pi p_{1}\left(a^{S O(3)}\right) \\
& =i 3 \pi\left(S_{\theta}^{U(1)}-\frac{1}{8} \sigma\right)+i \pi \mathcal{P}\left(w_{2}^{S O(3)}\right) .
\end{aligned}
$$

The response theory implies that the $m>0$ phase is a nontrivial topological state. However, as before, we cannot tell precisely which class the system belongs to. There may be a $n=4$ state or an $e T m T$ state attached to the system, whose partition function is always trivial on an orientable manifold. This case can be settled by considering the symmetry properties of the spectator field. Just like in the previous section, we demonstrate that different symmetry properties of the spectator field lead to different topological phases on the $m>0$ side.

\section{B. An alternate argument to identify the massive phases}

It is straightforward to use the argument in the previous section to justify that (1) with a $\mathcal{T}^{2}=1$ charge-neutral spin- $1 / 2$ spectator boson, the $m>0$ phase is the $n=3$, $\eta=0$ state in the AIII class and (2) with the $\mathcal{T}^{2}=-1$ spectator boson, the $m>0$ phase is the $n=-1, \eta=0$ state. We will not repeat this argument again. However, in this section, we provide a different argument to support this result.

We can justify the nature of the gapped phases from another point of view. Let us first consider the structure of the massive phases in the infinitely massive spectator limit. Later, we will reinstate the finite mass of the spectator. We are particularly interested in understanding the anomaly of the surface theory as a window into which SPT phase the bulk system is in. The way to identify the anomaly of the surface state is through the method of anomaly inflow.

Deep in the confined phases, all the $S U(2)$ electric flux lines have line tension. In the infinitely massive spectator limit, the spin-1/2 electric flux lines cannot end in the bulk. In other words, the system has an exact 1-form $Z_{2}$ symmetry. The physical difference between the two spectator choices in this case lies in the properties of the spin- $1 / 2$ electric flux lines. While for the $\mathcal{T}^{2}=1$ case the spin-1/2 line has nothing special associated with it, the $\mathcal{T}^{2}=-1$ case physically corresponds to the situation in which each spin-1/2 line is decorated with a Haldane chain protected by the time-reversal symmetry $[42,137]$.

For our system, the surface anomaly contributions come from both the bulk massive adjoint fermions and the unbreakable spin- $1 / 2$ electric flux loop sector. Here, we want to do a comparison between the $\mathcal{T}^{2}=1$ and $\mathcal{T}^{2}=-1$ spectator cases. Notice that the only physical difference between the two cases is whether or not we decorate the spin- $1 / 2$ loops with a Haldane chain protected by $Z_{2}^{T}$. Since the adjoint fermions are topologically decoupled from the spin-1/2 loops, changing the symmetry properties of these loops should not change the surface anomaly contributed by the bulk adjoint fermions. Therefore, we focus on the differences in the surface anomalies contributed by the loop sector for the $\mathcal{T}^{2}=1$ and $\mathcal{T}^{2}=-1$ spectator cases.

A useful formal approach to identify the surface Hilbert space and anomalies is to couple the system with the background gauge field of the global symmetry. We can study the statistical and symmetry properties of the background symmetry fluxes in the bulk and then use the anomaly inflow argument to identify the surface excitations [138]. Since the anomaly on the surface is a renormalization-group-invariant property of the SPT phase, we can consider the anomalies in the weak coupling or UV limit in which we can do reliable calculations.

The symmetry we are interested in here is the 1-form $Z_{2}$ symmetry. Let us couple the system to a background 2-form gauge field for the 1-form $Z_{2}$ symmetry and consider a background $Z_{2}$ flux. This case corresponds to 


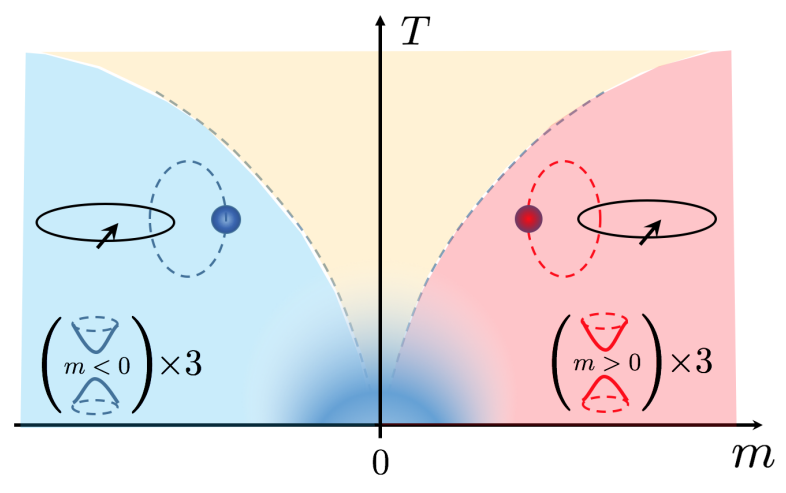

FIG. 8. Deep in the large $m<0$ and $m>0$ phases (the discussion of small fermion mass is postponed to the next section), in the bulk, the $S U(2)$ gauge theory is in a confined phase, where all electric flux lines have line tension. In the infinitely massive spectator limit, the spin- $1 / 2$ electric flux loops cannot break. The system has a global 1 -form $Z_{2}$ symmetry. We can couple the system to a background 2-form $Z_{2}$ gauge field. The $Z_{2}$ flux of the 2-form gauge field physically corresponds to the $S O(3)$ magnetic monopole. It has a nontrivial $\pi$ mutual braiding phase with the spin-1/2 electric flux loops. The statistical and symmetry properties of the $S O(3)$ monopole are different between the $m<0$ phase and the $m>0$ phase because of the topological band structure of the adjoint fermions.

an $S O(3)$ magnetic monopole configuration. The timereversal-symmetry quantum number and statistics of this background monopole will be different between the $m<0$ and $m>0$ phases because the adjoint fermions respond to the $S O(3)$ monopoles. In particular, the fermions in the topological nontrivial band will contribute zero modes to the monopole configurations and potentially change the time-reversal quantum number and the statistics of the monopole. We show that in the $m>0$ phase, the gaugeneutral and global $U(1)$ charge-neutral $S O(3)$ monopole is a Kramers singlet fermion. This statement is independent of the properties of the spin-1/2 Wilson lines. It is an analog of the statistical Witten effect in a bosonic topological insulator with $U(1) \rtimes Z_{2}^{T}$ symmetry [138]. A schematic demonstration of the excitations in the massive phases is shown in Fig. 8.

To demonstrate this, let us put the system on a large sphere and consider a configuration of $2 \pi$ magnetic flux of the $S O$ (3) gauge field coming out of the bulk. For $m<0$, we know that there is no gapless surface state, and the $S O(3)$ monopole carries trivial time-reversal quantum-number and bosonic statistics. However, for $m>0$, the surface theory is a $\mathrm{QCD}_{3}$ with massless adjoint fermions. It is sufficient to calculate the symmetry and statistical properties of the $S O$ (3) monopole in the weak coupling limit-the answers will be unmodified in the strong coupling limit. Let us write down the surface action with a background $S O(3)$ gauge flux along the $z$ direction in color space:

$$
\mathcal{L}_{\text {surf }}=i \bar{\psi} \gamma_{\mu}\left(\partial_{\mu}-i a_{\mu}^{z} T^{z}\right) \psi
$$

where $T^{z}$ is the $S O(3)$ generator along the $z$ direction. We can diagonalize the $T^{z}$ matrix by unitary rotations of the fermions, and it has eigenvalues \pm 1 and 0 . Let us label the three flavors of fermions as $\psi_{+}, \psi_{-}, \psi_{0}\left(\psi_{+} \sim \psi^{x}+i \psi^{y}\right.$, $\left.\psi_{-} \sim \psi^{x}-i \psi^{y}, \psi_{0} \sim \psi^{z}\right)$. Only $\psi_{+}$and $\psi_{-}$are coupled to $a_{\mu}^{z}$, with charge +1 and -1 , respectively. Hence, $\psi_{+}$feels $2 \pi$ flux and $\psi_{-}$feels $-2 \pi$ flux. With rotational symmetry in the color space, every monopole can always be viewed this way. The gauge fluxes in our case are time-reversal invariant since time-reversal symmetry flips the gauge charges instead of the fluxes. From the surface theory in Eq. (85), we know that the $2 \pi$ flux of $a^{z}$ will trap two complex fermion zero modes guaranteed by the index theorem. One zero mode is associated with the $\psi_{+}$fermion, which we label as $f_{+}$. The other one is associated with the $\psi_{-}$fermion, which we label as $f_{-}$. Let us denote the state associated with the flux background with both zero modes empty by $|0\rangle$. There are, in total, four states, which are labeled by $|0\rangle, f_{+}^{\dagger}|0\rangle, f_{-}^{\dagger}|0\rangle, f_{+}^{\dagger} f_{-}^{\dagger}|0\rangle$. Note that $f_{+}$and $f_{-}$ carry opposite gauge charges but the same global $U(1)$ charge. The gauge neutral states from the four states are $|0\rangle$ and $f_{+}^{\dagger} f_{-}^{\dagger}|0\rangle$. However, they carry opposite global $U(1)$ charge of \pm 1 . We can attach the $\psi_{0}$ fermion to the monopole state to compensate the $U(1)$ charge. However, this makes the monopole a fermionic object. Let us label the two states as $\left|M_{1}\right\rangle \sim \psi_{0}^{\dagger}|0\rangle$ and $\left|M_{2}\right\rangle \sim \psi_{0} f_{+}^{\dagger} f_{-}^{\dagger}|0\rangle$. Under time reversal, $Z_{2}^{T}:|0\rangle \rightarrow f_{+}^{\dagger} f_{-}^{\dagger}|0\rangle, \psi_{0} \rightarrow \gamma_{0} \gamma_{5} \psi_{0}^{\dagger}$. The time-reversal transformations on $f_{+}$and $f_{-}$are a bit subtle. After carefully solving the zero-mode wave function in Appendix C, we find $f_{+}^{\dagger} \rightarrow-i f_{+}, f_{-}^{\dagger} \rightarrow i f_{-}$, where the relative minus sign indicates that the fluxes are opposite. With these results, we can work out the time-reversal transformation on the flux as follows:

$$
\begin{aligned}
\left|M_{1}\right\rangle & \sim \psi_{0}^{\dagger}|0\rangle \rightarrow \gamma_{0} \gamma_{5} \psi_{0} f_{+}^{\dagger} f_{-}^{\dagger}|0\rangle \sim \gamma_{0} \gamma_{5}\left|M_{2}\right\rangle, \\
\left|M_{2}\right\rangle & \sim \psi_{0} f_{+}^{\dagger} f_{-}^{\dagger}|0\rangle \rightarrow \gamma_{0} \gamma_{5} \psi_{0}^{\dagger}\left(-i f_{+} i f_{-}\right) f_{+}^{\dagger} f_{-}^{\dagger}|0\rangle \\
& \sim-\gamma_{0} \gamma_{5}\left|M_{1}\right\rangle .
\end{aligned}
$$

Since $\left(\gamma_{0} \gamma_{5}\right)^{2}=-1$, the $S O(3)$ monopole is a Kramer's singlet fermion [139]. Note that this result cannot be altered if we redefine the $Z_{2}^{T}$ transformation by combining with the $U(1)$ phase rotation because the two states are gauge and global charge neutral.

Let consider an interface between the vacuum and our system. Now, imagine a process in which we take a background $S O(3)$ monopole in the vacuum and drag it into our system. This process can be viewed as an instanton event for the $2+1-D$ interface, where the background $S O(3)$ flux changes from 0 to $2 \pi$. The $S O(3)$ monopole is a neutral boson in the vacuum; however, it becomes a neutral fermion in the bulk system. As a result, the instanton event, besides creating a $2 \pi$ background flux on the surface, must also nucleate a neutral fermion excitation, labeled by $f$, in order to conserve the fermion parity of the whole system. 
Therefore, the surface must have a neutral fermion excitation.

Now, let us introduce a finite-mass spin- $1 / 2$ spectator boson on the surface, which can be viewed as the end point of the spin- $1 / 2$ electric flux line on the boundary. We label it by $e$. In the weak coupling limit, it is a deconfined particle on the surface. We need to determine the braiding statistics between $e$ and $f$. The instanton event we described above is a local process on the surface. The locality implies that, if we adiabatically drag the spectator boson $e$ around the location of the instanton event, there should be no difference in the accumulated Berry phase before and after the instanton event. As a result, the braiding phase between the spectator and the neutral fermion $f$ must cancel that between the spectator and the $2 \pi$ background flux. Since the spin- $1 / 2$ spectator can be viewed as the half-charge under $S O(3)$, the braiding phase between the spectator and the $2 \pi$ flux is $\pi$. Therefore, $e$ and $f$ have a mutual $\pi$ braiding phase, and they form a $Z_{2}$ topological order on the surface. Now, let us consider the time-reversal properties of the $Z_{2}$ topological order. For the first case with the $\mathcal{T}^{2}=1$ spectator, we have a vanilla $Z_{2}$ topological order, which is not anomalous. For the other case with the $\mathcal{T}^{2}=-1$ spectator, since $f$ is a Kramers singlet, the bound state $m \sim$ ef is also a Kramers doublet boson. The $Z_{2}$ topological order is the so-called $e T m T$ state, which carries a timereversal anomaly of the $n=4$ state in the AIII class.

We can also include the spin-1/2 matter and break the 1 -form $Z_{2}$ symmetry in the bulk. Dynamically, the $S U(2)$ gauge theory will be in a confined phase for large fermion mass, which means all electric flux lines have finite line tension. With 1-form $Z_{2}$ symmetry, in the confined phase, the system has unbreakable, tensionful, spin- $1 / 2$, electric flux loops. With finite-mass spectators, these loops will break dynamically in the bulk, and the system is in an ordinary confined phase. However, since the time-reversal anomaly on the surface does not involve the 1-form symmetry, it will survive even with a finite spectator mass.

\section{POSSIBLE 3 + 1-D DUALITY}

The $S U(2)+N_{f}^{A}=1$ theory with a $\mathcal{T}^{2}=-1$ spectator field potentially provides a continuous phase transition theory between the $n=0$ and $n=-1$ states in the AIII class. The same phase transition can also happen in a free fermion setting, which is described by a free massless Dirac fermion. There are several possible scenarios about the relationship between the strongly coupled gauge theory and the single Dirac fermion theory. For example, a simple possibility is that the low-energy theory of $S U(2)^{*}+N_{f}^{A}=$ 1 [140] theory is a completely different critical theory from the single Dirac fermion [141]. Perhaps the most exciting scenario is that the $S U(2)^{*}+N_{f}^{A}=1$ theory in the IR is strictly dual to a single Dirac fermion as shown schematically in Fig. 9. Unfortunately, we argue that this scenario is

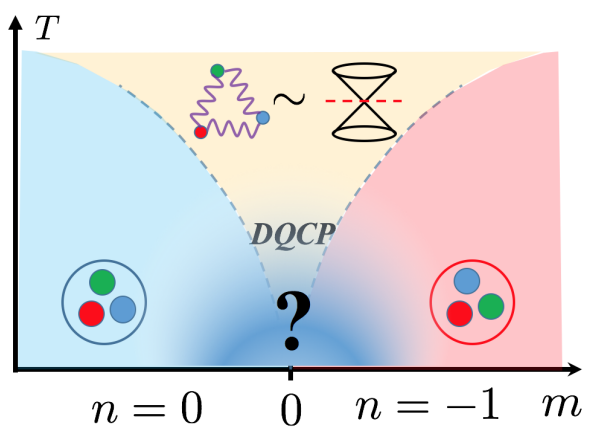

FIG. 9. The transition between the $n=0$ state and the $n=-1$ state can happen in two ways. One way is a free fermion transition with a single gapless Dirac fermion as the critical theory. The other way is through a strongly coupled non-Abelian gauge theory, which we label $S U(2)^{*}+N_{f}^{A}=1$. A very exciting possibility is that the two $3+1-\mathrm{D}$ conformal field theories are dual to each other in the infrared limit. Unfortunately, this is not likely the case. We argue that a possible IR theory is a single Dirac fermion plus a topological field theory.

very unlikely. Instead, a candidate low-energy theory of the $S U(2)^{*}+N_{f}^{A}=1$ theory can be very close to a single Dirac fermion. In particular, we suggest a possible IR theory that contains a single, free, Dirac fermion plus a decoupled gapped topological sector. For energy lower than the gap of the topological order, the theory is described purely by a free Dirac fermion.

An important consistency check on any proposed IR theory is anomaly matching with the UV theory. Our UV theory in the $m=0$ limit has emergent global symmetries that are anomalous. Matching the emergent symmetries and their anomalies between IR and UV provides nontrivial constraints. In particular, our theory in the infinitespectator-mass limit is closely related to the celebrated Seiberg-Witten theory $[59,142]$ whose global symmetry and anomaly structure are well understood in the highenergy literature. Exploiting this idea, Ref. [59] recently provided a very nice discussion of the various anomalies of the $S U(2)$ gauge theory with a single, massless, adjoint Dirac fermion. The exact 1-form $\left(Z_{2}\right)_{1}$ symmetry of this theory was shown to have mixed anomalies with the emergent global symmetry and geometry [59], which put more constraints on the possible low-energy theories. Therefore, we start our discussion from the infinitely massive spectator limit and later reinstate the finite mass to the spectator field. We first identify the emergent 0 -form global symmetry and the anomalies in the $S U(2)+N_{f}^{A}=1$ theory. We see that the 0 -form emergent symmetry and anomaly can indeed be matched by a single Dirac fermion theory. However, the single Dirac fermion does not have the $Z_{2} 1$-form symmetry and hence cannot match the UV anomalies associated with it. This result indicates that the low-energy theory must contain either additional gapless or gapped topological d.o.f. that could compensate the 
anomalies associated with the 1-form symmetry. Reference [59] obtained such a candidate IR theory consisting of a single Dirac fermion plus a decoupled $U(1)$ gauge theory in the Coulomb phase through supersymmetry-breaking deformations from the Seiberg-Witten theory. We propose a different candidate theory, which has a single Dirac fermion plus a decoupled topological order [143]. The possibility of a topologically ordered state was also briefly mentioned in Ref. [59].

\section{A. The IR Dirac fermion}

Let us label the proposed gauge-invariant Dirac fermion in the IR theory by $\Psi$. [The notation for the UV d.o.f. in the $S U(2)$ gauge theory is defined in Eq. (62).] The massless $\Psi$ theory describes a phase transition from the $n=0$ to the $n=-1$ state in the AIII class. Therefore, the $\Psi$ fermion should carry the following quantum number under the global symmetry $U(1) \times Z_{2}^{T}$ :

$$
\begin{gathered}
U(1): \Psi \rightarrow e^{i \theta} \Psi, \\
Z_{2}^{T}: \Psi \rightarrow-\gamma_{0} \gamma_{5} \Psi^{\dagger} .
\end{gathered}
$$

The "-" sign in the $Z_{2}^{T}$ transformation has physical consequences [83]. (Notice that no linear transformation of the fermion field can change this sign.) The convention is that a gapless Dirac fermion with the " + " $Z_{2}^{T}$ transformation describes a phase transition from the $n=0$ to the $n=1$ state in the AIII class. Correspondingly, a Dirac fermion with the " $-" Z_{2}^{T}$ transformation describes a transition from the $n=0$ to the $n=-1$ state.

By matching symmetry quantum numbers, the IR Dirac fermion operator $\Psi$ in terms of the UV d.o.f. is

$$
\Psi \sim \epsilon^{a b c}\left(i \bar{\psi}_{a} \psi_{b}\right)\left(\gamma_{5} \psi_{c}\right)-\epsilon^{a b c}\left(\bar{\psi}_{a} i \gamma_{5} \psi_{b}\right) \psi_{c} .
$$

The right-hand side is an $S U(2)$ gauge-singlet operator. The global $U(1)$ quantum number obviously matches. The $\bar{\psi}_{a} \psi_{b}$ is a Lorentz scalar, and $\bar{\psi}_{a} i \gamma_{5} \psi_{b}$ is a Lorentz pseudoscalar. The reason for choosing this specific combination of the scalar and pseudoscalar in the mapping is twofold. First, it is chosen to match the time-reversal transformation of the $\Psi$ fermion. Second, as we discuss later, with such a combination, the single Dirac fermion theory matches the 't Hooft anomalies of the emergent symmetries in the $S U(2)$ gauge theory. Let us look at the time-reversal symmetry first. We can check explicitly that the $\Psi$ in Eq. (89) satisfies the transformation in Eq. (88). First of all, let us write down $\Psi^{\dagger}$ :

$$
\Psi^{\dagger} \sim \epsilon^{a b c}\left(-i \bar{\psi}_{b} \psi_{a}\right)\left(\gamma_{5} \psi_{c}^{\dagger}\right)-\epsilon^{a b c}\left(i \bar{\psi}_{b} \gamma_{5} \psi_{a}\right) \psi_{c}^{\dagger} .
$$

Recall that the time-reversal action on the $\psi$ fermions is $\psi \rightarrow \gamma_{0} \gamma_{5} \psi^{\dagger}$. Also notice that the scalar $\bar{\psi} \psi$ is invariant under time reversal, while the pseudoscalar $\bar{\psi} i \gamma_{5} \psi$ is odd under time reversal. Therefore, the transformation of $\Psi$ is

$$
\begin{aligned}
Z_{2}^{T}: \Psi & \rightarrow \epsilon^{a b c}\left(-i \bar{\psi}_{b} \psi_{a}\right) \gamma_{5} \gamma_{0} \gamma_{5} \psi_{c}^{\dagger}-\epsilon^{a b c}\left(-\bar{\psi}_{b} i \gamma_{5} \psi_{a}\right) \gamma_{0} \gamma_{5} \psi_{c}^{\dagger} \\
& =-\gamma_{0} \gamma_{5}\left(\epsilon^{a b c}\left(-i \bar{\psi}_{b} \psi_{a}\right) \gamma_{5} \psi_{c}^{\dagger}-\epsilon^{a b c}\left(i \bar{\psi}_{b} \gamma_{5} \psi_{a}\right) \psi_{c}^{\dagger}\right) \\
& =-\gamma_{0} \gamma_{5} \Psi^{\dagger},
\end{aligned}
$$

which is indeed what we want. We partially list the gauge-invariant Lorentz scalar and spinor operators in Appendix III.

Since the operator $\bar{\Psi} \Psi$ and $\sum_{a=1}^{3} \bar{\psi}^{a} \psi^{a}$ share the same quantum numbers under all the global symmetries, they will have finite overlap in the IR. The conjecture is that $\Psi$ is free in the IR. Therefore, the anomalous dimension for the $\sum_{a=1}^{3} \bar{\psi}^{a} \psi^{a}$ operator should be zero. This result could be checked in future numerical calculations.

\section{B. Emergent symmetries and anomalies}

For both the $S U(2)^{*}+N_{f}^{A}=1$ theory and the Dirac theory, the global $\mathcal{G}=U(1) \times Z_{2}^{T}$ symmetry is a nonanomalous symmetry of the system for all values of the mass $m$. When the system is tuned to the critical point at $m=0$, it has enlarged global symmetries. These emergent symmetries usually have 't Hooft anomalies. Coupling these emergent symmetries to background gauge fields will lead to an inconsistency in the theory that can be cured [144] by regarding the theory as living at the boundary of a higher-dimensional SPT phase. In this section, we compare the emergent symmetries and their anomalies of the two theories at their critical points.

For the massless $S U(2)^{*}+N_{f}^{A}=1$ theory in the UV, the emergent symmetry is $\mathcal{G}=\left\{\left[S U(2)_{f} \times Z_{8}^{A}\right] / Z_{2}\right\}$. The $S U(2)_{f}$ is a flavor rotation symmetry, and $Z_{8}^{A}$ is a discrete axial rotation. The meaning of these symmetries will be clear in a moment. To understand these symmetries, let us look at the theory in Eq. (62) without the gauge field $a_{\mu}$. We can write down the Dirac fermions in the Weyl basis (we use a different set of $\gamma$ matrices than we used previously), in which a single Dirac fermion can be written as two Weyl fermions with different chiralities,

$$
\psi=\left(\begin{array}{c}
\xi_{1} \\
i \sigma^{y} \xi_{2}^{\dagger}
\end{array}\right)
$$

Here, $\xi_{1}$ and $\xi_{2}$ are both two-component left-handed Weyl fermions. The $i \sigma^{y} \xi_{2}^{\dagger}$ is the particle-hole transformation of $\xi_{2}$, and it has the opposite chirality. We can decompose our three Dirac fermions in Eq. (62) into six left-handed Weyl fermions (after a particle-hole transformation). The Lagrangian can be written as

$$
\mathcal{L}=i \sum_{a=1}^{3} \sum_{\alpha=1}^{2} \xi_{a, \alpha}^{\dagger} \bar{\sigma}_{\mu} \partial_{\mu} \xi_{a, \alpha}, \quad \bar{\sigma}_{\mu}=\{1,-\sigma\} .
$$

The largest unitary symmetry on the system is $U(6)$. Next, we want to gauge the diagonal $S U(2)$ subgroup of the $U(6)$ 
symmetry. Since the fermions are in the spin-1 representation, the gauge rotations on the Weyl fermions are $S O(3)$ rotations,

$$
S O(3)_{g}: \xi_{i, \alpha} \rightarrow O_{i j} \xi_{j, \alpha}, \quad \text { with } \quad O \in S O(3) .
$$

For convenience, we use $S O(3)_{g}$ to denote the gauge group in the following. [But keep in mind that eventually this is an $S U(2)$ gauge field because of the spin-1/2 spectator field.] The $U(6)$ symmetry can be decomposed as $U(6) \supset$ $\left\{[S U(3) \times S U(2) \times U(1)] / Z_{3} \times Z_{2}\right\} \supset\left\{\left[S O(3)_{g} \times S U(2) \times\right.\right.$ $\left.U(1)] / Z_{2}\right\}$. Therefore, the global symmetry left after gauging is naively $\left\{[S U(2) \times U(1)] / Z_{2}\right\}$. The $S U(2)$ is a flavor rotation; therefore, we denote it as $S U(2)_{f}$. Its action on the Weyl fermions is

$$
S U(2)_{f}: \xi_{i, \alpha} \rightarrow U_{\alpha \beta} \xi_{i, \beta}, \quad \text { with } \quad U \in S U(2) .
$$

The six Weyl fermions form three fundamental representations of the $S U(2)_{f}$. The action of the $U(1)$ symmetry is

$$
U(1)_{A}: \xi_{i, \alpha} \rightarrow e^{i \phi} \xi_{i, \alpha} .
$$

Because of the particle-hole transformation on the $\xi_{i, 2}$ fields, this $U(1)$ rotation is the $\gamma_{5}$ rotation of the original Dirac fermion, which is usually called the axial rotation. We label it as $U(1)_{A}$. The familiar charge $U(1)$ rotation of the Dirac fermion is now the $S_{z}$ rotation of $S U(2)_{f}$.

The $U(1)_{A}$ suffers from chiral anomalies. It is explicitly broken down to $Z_{8}$ after considering the mixed anomalies with $S O(3)_{g}$. This result is seen in the following anomaly equations:

$$
\begin{gathered}
\partial_{\mu} j^{\mu 5}=\operatorname{Tr}_{S O(3)}\left(\frac{F^{S O(3)}}{2 \pi} \wedge \frac{F^{S O(3)}}{2 \pi}\right) \\
=4 \operatorname{Tr}_{S U(2)}\left(\frac{F^{S U(2)}}{2 \pi} \wedge \frac{F^{S U(2)}}{2 \pi}\right), \\
\int_{Y^{4}} \partial_{\mu} j^{\mu 5}=2 p_{1}(S O(3))=8 p_{1}(S U(2)) \in 8 \mathbb{Z} .
\end{gathered}
$$

The first part of the equation is the standard Fujikawa calculation for Abelian anomalies [81]. In the second part, we use the relation between the Pontryagin classes in the $S O(3)$ and $S U(2)$ groups. The Pontryagin class of $S U(2)$ counts the instanton number of the $S U(2)$ gauge field and takes its value in integers. The equation means the axial charge will change by 8 if we insert an $S U(2)$ instanton configuration with winding number 1 . Therefore, the axial charge is only well defined up to 8 . The $U(1)_{A}$ is broken down to $Z_{8}^{A}$.

Note that there is no mixed anomaly between $S U(2)_{f}$ and $S O(3)_{g}$. The divergence of the $S U(2)_{f}$ current is $\partial_{\mu} j_{\alpha}^{\mu}=\frac{1}{24 \pi^{2}} \operatorname{Tr}\left[\sigma_{\alpha} \partial_{\kappa} \epsilon^{\kappa \lambda \mu \nu}\left(A_{\lambda} \partial_{\mu} A_{\nu}+\frac{1}{2} A_{\lambda} A_{\mu} A_{\nu}\right)\right]=0$,

where $A_{\mu}=\sum_{a=1}^{3} A_{\mu}^{a} T_{a}, T_{a}$ 's are $S O(3)$ generators, and $\sigma_{\alpha}$ 's are Pauli matrices. The anomaly equations are determined by calculating certain triangle loop diagrams $[81,145]$. The essential part of the right-hand side of the equation involves the trace of three symmetry generators. In this case, it is clearly zero because the $S O(3)$ generator and the $S U(2)$ generator act on different spaces. In the flavor space, the trace of an $S U(2)$ generator is zero. This result tells us that the $S U(2)_{f}$ is still a symmetry after gauging the $S O(3)_{g}$. Thus, we see that the global symmetry for the critical $S U(2)^{*}+N_{f}^{A}=1$ theory is $\mathcal{G}=\left\{\left[S U(2)_{f} \times Z_{8}^{A}\right] / Z_{2}\right\}$.

In the infrared limit, it is possible that the $Z_{8}$ symmetry is dynamically enhanced to $U(1)$. There are many examples of such a phenomenon in $2+1-\mathrm{D}$ deconfined quantum critical points $[4,19,146]$. Though we cannot be sure that this enlargement actually happens in our case, we are encouraged by the matching of anomalies with the free Dirac theory at its massless point [which has emergent $U(2)=\left\{[S U(2) \times U(1)] / Z_{2}\right\}$ symmetry $]$ discussed below [147]. Henceforth, in talking about the free Dirac theory, we simply treat the $Z_{8}$ axial symmetry of the gauge theory as though it is a $U(1)$ symmetry. A proper discussion of the anomalies involving the $Z_{8}$ without this simplification is given in Ref. [59].

Now, let us consider the anomaly structure for $\mathcal{G}$. First, we discuss the t' Hooft anomaly of $S U(2)_{f}$. The $S U(2)_{f}$ itself has no perturbative anomaly, but it has the global Witten anomaly. The Witten anomaly is a $Z_{2}$ anomaly [148] which depends only on the parity of the number of $S U(2)_{f}$ fundamental Weyl fermions. Here, we have three $S U(2)_{f}$ fundamental Weyl fermions. Therefore, they carry the $S U(2)$ Witten anomaly. Dynamically gauging the $S U(2)_{f}$ symmetry will lead to a vanishing partition function.

The $Z_{8}$ symmetry has a self-'t Hooft anomaly and mixed anomalies with $S U(2)_{f}$ and gravity. The anomaly is summarized in the following equation:

$\partial_{\mu} j_{A}^{\mu}=3\left(\frac{f_{A}}{2 \pi} \wedge \frac{f_{A}}{2 \pi}\right)+\frac{3}{2} \operatorname{Tr}_{S U(2)}\left(\frac{F_{S U(2)_{f}}}{2 \pi} \wedge \frac{F_{S U(2)_{f}}}{2 \pi}\right)-\frac{6}{8} \sigma$.

Next, we look at the IR Dirac fermion $\Psi$ at its massless point. In the Weyl basis, the Dirac theory reads

$$
\mathcal{L}=i \sum_{\alpha=1}^{2} \eta_{\alpha}^{\dagger} \bar{\sigma}_{\mu} \partial_{\mu} \eta_{\alpha}, \quad \bar{\sigma}_{\mu}=\{1,-\sigma\}
$$

where $\eta_{1}$ and $\eta_{2}$ are both left-handed Weyl fermions. According to our dictionary in Eq. (90), the $\eta$ fermions 
can be written as composite operators from $\xi$ fermions in the $S U(2)$ gauge theory.

$$
\begin{aligned}
\eta_{1} & \sim \epsilon^{a b c} i\left(\xi_{a, 2} i \sigma^{y} \xi_{b, 1}\right) \xi_{c, 1}, \\
\eta_{2} & \sim \epsilon^{a b c} i\left(\xi_{a, 1} i \sigma^{y} \xi_{b, 2}\right) \xi_{c, 2} .
\end{aligned}
$$

The theory manifestly has $\mathcal{G}^{A}=\left\{\left[S U(2)_{f} \times U(1)_{A}\right] / Z_{2}\right\}$ symmetry. These symmetries are in one-to-one correspondence with the emergent symmetries in the $S U(2)^{*}+N_{f}^{A}=1$ theory if, as we assumed, the $Z_{8}$ symmetry of the latter theory is enhanced to $U(1)$ in the gapless sector of the proposed IR theory. The $S U(2)_{f}$ transformation is

$$
S U(2)_{f}: \eta_{\alpha} \rightarrow U_{\alpha \beta} \eta_{\beta}, \quad \text { with } \quad U \in S U(2) .
$$

This transformation is consistent with the definition in Eq. (102). From the definition, the $\eta$ fermions carry charge 3 under the axial $U(1)_{A}$ symmetry in the $S U(2)$ gauge theory.

$$
U(1)_{A}: \eta_{\alpha} \rightarrow e^{i 3 \phi} \eta_{\alpha}
$$

This property is crucial for matching the anomalies with the UV theory.

Now, we study the 't Hooft anomalies of the emergent symmetry. First, the $S U(2)_{f}$ symmetry has the same global Witten anomaly [148] because we have a single $S U(2)_{f}$ fundamental Weyl fermion. The anomalies associated with $U(1)_{A}$ are summarized in the following anomaly equation:

$\partial_{\mu} j_{A}^{\mu}=27\left(\frac{f_{A}}{2 \pi} \wedge \frac{f_{A}}{2 \pi}\right)+\frac{3}{2} \operatorname{Tr}_{f}\left(\frac{F_{S U(2)_{f}}}{2 \pi} \wedge \frac{F_{S U(2)_{f}}}{2 \pi}\right)-\frac{6}{8} \sigma$,

where the coefficients 27 and 3 precisely come from the fact that the $\Psi$ fermions carry charge 3 under the axial $U(1)_{A}$ symmetry. This fact will match the anomalies in Eq. (100) if we consider the discrete $Z_{8}$ axial symmetry instead of the $U(1)_{A}$. This result indicates that the lowenergy theory cannot be a simple Dirac fermion but needs some additional sector that remembers that the $U(1)_{A}$ is broken down to $Z_{8}$.

\section{The 1-form symmetry anomalies and the additional $Z_{2}$ topological order}

Thus far, we argued that the IR Dirac fermion $\Psi$ matches almost all the 0 -form symmetry and anomalies in the UV theory. Now, we focus on the 1 -form $\left(Z_{2}\right)_{1}$ symmetry of the system in the infinitely massive spectator limit. The IR Dirac fermion does not have the 1-form symmetry. As shown in Ref. [59], this $\left(Z_{2}\right)_{1}$ symmetry has mixed anomalies with the $Z_{8}$ and with gravity. Therefore, there must be other d.o.f. in the IR that carry the 1-form symmetry and its anomalies.

The anomalies involving the $Z_{2} 1$-form symmetry have two pieces according to Ref. [59]. The first part is a mixed anomaly between the $Z_{2}$ 1-form symmetry and the $Z_{8}^{A}$ discrete axial symmetry. Let us call this the type I anomaly. The mixed anomaly means that dynamically gauging the $Z_{2} 1$-form symmetry will break the $Z_{8}$ down to $Z_{4}$ on a spin manifold and $Z_{2}$ on a nonspin manifold. Formally, we can couple the system to a background 2-form $Z_{2}$ gauge field $B$. By definition, a symmetry operation on a quantum system should preserve the partition function. However, in this system, in the presence of the 2-form background gauge field $B$, the partition function is no longer invariant under $Z_{8}$ axial rotation. The $k$ th element of $Z_{8}$ axial rotation will shift the partition function by a phase $\exp \left\{i[(\pi k) / 2] \int_{Y^{4}} \mathcal{P}(B)\right\}$, where $\mathcal{P}(B)$ is the Pontryagin square of $B$. On a spin manifold, $\int_{Y^{4}} \mathcal{P}(B)$ is quantized as an even number [112]. Therefore, the $Z_{8}$ is broken down to $Z_{4}$ by the mixed anomaly. On a nonspin manifold, $\int_{Y^{4}} \mathcal{P}(B)$ is an arbitrary integer [112], and the axial symmetry is then broken down to $Z_{2}$. The second anomaly is more abstract. It is a mixed anomaly between the $Z_{2}$ 1-form symmetry and geometry. We call this the type II anomaly. This anomaly has the following formal interpretation. We again couple the system to a 2 -form $Z_{2}$ gauge field $B$. The 2-form gauge field is a $Z_{2}$ gauge field, which means that a redefinition of the 2-form gauge field, $B \rightarrow B+2 x$, with $x$ another 2-form $Z_{2}$ gauge field, should not change the partition function of the system. However, in this theory, such a redefinition changes the partition function by a factor $\exp \left[i \pi x \cup w_{2}^{T Y}\right]$, which can be -1 on a nonspin manifold.

It is useful for us to have a more concrete physical picture for both types of anomalies. The type I anomaly in the UV has the following physical interpretation. Let us remind ourselves from Eq. (98) that the change of axial charge is equal to 8 times the instanton number of the $S U(2)$ gauge field. Coupling the $S U(2)$ gauge theory to the $Z_{2}$ 2-form gauge field $B$ is effectively turning the $S U(2)$ bundle into an $S O(3)$ bundle, which has magnetic monopole excitations. The instanton number for the $S U(2)$ bundle is quantized to be an integer. However, when we extend the $S U(2)$ bundle to the $S O(3)$ bundle, we have new field configurations involving the $S O(3)$ monopoles, and the quantization of the instanton number is changed. On spin manifolds, the $S O(3)$ instanton number is quantized as half-integer. On nonspin manifolds, the smallest $S O(3)$ instanton number can be one quarter.

The $1 / 2$ instanton event for the $S O(3)$ bundle has the following physical picture. We take two $2 \pi$ magnetic flux loops [149] initially separated in space and then move them across each other to form a link $[150,151]$. This space-time process produces the $1 / 2$ instanton [152].

We can now give a physical description of the mixed anomaly between $Z_{8}$ and $\left(Z_{2}\right)_{1}$. We assign an axial charge 
of 4 to two $2 \pi S O(3)$ flux loops that have linking number 1. The instanton event changes this linking number and hence breaks the $Z_{8}$ to $Z_{4}$. On a nonspin manifold, for example $C P^{2}$, there is an even smaller instanton event. It can be roughly thought of as creating a self-linking of the $2 \pi S O(3)$ magnetic flux.

The type II anomaly involves the second Stiefel-Whitney class of the tangent bundle, which detects the spin structure of the base manifold. This anomaly tells us that there is an ambiguity on the quantum statistics of the $2 \pi S O(3)$ monopole. Below, we build on these physical characterizations to augment the free Dirac theory with a gapped sector that enables the matching of the 1-form anomalies.

Note that the extra anomalies discussed in this section are of the discrete unitary symmetry $Z_{8} \times\left(Z_{2}\right)_{1}$. For ordinary 0 -form discrete unitary symmetries (at or above $2+1-\mathrm{D})$, it is known that their anomalies can always be satisfied by a symmetry-preserving gapped topological order [153]. Inspired by this idea, we ask if there can be some symmetry-preserving [154] $3+1$-D topological order that captures the anomalies of $Z_{8} \times\left(Z_{2}\right)_{1}$. Furthermore, note that with anomalous 0 -form symmetry, the charge particles will be fractionalized into partons that carry projective representations of the symmetry. Here, the anomalous $Z_{2}$ 1 -form symmetry acts on loops. Thus, we are led to search for a topological ordered state of matter that has "fractionalized" loop excitations. A short introduction and an example of such a fractionalized loop phase are given in Appendix F. Now, we describe a postulated topological order that can match the anomalies associated with the 1 -form symmetry. It has the following properties.

(1) It is a "loop-fractionalized" topological phase that preserves the $\left(Z_{2}\right)_{1} \times Z_{8}$ symmetry.

(2) The specific theory is a $Z_{2}$ gauge theory, where the "microscopic" loops (we call them $2 \pi$-flux loops) have fractionalized into two $\pi$-flux loops. The physical manifestation of the $\left(Z_{2}\right)_{1}$ is that the $2 \pi$ flux loops are unbreakable.

(3) The $Z_{2}$ gauge charge carries fermionic statistics.

(4) Two linked electric loops of the $Z_{2}$ gauge theory carry axial charge 16 . These loops can unlink dynamically as there are sources for the electric loops. The linked loops are therefore mixed in with the unlinked loops by the Hamiltonian. The groundstate wave function contains all electric loop configurations (linked or unlinked); hence, the state has global $Z_{16}$ symmetry.

(5) Each electric loop should be thought of as a ribbon. A self-linked loop is assigned axial charge of 8 . Events in the theory that create a single such selflinked loop will break the axial symmetry to $Z_{8}$.

Now, let us explain why this topological order can match the $Z_{8} \times\left(Z_{2}\right)_{1}$ anomalies. The Fermi statistics of electric charge- 1 objects ensures that the $\left(Z_{2}\right)_{1}$ symmetry has the right mixed anomaly with gravity. Gauging the $\left(Z_{2}\right)_{1}$ symmetry introduces electric charge- $1 / 2$ particles. Since the fusion result of two charge- $1 / 2$ particles must be the charge- 1 particle which is a fermion, these charge- $1 / 2$ particles have indefinite statistics. In contrast, in a strictly $3+1-\mathrm{D}$ system, it should be possible to assign definite statistics to these particles. This case is the manifestation of the mixed anomaly between $\left(Z_{2}\right)_{1}$ and geometry.

Introducing electric charge- $1 / 2$ particles into the theory implies that the system must also allow strength- $1 / 2$ electric loops. These 1/2-strength electric loops can form links. A link of two $1 / 2$ electric loops will carry axial charge 4. However, as there are sources for these loops, the linking number can change dynamically. An event in which two linked strength- $1 / 2$ electric loops is created changes the axial charge by 4 . This result breaks the axial symmetry down to $Z_{4}$. We also need to consider a single strength- $1 / 2$ loop that is self-linked. As a self-linked strength-1 loop is assigned axial charge 8 , a self-linked charge-1/2 loop should be assigned axial charge 2 . Dynamically, again, the self-linking number can change as there are sources for the loops. It follows that an event where a self-linked strength$1 / 2$ electric loop is created changes the axial charge by 2 . Therefore, the axial symmetry is broken down to $Z_{2}$. These results precisely match the mixed anomaly between $\left(Z_{2}\right)_{1}$ and $Z_{8}$ axial symmetry.

To recap, the proposed low-energy theory is a free massless Dirac fermion augmented with the topologically ordered state just described. What we have argued is that this theory has the same global symmetries, the same local operators, and the same anomalies as the $S U(2)$ gauge theory with an $N_{f}^{A}=1$ adjoint Dirac fermion (and no spectator fundamental scalar). Of course, we do not know if the gauge theory really flows to the free Dirac + topological theory, but we are encouraged by these checks. Alternate possibilities have been discussed in Ref. [59].

Let us now introduce a finite mass for the spin- $1 / 2$ spectator fields in our UV theory. With a finite-mass spectator, the $Z_{2} 1$-form symmetry is explicitly broken. Physically, this means that the $2 \pi$-flux loops can be broken dynamically. The question is whether the $Z_{2}$ topological order we described is immediately destroyed dynamically by a finite but large spectator mass. In our case, since the topological order is in a fractionalized-loop phase, the $\pi$-flux loops still cannot break, and they remain nontrivial excitations in our system. Therefore, with a large but finite spectator mass, the $Z_{2}$ topological order is still stable.

If the low-energy theory of massless $S U(2)+N_{f}^{A}=1$ theory with finite spectator mass is indeed a free Dirac fermion plus a decoupled $Z_{2}$ topological order, then the phase diagram of the theory will be as shown in Fig. 10. Since the $Z_{2}$ topological order is stable against small perturbations, it will survive until a critical fermion mass $m_{c}$. The phase transition at $m=0$ occurs entirely in the gapless free Dirac sector, and it describes the topological phase transition between the $n=0$ and either $n=-1$ or 


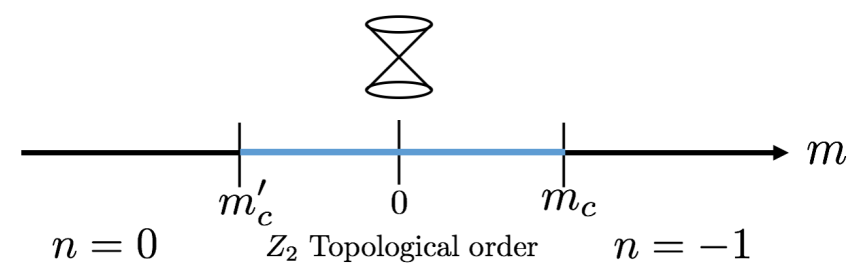

FIG. 10. If the $S U(2)+N_{f}^{A}=1$ theory is dual to a single Dirac fermion supplemented by a $Z_{2}$ topological order, the adjoint fermion mass will not drive the system to the large-mass confined phase immediately as the $Z_{2}$ topological order is stable against small perturbations. Increasing the fermion mass, the $Z_{2}$ topological order should go through another phase transition to the confined phases.

$n=3$ class AIII topological superconductors (depending on the time-reversal properties of the spectators).

\section{DISCUSSION}

From a condensed matter perspective, the main results in this paper are the numerous examples of unusual quantum critical phenomena. Here, we briefly describe some general lessons we can learn.

(1) The possibility of multiple universality classes for the same phase transition (of which we found many examples) arises in many different contexts. As far as we are aware, previous examples of this phenomenon are known only in systems with quenched randomness (for instance, the $\pm J$ spin glass). An important context is at heavy-electron quantum critical points between a Fermi liquid and an antiferromagnetic metal. The standard Moriya-HertzMillis "spin-density-wave" theory for the onset of antiferromagnetism in a metal has difficulties with the phenomenology observed in some systems. Alternate theories invoke the idea of Kondo breakdown and posit a distinct universality class. However, it has never been very clear whether the resulting antiferromagnetic phase is necessarily sharply distinct from the one obtained through the spin-density-wave route. It is interesting, therefore, to contemplate that the heavy-fermi-liquid-to-antiferromagnetic-metal transition may admit (at least) two distinct universality classes between the same two phases.

(2) The existence of "unnecessary quantum critical points" should be kept in mind when the question of which two phases are separated by a quantum critical point is posed in some condensed matter system. The two phases may not actually be sharply distinct.

(3) We found a number of examples of band-theoryforbidden quantum critical points between band insulators. These examples raise the general question of what the criteria are-beyond free fermion theory-for which transitions between free fermion topological phases are allowed to be continuous. Consider, for instance, integer quantum Hall transitions of electrons. At the free fermion level, it is well known that the quantized values of both electrical and thermal Hall conductivities will generically jump by 1 at a continuous transition. Is this still true in the presence of interactions?

(4) Previous examples of deconfined quantum critical points have been shown in $2+1$-D systems (as far as we are aware). It is encouraging that we have been able to find a number of examples of this phenomenon in $3+1-D$, which furthermore have emergent non-Abelian gauge fields at the critical point. All of our examples describe transitions between gapped short-range entangled phases (possibly distinguished as SPT phases). It will be interesting to search for other 3+1-D examples-like in the Neel-valence bond solid transition of 2+1-D magnets-where a Landau forbidden transition occurs between two symmetry-broken Landau-allowed phases.

(5) Continuous phase transitions between SPT phases in $3+1-D$ have not been explored much (beyond free fermion theory). The examples we have found and the resulting novel phenomena should give impetus to a systematic study of such transitions.

From a high-energy perspective, one of our results is to provide an interpretation of some massless gauge theories as quantum critical points. We saw that, even when the gauge theory is IR-free, it has an interesting place in the phase diagram as a deconfined quantum critical point. Perhaps the most interesting aspect (for quantum field theorists) is our discussion of the possible duality of the $S U(2)$ gauge theory with a massless $N_{f}=1$ adjoint (Dirac) fermion (and a massive fundamental boson) to a free massless Dirac fermion with an additional decoupled topological field theory. It will be interesting to scrutinize this possibility through numerical studies of the gauge theory.

\section{ACKNOWLEDGMENTS}

We thank Max Metlitski for innumerable discussions and constant encouragement. We also thank Nati Seiberg for a crucial conversation that pointed us toward the examples of multiple universality classes. Z. B. thanks Liujun Zou, Yuji Tachikawa, Juven Wang, Clay Cordova, and Chao-ming Jian for numerous stimulating discussions. We thank the participants of the Aspen Winter Conference on "Field Theory Dualities and Strongly Correlated Matter" for many useful discussions and feedback. This work was supported by National Science Foundation (NSF) Grant No. DMR1608505, and partially by the Simons Foundation (T. S.). Part of this work was performed at the Aspen Center for Physics, which is supported by NSF Grant No. PHY1607611. Z. B. acknowledges support from MIT. We also 
acknowledge the MIT open access article publication subvention fund.

\section{APPENDIX A: INSTANTON NUMBER}

By definition, a Yang-Mills instanton is a solution of the classical Euclidean equations of motion with finite action. To find solutions with finite action, we require that the field strength tends to zero at infinity sufficiently fast. Hence, the gauge field asymptotically approaches a pure gauge. All pure-gauge configurations, namely, $A=U^{-1} d U$, at infinity are classified by

$$
\pi_{3}(\mathcal{G})=\mathbb{Z}
$$

which is characterized by an integer number, the instanton number. First, consider gauge configurations on $\mathbb{R}^{4}$, which become pure gauge at asymptotic infinity. Given a group $\mathcal{G}$, the instanton number of any such gauge configuration on $\mathbb{R}^{4}$ is an integer multiple of a minimal positive number. This minimal instanton corresponds to the generator of $\pi_{3}(\mathcal{G})=\mathbb{Z}$. It is customary in the literature to normalize this minimal instanton so that it has instanton number 1 . If $\mathcal{G}$ has a discrete $\mathbb{Z}_{2}$ subgroup, since $\pi_{2}\left(\mathbb{Z}_{2}\right)=\pi_{3}\left(\mathbb{Z}_{2}\right)=$ $\mathbb{Z}_{1}$, we have

$$
\pi_{3}\left(\mathcal{G} / \mathbb{Z}_{2}\right)=\pi_{3}(\mathcal{G})
$$

which indicates that $\mathcal{G} / \mathbb{Z}_{2}$ and $\mathcal{G}$ share the same generator for instantons. For any non-Abelian group $\mathcal{G}$, an instanton of minimal charge can be obtained by embedding a minimal instanton of $S U(2)$ through an appropriate isomorphism $S U(2) \rightarrow \mathcal{G}$, which is obtained by picking a sub$S U(2)$ algebra generated by a long root in the Lie algebra of $\mathcal{G}$. For continuous group $\mathcal{G}$, the instanton number can be calculated from an integral of a local density,

$$
l_{\mathcal{G}}=c_{R} \int_{Y_{4}} \operatorname{Tr}_{R}\left(\frac{F}{2 \pi} \wedge \frac{F}{2 \pi}\right)
$$

where $R$ denotes a specific representation we can freely choose, and the coefficient $c_{R}$ is chosen to make sure that $l_{\mathcal{G}}=1$ for the minimal instanton configuration. Particularly, $c_{R}$ can be determined by embedding the minimal $S U(2)$ instanton into $\mathcal{G}$ and evaluating the expression above. If we use adjoint representation in Eq. (A3), the normalization coefficient $c_{R}$ will only depend on the Lie algebra of $\mathcal{G}$ but not the global structure of the group [155]. Therefore, the formula gives the same result for $\mathcal{G}$ and $\mathcal{G} / \mathbb{Z}_{2}$, namely, $l_{\mathcal{G}}=l_{\mathcal{G} / \mathbb{Z}_{2}}$. All the instanton numbers we used in the main text are normalized in this way.

Now, let us discuss the relation between the Pontryagin classes and the instanton numbers of $S U(N), S O(N)$, and $\operatorname{Sp}(N)$ groups. The first Pontryagin class of a group $\mathcal{G}$ is defined with its fundamental representation as follows:

$$
p_{1}(\mathcal{G})=\frac{1}{2} \int_{Y_{4}} \operatorname{tr}_{f}\left(\frac{F}{2 \pi} \wedge \frac{F}{2 \pi}\right) .
$$

For the $S U(2)$ group, we get exactly 1 from Eq. (A4) if we plug in the minimal instanton configuration. This result indicates that the first Pontryagin class is equal to the instanton number for the $S U(2)$ group, namely, $p_{1}(S U(2))=l_{S U(2)}$. This is a starting point. Now, consider the $S U(N)$ and $S p(N)$ groups. The minimal instanton number is achieved by embedding the minimal $S U(2)$ instanton configuration in the upper-left corner in the gauge configuration as follows:

$$
A_{\mu}=\left[\begin{array}{cc}
A_{\mu}^{S U(2)} & 0 \\
0 & 0
\end{array}\right]
$$

It is obvious that we will get 1 if plug this into Eq. (A4). Therefore, for $S U(N)$ and $S p(N)$, the first Pontryagin class is equal to their instanton number.

$$
\begin{aligned}
& p_{1}(S U(N))=l_{S U(N)}, \\
& p_{1}(S p(N))=l_{S p(N)} .
\end{aligned}
$$

The case for the Pontryagin class of the $S O(N)$ group is a little complicated. It is defined as having the same form in Eq. (A4) normalized with the vector representation of $S O(N)$. For $S O(3)$, we can only embed the $S U(2)$ instanton configuration into the $S O(3)$ gauge configurations using the $S U(2)$ adjoint representation. Because of this embedding, for a minimal $S U(2)$ instanton configuration, $p_{1}(S O(3))$ is actually equal to 4 . Hence, $p_{1}(S O(3))$ is equal to 4 times the instanton number,

$$
\begin{aligned}
p_{1}(S O(3)) & =\frac{1}{2} \int_{Y_{4}} \operatorname{tr}_{S O(3)}\left(\frac{F}{2 \pi} \wedge \frac{F}{2 \pi}\right) \\
& =4 \frac{1}{2} \int_{Y_{4}} \operatorname{tr}_{S U(2)}\left(\frac{F}{2 \pi} \wedge \frac{F}{2 \pi}\right) \\
& =4 l_{S U(2)}=4 l_{S O(3)} .
\end{aligned}
$$

The embedding for $S O(N)$ with $N>3$ is different. We make use of the fact that $S O(N) \supset S O(4)=$ $S U(2) \times S U(2) / \mathbb{Z}_{2}$, and we embed the $S U(2)$ instanton configuration into one of the $S U(2)$ subgroups of $S O(4)$. With this embedding, it is easy to verify that $p_{1}(S O(N))$ is equal to 2 if we put in a minimal instanton configuration. Therefore,

$$
p_{1}(S O(N>3))=2 l_{S O(N)} .
$$

The $\theta$ angle of $3+1-\mathrm{D}$ gauge theories is usually defined so that a configuration of instanton number 1 contributes to the Euclidean action by the phase $\exp (i \theta)$. 


\section{APPENDIX B: A 2 + 1D EXAMPLE OF UNNECESSARY CONTINUOUS PHASE TRANSITION}

In the same spirit as the $3 d$ examples, let us give an example in $2 d$. We consider the trivial-to-topological phase transition of the $p \pm i p$ superconductor system with $Z_{2} \times$ $Z_{2}^{T}$ symmetry. The low-energy Hamiltonian near the phase transition is as follows:

$$
H_{\times 1}=\int d^{2} x \chi^{T}\left(i \partial_{x} \sigma^{10}+i \partial_{y} \sigma^{30}+m \sigma^{23}\right) \chi,
$$

where the $Z_{2}$ symmetry, $Z_{2}: \chi \rightarrow \sigma^{03} \chi$, is the relative fermion parity symmetry of the two layers. Time-reversal symmetry, $\mathcal{T}: \chi \rightarrow i \sigma^{21} \chi$, exchanges the \pm layers. The two symmetries together only admit the mass term in Eq. (B4), which guarantees that there is a generic phase transition described by free Majorana fermions in the bulk. The edge of the system consists of helical Majorana modes described by the following equation:

$$
H_{\text {edge }}=\int d x \chi^{T}\left(i \partial_{x} \sigma^{3}\right) \chi
$$

The $Z_{2}$ and time-reversal transformations are

$$
Z_{2}: \chi \rightarrow \sigma^{3} \chi, \quad Z_{2}^{T}: \chi \rightarrow i \sigma^{2} \chi .
$$

We can introduce a mass term on the boundary $m_{b} \chi^{T} \sigma^{2} \chi$, which breaks both $Z_{2}$ and $Z_{2}^{T}$ symmetries but preserves a different time-reversal symmetry $\tilde{Z}_{2}^{T}: \chi \rightarrow-\sigma^{1} \chi$ (which is the original $Z_{2}^{T}$ transformation followed by the $Z_{2}$ transformation). The domain wall of the $Z_{2}$-breaking mass term traps a Majorana zero mode, labeled by $\gamma$. The $\tilde{Z}_{2}^{T}$ symmetry will not change the domain-wall background, and it just acts trivially on the zero modes, namely, $\tilde{Z_{2}^{T}}: \gamma \rightarrow \gamma$.

Now let us consider eight copies of the same system and impose an $S O(7)$ symmetry that rotates these eight copies in the spinor representation. This symmetry only allows a uniform mass term. The low-energy theory near the phase transition is as follows:

$H_{\times 8}=\int d^{2} x \sum_{i=1}^{8} \chi_{i}^{T}\left(i \partial_{x} \sigma^{10}+i \partial_{y} \sigma^{30}+m \sigma^{23}\right) \chi_{i}$.

When $m$ is tuned from negative to positive, the system goes through a continuous phase transition described by bulkfree Majorana fermions. This transition is stable against small interactions. Our goal now is to show that the $m<0$ and $m>0$ phases are in fact the same phase. We can always regularize the system such that the $m<0$ phase is trivial. In the $m>0$ phase, the natural edge state has eight copies of helical Majorana modes with $Z_{2} \times Z_{2}^{T} \times S O(7)$ symmetry. We argue that the boundary modes can be gapped out while preserving all the symmetries, which indicates that the $m>0$ phase is actually topologically trivial.

To this end, we first break the $Z_{2}$ and $Z_{2}^{T}$ symmetry on the edge by adding $m_{b} \sum_{i=1}^{8} \chi_{i}^{T} \sigma^{2} \chi_{i}$. Then, we proliferate the topological defects of this order parameter, namely, the domain walls, to restore a symmetric gapped edge. Since there are zero modes residing at the domain wall of the order parameter, we have to be careful about their condensation. The domain wall must have a single gapped ground state, and it has to be symmetric under the combined $\tilde{Z}_{2}^{T}$ symmetry. This result can be precisely achieved by the $S O(7)$-invariant Fidkowski-Kitaev interaction. Therefore, with this interaction, we can safely condense the domain wall to get a symmetric gapped edge state. Thus, the $m>0$ phase is topologically trivial. The phase diagram of the system is similar to the previous cases as shown in Fig. 6.

\section{APPENDIX C: FERMION ZERO MODES AND TIME-REVERSAL TRANSFORMATIONS}

In this Appendix, we consider a $2+1$-D Dirac fermion in a $2 \pi$-flux background and solve the zero-mode wave function. Then, we consider the time-reversal transformation on the zero mode.

Let us first write down the Hamiltonian for the 2+1-D Dirac fermion on a flat two-dimensional plane with a background gauge field,

$$
H=\psi^{\dagger}\left(\left(i \partial_{x}-A_{x}\right) \sigma^{x}+\left(i \partial_{y}-A_{y}\right) \sigma^{z}\right) \psi=\psi^{\dagger} \mathcal{D} \psi,
$$

where we take the Landau gauge $A_{x}=0, A_{y}=B x$. Notice that this is equivalent to the spherical geometry since the flat plane can be viewed as the infinite radius limit of the sphere. The time-reversal transformation for the fermion fields is

$$
Z_{2}^{T}: \psi \rightarrow i \sigma^{y} \psi^{\dagger}
$$

In component form, the time-reversal action is

$$
Z_{2}^{T}: \psi_{1} \rightarrow \psi_{2}^{\dagger}, \quad \psi_{2} \rightarrow-\psi_{1}^{\dagger} .
$$

This time-reversal transformation will flip the electric charge of the Dirac fermions but keep the magnetic flux background invariant. Therefore, it is meaningful to discuss the time-reversal transformation of the zero modes trapped in the flux background.

Consider the Dirac equation

$$
\mathcal{D} \psi=\left(i \partial_{x} \sigma^{x}+\left(i \partial_{y}-B x\right) \sigma^{z}\right) \psi=\varepsilon \psi .
$$

The usual trick to solve the Dirac equation is to square the Dirac operator to get 


$$
\left(p_{x}^{2}+\left(p_{y}-B x\right)^{2}+B \sigma^{y}\right) \psi=\varepsilon^{2} \psi .
$$

The spectrum for $\varepsilon^{2}$ is (in the unit with $\hbar=c=1$ )

$$
\varepsilon^{2}=|B|(2 n+1)-|B|, \quad n \in \mathbb{Z} .
$$

Notice that the zero-mode wave function depends on the sign of magnetic field $B$. Consequentially, the time-reversal transformations on the zero modes are different for $\pm B$. For $B>0$, the zero-mode operator is

$$
f_{+}=\left(\psi_{1}-i \psi_{2}\right) \phi_{0}\left(p_{y}, x\right),
$$

while for $B<0$, the zero-mode operator is

$$
f_{-}=\left(\psi_{1}+i \psi_{2}\right) \phi_{0}\left(p_{y}, x\right),
$$

where $\phi_{0}\left(p_{y}, x\right)$ is the wave function for the ground state of a harmonic oscillator.

Thus, we find that the time-reversal transformations for the zero modes are

$$
\begin{gathered}
Z_{2}^{T}: f_{+} \rightarrow\left(\psi_{2}^{\dagger}-i \psi_{1}^{\dagger}\right) \phi_{0}^{*}\left(p_{y}, x\right)=-i f_{+}^{\dagger} \\
f_{-} \rightarrow\left(\psi_{2}^{\dagger}+i \psi_{1}^{\dagger}\right) \phi_{0}^{*}\left(p_{y}, x\right)=i f_{-}^{\dagger}
\end{gathered}
$$

\section{APPENDIX D: BAND-THEORY-FORBIDDEN QUANTUM CRITICALITY BETWEEN TWO BAND INSULATORS: A SIMPLE EXAMPLE}

A system of free fermions can be in a gapped ground state. Such distinct states in any given system are labeled by topological invariants. Within free fermion theory, there are rules on what kinds of continuous phase transitions can occur between these distinct phases. Roughly speaking, the topological invariant jumps by the smallest possible amount in order for a direct second-order transition to be possible. The best-known example is in a system of fermions with charge- 1 under a global $U(1)$ symmetry in two space dimensions. Gapped free fermion ground states show an integer quantum Hall effect characterized by a quantized electrical Hall conductivity $\sigma_{x y}=n \in \mathbb{Z}$ and a quantized thermal Hall conductivity $\kappa_{x y}=\bar{c}\left[\left(\pi^{2} k_{B}^{2} T\right) / 3\right]$ with $\bar{c}=n$. These distinct phases can be realized within band theory by fully filling a band with Chern number $n$. In the absence of any other symmetries [156], within free fermion theory, continuous phase transitions between these distinct phases are possible if and only if $n$ jumps by 1 .

Can such rules be violated in the presence of interactions? The classification of the gapped phases can itself, of course, be changed by interactions, but here we are interested in the phenomenon of band-theory-forbidden quantum criticality between band-allowed phases. For the standard integer quantum Hall system discussed above, it is not known to us if the rule $\Delta n=1$ survives the inclusion of interactions. However, a closely related system provides us with a simple example where interactions modify an analogous band-theory rule.

Consider a system with two species of fermionsdenoted $\psi$ and $\chi$-in two space dimensions. We assume that there is a global $U(1)$ symmetry under which $\psi$ has charge 1 and $\chi$ is neutral. Within free fermion theory, gapped ground states of this system are now characterized by a pair of integers $(n, m)$. The electrical Hall conductivity is $\sigma_{x y}=n$, while the thermal Hall conductivity is $\kappa_{x y}=\bar{c}\left[\left(\pi^{2} k_{B}^{2} T\right) / 3\right]$, with $\bar{c}=(m / 2)$. Compared to the standard integer quantum Hall system, the presence of the additional neutral fermion means that $\bar{c}$ can take any multiple of half-integer value and is not tied to $\sigma_{x y}$. Within free fermion theory, a generic continuous transition between these phases satisfies the following rules: (i) $\Delta n=1, \Delta m=2$ or (ii) $\Delta n=0, \Delta m=1$. The former can be understood as a quantum Hall transition of the $\psi$ fermion, and the latter as a transition of the $\chi$ fermion.

Now, we show that this rule can be violated in the presence of short-ranged interactions that preserve the global $U(1)$ symmetry. Imagine an interaction such that the charged fermion forms a three-body bound state (a "cluston" [157]), $\psi_{3} \sim \psi \psi \psi \psi$. A cluston integer quantum Hall state [157] is then clearly possible with $\sigma_{x y}=9 k$, and $\bar{c}=k$ with $k \in \mathbb{Z}$. In this system, where both charged and neutral fermions are present, such a cluston integer quantum Hall state can also be accessed within free fermion theory: It corresponds to $n=9 k, m=2 k$. Now, consider a cluston integer quantum Hall transition, which can be second order so long as $\Delta k=1$. This case corresponds to $\Delta n=9, \Delta m=2$, which violates the band-theory rules discussed in the previous paragraph, even though both phases are band allowed. The critical theory has gapless clustons, but the $\psi, \chi$ particles are gapped.

\section{APPENDIX E: A 2 + 1-D BOSONIC MOTT INSULATOR TO $Z_{2}$ TOPOLOGICAL ORDER TRANSITION}

Here, we provide another example of a continuous phase transition in which modifying the properties of a gapped spectator field changes the nearby phase but not the universality class of the transition. We consider a transition from a $2+1-\mathrm{D}$ bosonic Mott insulator to a $Z_{2}$ topological order.

Consider a bosonic system in a Mott insulating phase. The physical bosons $b$ are gapped. We assume the system has a time-reversal symmetry $\mathcal{T}$, and the physical bosons are Kramer's singlets. Now, consider partons of the physical boson. We decompose the physical boson into two bosonic partons, which we call the $e$ particles. This fractionalization introduces a $Z_{2}$ gauge field, and the $e$ particles carry $Z_{2}$ gauge charge 1 . The $Z_{2}$ gauge field also has $\pi$-flux 
excitations, which we label as the $m$ particle. The $e$ and $m$ particles have mutual Berry's phase $\pi$. The Mott insulating phase is the confined phase of the $Z_{2}$ gauge field. The $Z_{2}$ confined phase can be viewed as a condensed phase of the $m$ particles.

Let us imagine that, by tuning some parameter, we can drive the system through a deconfinement transition to a $Z_{2}$ topological order. We can view the deconfinement transition as the proliferation of the vortices of the $m$-particle condensation. The transition is in the Ising universality class. After the transition, the $m$ particle is gapped, and the $Z_{2}$ gauge field is deconfined. The resultant phase has a $Z_{2}$ topological order. Throughout the transition, the $e$ particle remains gapped and does not participate in the low-energy theory. We can view them as the massive spectator fields in our system. Since the system has time-reversal symmetry, there are actually different classes of $Z_{2}$ topological orders distinguished by their time-reversal properties. These are called SET orders. In our case, the time-reversal properties of the spectator $e$ particle precisely determine which SET state we get for the deconfined phase. There are two choices. One is the $e$ particle, which is a Kramer's singlet. In this case, the resultant deconfined phase is a vanilla $Z_{2}$ topological order, which we can label as $e 0 m 0$, meaning that both $e$ and $m$ are Kramer's singlets. The other choice is that the $e$ particle actually carries a Kramer's doublet [158]. In this case, we get a nontrivial symmetry-enriched $Z_{2}$ topological order labeled by $e T m 0$. Note that $e T m 0$ and $e 0 m 0$ are distinct phases if the system preserves the timereversal symmetry. However, since the $e$ particle remains gapped during the transition, it cannot change the universality class of the transition.

\section{APPENDIX F: FRACTIONALIZING LOOPS}

Consider a quantum system with a Hilbert space of unoriented loops in 3+1-D. In this Appendix, we briefly describe phases of such a system where the loops have "fractionalized." Loop fractionalization played an important role in the topological order discussed in Sec. VI.

We can think of the system of unoriented loops as pure $Z_{2}$ gauge theory, i.e., without any matter. Formally, such a system has a global $Z_{2} 1$-form symmetry [denoted $\left(Z_{2}\right)_{1}$ ] associated with the unbreakable unoriented loops. There are some obvious phases of this loop system. First, there is a phase in which the loops have line tension, and there are no other excitations. This phase is the confined phase of the pure $Z_{2}$ gauge theory. The 1 -form $\left(Z_{2}\right)_{1}$ symmetry remains unbroken in this phase. Second, there is a distinct phase where the loops have zero line tension, and the pure $Z_{2}$ gauge theory is in its deconfined phase. Then, $\left(Z_{2}\right)_{1}$ is spontaneously broken.

Here, we are interested in a different kind of phase where the microscopic loops (denoted strength-1) are tensionful but have fractionalized into other loops. In other words, the 1-form global symmetry has been fractionalized.
We describe a simple example of such a loop-fractionalized phase where there are two kinds of excitations:

(1) A strength- $1 / 2$ loop with line tension. Two strength$1 / 2$ loops fuse into a single microscopic strength- 1 loop.

(2) A point-particle excitation that braids with the $\pi$ phase with the fractionalized strength- $1 / 2$ loop.

This excitation structure is that of an emergent deconfined $Z_{2}$ gauge theory (not to be confused with the microscopic pure $Z_{2}$ gauge theory). Let us explicitly construct this phase. To this end, we begin by first considering a $U(1)$ gauge theory with a $\left(Z_{2}\right)_{1}$ symmetry. This theory has a gapless photon, gapped electric charges $E$, gapped magnetic charges $M$, and their bound states. Now, assume that all particles with odd magnetic charge are thrown out of the $U(1)$ gauge theory. Then, odd strength magnetic loops cannot end, and there is an exact $\left(Z_{2}\right)_{1}$ symmetry. This symmetry is broken spontaneously in the $U(1)$ gauge theory (the odd strength magnetic loops are tensionless). Consider now a Higgs transition obtained by condensing the basic $E$ particle. All magnetic flux loops then have line tension, and we get the "trivial" phase of loops with unbroken $\left(Z_{2}\right)_{1}$. If instead we consider a Higgs transition obtained by condensing $E^{2}$ without condensing $E$, we get a $Z_{2}$ gauge theory where $E$ survives as the $Z_{2}$ gauge charge. We also get strength-1/2 magnetic flux loops with line tension, which braid with the $\pi$ phase with the $Z_{2}$ gauge charge. Of course, strength-1 magnetic loops also have line tension and cannot break. We identify them with the microscopic loops. This state preserves $\left(Z_{2}\right)_{1}$ and is exactly the loop-fractionalized phase described above [159].

An effective field theory for this loop-fractionalized phase is readily written down. Consider the Lagrangian

$$
\mathcal{L}=\frac{1}{\pi} \beta \wedge d \alpha+\frac{1}{2 \pi} B \wedge d \alpha,
$$

where $\alpha$ is a 1 -form dynamical gauge field, and $\beta$ is a 2 -form dynamical gauge field. Note that $B$ is a 2 -form background gauge field that couples to the global $\left(Z_{2}\right)_{1}$ symmetry. The first term is the standard "BF" theory description of $Z_{2}$ gauge theory. It dictates that the strings that are charged under $\beta$ are seen as the $\pi$ flux of the $\alpha$. These strings are the tensionful loops of the $Z_{2}$ gauge theory. The "microscopic" loops that couple to $B$, however, have $2 \pi$ flux of $\alpha$. Thus, this action correctly captures the loop-fractionalized phase described above.

\section{APPENDIX G: THE $S U(2)+N_{f}^{A}$ THEORY WITH $N_{f}^{A} \in 2 \mathbb{Z}$}

In this Appendix, we provide generalizations of the previous fermionic, deconfined, quantum critical points in Sec. IV. We extend the $S U(2)+N_{f}^{A}$ theories to even $N_{f}^{A}$ cases.

Let us consider an $S U(2)$ gauge theory coupled to $N_{f}^{A}=2$ flavors of adjoint Dirac fermions. The $3+1-\mathrm{D}$ Lagrangian of this theory is 
$\mathcal{L}_{A d j 2}^{S U(2)}=\sum_{i=1}^{2} i \bar{\psi}_{i} \gamma_{\mu}\left(\partial_{\mu}-a_{\mu}^{\alpha} T^{\alpha}\right) \psi_{i}-m \bar{\psi}_{i} \psi_{i}+\cdots$.

Analytically, this theory is expected to be inside the conformal window $[88,90]$. Numerically, it is found that the infrared limit for the $m=0$ theory is consistent with a conformal field theory [89]. We want to understand what phase transition this theory describes. To be more precise, we content ourselves with determining the topological distinction between the phases with the two signs of $m$, assuming large $|m|$. We do not attempt to answer the question of whether there are other intermediate phases at small $|m|$. Accordingly, whenever we talk about the massive theory below, we implicitly mean the theory at large $|m|$. If we tune $m$ to be nonzero, the fermions are gapped. As usual, we can regularize the theory such that, for $m<0$, integrating out the massive fermion generates zero $\Theta$ angle for the $S U(2)$ gauge theory, in which case the theory will enter a confined phase in the low-energy limit. For $m>0$, the massive fermions contribute an $8 \pi \Theta$ angle for the $S U(2)$ gauge fields. Since the $\Theta$ term is $2 \pi$ periodic, the $S U(2)$ gauge theory will again be confined in the infrared limit. The question is, what is the nature of the gapped phases for $m<0$ and $m>0$ ? The two states can only differ in their topological aspects. They can be different SPT states of certain global symmetry.

For general mass $m$, the global symmetry of the theory is $\mathcal{G}=S O(4) \times Z_{2}^{T}$. The time-reversal symmetry transformation is, as usual,

$$
Z_{2}^{T}: \psi \rightarrow \gamma_{0} \gamma_{5} \psi^{\dagger}
$$

where we suppress the flavor and gauge indices. To see the $S O(4)$ symmetry, we decompose the two flavors of Dirac fermions into four flavors of Majorana fermions. The $S O(4)$ symmetry is then a flavor rotation between the four Majorana fermions. Since the $S U(2)$ adjoint representation is a real representation, the $S O(4) \times Z_{2}^{T}$ symmetry commutes with the gauge group. This symmetry is not anomalous, and it is an exact symmetry for any $m$.

Let us first discuss the classification of interacting fermion SPT with $S O(4) \times Z_{2}^{T}$ symmetry. In the free fermion limit, the $3+1$-D fermion SPT classification is $\mathbb{Z}$. The root state for this class is four copies of topological superconductors with $Z_{2}^{T}$ symmetry (DIII class), where the $S O(4)$ rotates among the four copies. The typical surface theory of such a root state is four copies of gapless Majorana fermions. In the free fermion limit, since the DIII class is $\mathbb{Z}$ classified, the classification of $S O(4) \times Z_{2}^{T}$ SPT is $\mathbb{Z}$ as well. With an interaction, the classification becomes $\mathbb{Z}_{4} \times \mathbb{Z}_{2}$. The $\mathbb{Z}_{2}$ part corresponds to the pure $Z_{2}^{T}$ SPT state labeled by its anomalous surface $Z_{2}$ topological order ef $m f$, which only appears in the interacting system and has no free fermion correspondence. The free fermion $\mathbb{Z}$ classification is reduced to $\mathbb{Z}_{4}$ by the interaction (which is different from the odd $N_{f}$ case). The reason for this result is as follows. The pure time-reversal anomaly on the $2+1-\mathrm{D}$ surface is $\mathbb{Z}_{16}$ classified, which means that multiples of 16 copies of $2+1$-D Majorana fermions are time-reversal anomaly-free. Therefore, we need at least four copies of the root state to cancel the time-reversal anomaly on the surface. Next, we need to consider the mixed anomaly between $S O(4)$ and $Z_{2}^{T}$. This anomaly is related to the generalized parity anomaly. According to Ref. [128], by considering the system on a general unorientable manifold, the surface theory of four copies of the root states will be free from the mixed anomaly between $S O(4)$ and $Z_{2}^{T}$ symmetry. Physically, this means that the $S O(4)$ monopole in the $3+1$-D bulk carries a trivial time-reversal quantum number. Combining the two constraints, we conclude that the interaction classification reduced from the free fermion states is $\mathbb{Z}_{4}$. We can also see this from a surface argument. Let us take four copies of the root states. The boundary theory consists of 16 copies of Majorana fermions. The 16 Majorana fermions form four copies of vector representations under $S O(4)$. The question is whether we can gap them out while preserving the $S O(4) \times Z_{2}^{T}$ symmetry. We can group the 16 Majorana fermions into eight Dirac fermions and assume there is an extra $U(1)_{e}$ symmetry for the Dirac fermions, which we will eventually explicitly break. The Dirac fermion has two indices, an $S O(4)$ vector index $v=1,2,3,4$ and another flavor index $i=1,2$.

$$
H_{\times 4}=\sum_{i=1}^{2} \sum_{v=1}^{4} \psi_{i, v}^{\dagger}\left(i \partial_{x} \sigma^{x}+i \partial_{y} \sigma^{z}\right) \psi_{i, v} .
$$

The symmetry transformations are

$$
\begin{gathered}
U(1)_{e}: \psi_{i, v} \rightarrow e^{i \theta} \psi_{i, v}, \\
Z_{2}^{T}: \psi_{i, v} \rightarrow i \sigma^{y} \psi_{i, v}^{\dagger}, \\
S O(4): \psi_{i, v} \rightarrow O_{v, w} \psi_{i, w}, \quad O \in S O(4) .
\end{gathered}
$$

Now, we introduce a superconducting order parameter just as in Eq. (61). This term breaks both $U(1)_{e}$ and $Z_{2}^{T}$ but preserves a combination of $Z_{2}^{T}$ and $U(\pi / 2)$ rotation. Consider the $\pi$ vortex of the superconductor order parameter. It carries eight Majorana zero modes labeled by $\chi_{i, v}$. We can combine them into four complex zero modes, $f_{v}=\chi_{1, v}+i \chi_{2, v}$. We can write down an $S O(4)$ invariant four-fermion interaction of the form $H_{\text {int }}=$ $-V\left(f_{1}^{\dagger} f_{2}^{\dagger} f_{3}^{\dagger} f_{4}^{\dagger}+\right.$ H.c. $)$ [118]. This interaction leads to an $S O(4)$ symmetric ground state $\left|\psi_{v}\right\rangle=\left(|0\rangle+f_{1}^{\dagger} f_{2}^{\dagger} f_{3}^{\dagger} f_{4}^{\dagger}|0\rangle\right) /$ $\sqrt{2}$ and a gapped spectrum for the vortex core. Now, we can condense the $\pi$ vortices and restore the $U(1)_{e}$ and $Z_{2}^{T}$ symmetry. The resultant surface state is a trivial gapped 
symmetric state under $U(1)_{e} \times S O(4) \times Z_{2}^{T}$ symmetry. We can then turn on a small explicit $U(1)_{e}$ breaking term. Since the surface is now trivially gapped, it is stable against any small perturbation. Thus, we proved that the surface of four copies of the root states can be trivially gapped while preserving the $S O(4) \times Z_{2}^{T}$ symmetry, which is equivalent to saying that the bulk state is topologically trivial.

Next, we want to determine which SPT state the $m>0$ phase falls into. We can always regularize the system such that the $m<0$ phase is the trivial class of the SPT states under this global symmetry. To detect the topological properties of the $m>0$ phase, we can derive the topological response for the background $S O(4)$ gauge field on an orientable manifold,

$$
\begin{aligned}
\mathcal{S}_{\text {topo }} & =i \frac{\pi}{2}\left(p_{1}\left(A^{S O(12)}\right)-\frac{12}{8} \sigma\right) \\
& =i \frac{\pi}{2}\left(3 p_{1}\left(A^{S O(4)}\right)+4 p_{1}\left(a^{S O(3)}\right)-\frac{6}{4} \sigma\right) \\
& =i 3 \pi\left(\frac{1}{2} p_{1}\left(A^{S O(4)}\right)-\frac{1}{4} \sigma\right)+i 2 \pi p_{1}\left(a^{S O(3)}\right) \\
& =i 3 \pi\left(S_{\Theta}^{S O(4)}-\frac{x}{4} \sigma\right) .
\end{aligned}
$$

This nontrivial response theory tells us that the $m>0$ state is indeed a nontrivial SPT protected by the $S O(4) \times Z_{2}^{T}$ symmetry.

As before, to understand the theory, we need to introduce the spin- $1 / 2$ spectator field. Let us take the simplest case where the spectator is a scalar under $S O(4)$ and a singlet under $Z_{2}^{T}$ as in Eq. (69). In this case, we can do similar surface analysis as in the previous sections to understand the $m>0$ phase. The natural surface state of the $m>0$ system is $S U(2) \mathrm{QCD}_{3}$, with two flavors of adjoint massless Dirac fermions. We can condense the trivial spectator boson to Higgs out the $S U(2)$ gauge field. The surface state results in six physical Dirac fermions or 12 physical Majorana fermions with identical time-reversalsymmetry transformation. This state corresponds to $n=$ $3 \sim-1$ in the $\mathbb{Z}_{4}$ classification.

Now, if the spectator is a scalar under $S O(4)$ and a Kramers doublet under $Z_{2}^{T}$ transforming as in Eq. (76), we can run a similar argument as in Sec. IV. Condensation of the spectator field will not break the physical time-reversal symmetry. The system will be invariant under a gaugeequivalent time-reversal transformation $\tilde{Z}_{2}^{T}$ as in Eq. (77). Since the gauge transformations also change the timereversal transformation on the adjoint fermions as in Eq. (78), the resulting state is now $n=-2+1=-1 \sim 3$ in the $\mathbb{Z}_{4}$ classification.

Notice that, in this case, the topological index of the $m>0$ phase actually does not depend on the two choices of the spectator fields. This case is indeed consistent with the bulk analysis. We show that, in this case, the neutral $S O$ (3) monopole in the bulk is a Kramers singlet boson. Therefore, the two choices of the spectator fields do not have different surface time-reversal anomalies. For the zero modes in the $S O(3)$ monopole, we consider the system with a spherical geometry and set the background $S O(3)$ gauge field such that there is $2 \pi$ magnetic flux coming out of the sphere along the $z$ direction in the flavor space. For the $m>0$ phase, the surface theory hosts gapless Dirac fermions, which contribute zero modes for the monopole configuration. Let us write down the surface state:

$$
\begin{aligned}
\mathcal{L}_{\text {surf }}^{N_{f}^{A}=2}= & \sum_{i=1}^{2} i \bar{\psi}_{+, i} \gamma_{\mu}\left(\partial_{\mu}-i a_{\mu}^{z}\right) \psi_{+, i} \\
& +\sum_{i=1}^{2} i \bar{\psi}_{-, i} \gamma_{\mu}\left(\partial_{\mu}+i a_{\mu}^{z}\right) \psi_{-, i} \\
& +\sum_{i=1}^{2} i \bar{\psi}_{0, i} \gamma_{\mu} \partial_{\mu} \psi_{0, i} .
\end{aligned}
$$

Here, we see that there are three classes of Dirac fermions that carry \pm 1 and 0 gauge charge under $a_{\mu}^{z}$, respectively. Each class has two flavors. For each class, we can decompose the two Dirac fermions into four Majorana fermions, and they form a vector representation of the global $S O(4)$ symmetry. Now, let us consider the zero modes in the $2 \pi$ flux. There are, in total, four complex zero modes labeled by $f_{+, 1}, f_{+, 2}, f_{-, 1}, f_{-, 2}$. The \pm denote the gauge charge that they carry. The Hilbert space spanned by these zero modes has 16 states. It is very easy to spot which states are gauge invariant but a little difficult to construct an $S O(4)$ scalar. To start, let us consider the four states constructed from $f_{+, 1}$ and $f_{+, 2}$. They can be group into two classes: $\left\{|0\rangle, f_{+, 1}^{\dagger} f_{+, 2}^{\dagger}|0\rangle\right\}$ and $\left\{f_{+, 1}^{\dagger}|0\rangle, f_{+, 2}^{\dagger}|0\rangle\right\}$. These two sets of states form the left- and right-handed spinor representations of the $S O(4)$ group [160]. We know that, for $S O(4)$, two left or two right spinors can be combined into an $S O(4)$ scalar [161]. Therefore, combining two leftor right-handed spinors from the $f_{+}$sector and the $f_{-}$sector, we can form such a gauge neutral and $S O(4)$ singlet state, for example, $\left(f_{+, 1}^{\dagger} f_{-, 2}^{\dagger}-f_{+, 2}^{\dagger} f_{-, 1}^{\dagger}\right)|0\rangle$. Under time-reversal transformation, $Z_{2}^{T}:|0\rangle \rightarrow f_{+, 1}^{\dagger} f_{+, 2}^{\dagger} f_{-, 1}^{\dagger} f_{-, 2}^{\dagger}|0\rangle, f_{ \pm, i} \rightarrow \mp i f_{ \pm, i}^{\dagger}$, this state returns to its original form. Therefore, the gauge and global neutral $S O(3)$ monopole is a Kramers singlet boson. This result corresponds to a trivial $m$ particle for the surface $Z_{2}$ topological order, which indicates that it is not anomalous. Hence, the two spectator choices make no difference on the topological index of the $m>0$ phase.

The above analysis also suggests a possible duality between the $3+1$-D $S U(2)+N_{f}^{A}=2$ theory and two free Dirac fermions with $S O(4) \times Z_{2}^{T}$ symmetry, as they both describe the continuous phase transition between the $n=0$ and $n=-1$ SPT states in this symmetry class. 
However, we will leave to a future study an analysis of the emergent symmetries and anomalies of the gauge theory.

For even $N_{f}^{A}>2$, the global symmetry of the system is $S O\left(2 N_{f}^{A}\right) \times Z_{2}^{T}$, and the interacting fermionic SPT classification is the same as the $N_{f}^{A}=2$ case. The $S U(2)+N_{f}^{A}$ theory is also a theory of a quantum phase transition between $n=0$ and $n=-1$ SPT states in this symmetry class. However, in this case, the gauge theory is free in the infrared limit. Therefore, we can tell with confidence that it is distinct from the phase transition theory in the free fermion setting. Thus, these theories provide other examples of multiple universality classes for the same phase transition.

\section{APPENDIX H: THE $S U(2)+N_{f}^{A}$ THEORY WITH $N_{f}^{A} \in \mathbb{Z}+\frac{1}{2}$}

The reason for $N_{f}^{A}$ being half-integer is that we consider, instead of Dirac fermions, Majorana fermions. Since the adjoint representation of $S U(2)$ is a real representation, we can easily generalize the theory to Majorana fermions. We thus consider $2 N_{f}^{A}=2 k+1(k \in \mathbb{Z})$ flavors of $S U(2)$ adjoint Majorana fermions whose $3+1-\mathrm{D}$ action can be written as

$\mathcal{L}_{\text {AdjMaj }}^{S U(2)}=\sum_{i=1}^{2 k+1} i \chi_{i}^{T} \gamma_{\mu}\left(\partial_{\mu}-a_{\mu}^{\alpha} T^{\alpha}\right) \chi_{i}-m \bar{\chi}_{i} \chi_{i}+\cdots$

(We still assume massive spin- $1 / 2$ spectator fields in the spectrum of our system.) The massless theory with $N_{f}^{A}=\frac{3}{2}$ or $k=1$ is inside the conformal window of adjoint $S U(2)$ gauge theory. For $N_{f}^{A}>2$ or $k>2$, the massless theory flows to the free fixed point in the infrared.

Let us first discuss the dynamical properties of the massive phase. As before, the $m<0$ phase can be regularized to have a trivial $\Theta$ angle for the $S U(2)$ gauge theory, and it enters a confined phase at low energy. On the $m>0$ side, the $\Theta$ angle for $S U(2)$ is $4 k \pi+2 \pi$, which is also trivial because it is a multiple of $2 \pi$. Therefore, the $m>0$ side also enters a confined phase. As in all the other examples before, the two phases are not distinguished by their dynamical properties but by their topological properties.

The global symmetry in this system is $S O(2 k+1) \times Z_{2}^{T}$. The fermion SPT classification for this symmetry is $\mathbb{Z}_{16} \times \mathbb{Z}_{2}$. The $\mathbb{Z}_{2}$ part is the efmf state protected by $Z_{2}^{T}$ only. The $\mathbb{Z}_{16}$ part descends from the free fermion classification. The root state includes $2 k+1$ copies of the topological superconductor in the DIII class. The $2 k+1$ copies form a vector representation of $S O(2 k+1)$. Since the time-reversal anomaly for the DIII class is $\mathbb{Z}_{16}$ fold and $2 k+1$ is coprime with 16 , we need at least 16 root states to cancel the time-reversal anomaly on the surface. For 16 copies of the root states, there is also no mixed anomaly between $S O(2 k+1)$ and $Z_{2}^{T}$. (Using the argument in the previous section, the mixed anomaly is fourfold periodic.) Therefore, 16 is the minimal number of copies of the root states for an anomaly-free surface. Hence, the interactionreduced classification is $\mathbb{Z}_{16}$.

Let us now discuss the nature of the $m>0$ phase. We can derive the topological response theory for the background $S O(2 k+1)$ gauge field on the $m>0$ side on an orientable manifold,

$$
\begin{aligned}
\mathcal{S}_{\text {topo }}= & i \frac{\pi}{2}\left(p_{1}\left(A^{S O(3(2 k+1))}\right)-\frac{3(2 k+1)}{8} \sigma\right) \\
= & i \frac{\pi}{2}\left(3 p_{1}\left(A^{S O(2 k+1)}\right)+(2 k+1) p_{1}\left(a^{S O(3)}\right)\right. \\
& \left.-\frac{3(2 k+1)}{8} \sigma\right) \\
= & i 3 \pi\left(\frac{1}{2} p_{1}\left(A^{S O(2 k+1)}\right)-\frac{2 k+1}{16} \sigma\right) \\
& +i \pi(4 k+2) p_{1}\left(a^{S U(2)}\right) \\
= & i 3 \pi\left(S_{\Theta}^{S O(2 k+1)}-\frac{2 k+1}{16} \sigma\right) .
\end{aligned}
$$

Here, we use the fact that for both choices of spectator field on an orientable manifold, the gauge bundle must satisfy $w_{2}(S O(3))=0 \bmod 2$, which means the gauge bundle is a pure $S U(2)$ bundle. This response theory, while not revealing all the information about the $m>0$ phase, does tell us that the $m>0$ phase is topologically nontrivial. We still need to determine in which SPT the $m>0$ phase is.

We find that the nature of the $m>0$ phase depends on the properties of the spectator field. Assuming a spectator boson that is a $S O(2 k+1)$ scalar and time-reversal singlet as in Eq. (69), the topological index for the $m>0$ phase is the $n=3$ state in the $\mathbb{Z}_{16}$ classification. For the other case of a time-reversal doublet spectator as in Eq. (76), the topological index is $n=-1$. The arguments for these results are straightforward generalizations of surface arguments in Sec. IV. We note that the difference between the two cases is the $n=4$ state, which is not the $e \operatorname{Tm} T$ state in this situation. (The $e T m T$ state would correspond to the $n=8$ state in the $\mathbb{Z}_{16}$ classification.)

The time-reversal singlet spectator case gives us another example of a band-theory-forbidden continuous transition between band-theory-allowed insulating states. For the time-reversal doublet spectator case, with $k=1$ or $N_{f}^{A}=\frac{3}{2}$, the massless $S U(2)+N_{f}^{A}=\frac{3}{2}$ theory is a strongly coupled conformal field theory in the gauge-theory description. For $k>1$ or $N_{f}^{A}>2$, the massless $S U(2)+N_{f}^{A}$ theory is free in the infrared. This theory is clearly different from $2 k+1$ free massless Majorana fermions. However, both theories describe the same $n=0$ to $n=-1$ transition. 
TABLE I. A summary for $S U(2)+N_{f}^{A}$ theory with general $N_{f}^{A}$. The global symmetry associated with the system is $S O\left(2 N_{f}^{A}\right) \times Z_{2}^{T}$. The SPT classification depends on the $N_{f}^{A}$. The last two rows show the topological index for the $m>0$ phase for both choices of the spectator field. In all cases, $\eta=0$, so we do not include it in the table.

\begin{tabular}{lccc}
\hline \hline$N_{f}^{A}$ & $\mathbb{Z}+\frac{1}{2}$ & $2 \mathbb{Z}+1$ & $2 \mathbb{Z}$ \\
\hline Symmetry $\mathcal{G}$ & $S O(2 \mathbb{Z}+1) \times Z_{2}^{T}$ & $S O(4 \mathbb{Z}+2) \times Z_{2}^{T}$ & $S O(4 \mathbb{Z}) \times Z_{2}^{T}$ \\
fSPT classification & $\mathbb{Z}_{16} \times \mathbb{Z}_{2}$ & $\mathbb{Z}_{8} \times \mathbb{Z}_{2}$ & $\mathbb{Z}_{4} \times \mathbb{Z}_{2}$ \\
$\mathcal{T}^{2}=1$ spectator & $n=3$ & $n=3$ & $n=3 \sim-1$ \\
$\mathcal{T}^{2}=-1$ spectator & $n=-1$ & $n=-1$ & $n=-1 \sim 3$ \\
\hline \hline
\end{tabular}

Therefore, this provides more examples for multiversality classes.

A summary of all the results for the three $N_{f}^{A}$ series is tabulated in Table I.

\section{APPENDIX I: AN $S U(4)$ GENERALIZATION}

In this Appendix, we explore a generalization with a different gauge group. To make things simple, let us restrict our attention to only one flavor of adjoint Dirac fermion. Let us consider $3+1-\mathrm{D} S U(4)$ gauge theory coupled to one flavor of adjoint fermion. The adjoint Dirac fermion has 15 components. The Lagrangian is written as follows:

$$
\mathcal{L}_{\text {adj }}^{S U(4)}=i \bar{\psi} \gamma_{\mu}\left(\partial_{\mu}-a_{\mu}^{a} T^{a}\right) \psi-m \bar{\psi} \psi+\cdots,
$$

where $T^{a}$ 's, the generators of the $S U(4)$ group, are $15 \times 15$ matrices. The infrared limit of the massless theory is still unclear. Let us assume it is inside the conformal window for the moment.

We first consider the dynamical properties of the massive phases. For $m<0$, the fermions are massive, and we can integrate them out. We choose a regularization such that the $\Theta$ angle for the $S U(4)$ gauge theory is 0 . The $S U(4)$ gauge theory enters a confined phase at low energy. With this regularization, we can calculate the $\Theta$ angle of the $S U(4)$ gauge theory for the $m>0$ phase as follows [162]:

$$
\mathcal{L}_{\Theta}^{S U(4)}=\frac{\pi}{2} \operatorname{Tr}_{A} \frac{F^{A}}{2 \pi} \wedge \frac{F^{A}}{2 \pi}=8 \pi\left(\frac{1}{2} \operatorname{Tr}_{f} \frac{F^{f}}{2 \pi} \wedge \frac{F^{f}}{2 \pi}\right) .
$$

The $\Theta$ angle is $8 \pi$, which is equivalent to being trivial because of the $2 \pi$ periodicity. Therefore, the $S U(4)$ gauge theory on the $m>0$ side is also confined. Next, we discuss the topological difference between the two massive phases.

First, let us identify the symmetries. The 0 -form global symmetry of the theory is $U(1) \times Z_{2}^{T}$ [163]. The time reversal and $U(1)$ transformation are the same as the AIII class in Eqs. (81) and (82). The global symmetry commutes with the $S U(4)$ gauge group. We also assume a massive bosonic spectator $z$ that carries an $S U(4)$ fundamental representation. This breaks the 1 -form $Z_{4}$ center symmetry in the system. There are clearly gauge-invariant fermions in the system such as $\left(z^{\dagger} T^{a} z\right) \psi^{a}$. Therefore, the massless theory describes a critical point in a fermionic system.

Let us consider the case where the spectator is neutral under global $U(1)$ and a singlet under time-reversal transformation:

$$
U(1): z \rightarrow z ; \quad Z_{2}^{T}: z \rightarrow z^{*} .
$$

We note that the $\mathcal{T}^{2}= \pm 1$ is meaningless in this case for the spectator boson. We can redefine the time-reversal transformation to be $\tilde{Z}_{2}^{T}: z \rightarrow e^{i \pi / 2} z^{*}$, where the phase rotation is an element of the center of the $S U(4)$ gauge group. This gauge-equivalent time reversal has $\mathcal{T}^{2}=-1$ for the spectator boson. We also notice that the adjoint fermion has identical time-reversal transformation for $\tilde{Z}_{2}^{T}$ and $Z_{2}^{T}$.

Let us regularize the $m<0$ phase such that it is in the topologically trivial state. Then, consider the $m>0$ phase. We again consider the surface state of the system to determine the topological properties of the system. The natural surface state of the system is 2+1-D QCD of the $S U(4)$ gauge theory coupled to one adjoint fermion. On the surface, we can condense the spectator field, which Higgses the $S U(4)$ gauge field completely while preserving the $U(1) \times Z_{2}^{T}$ symmetry. The 15 Dirac fermions in the $S U(4)$ adjoint fermion become physical fermions with identical $U(1) \times Z_{2}^{T}$ transformations. Therefore, this state has a topological index in the AIII class, $n=15 \sim-1$. Thus, in the large-mass limit, we either get a trivial insulator or the simplest topological superconductor. Study of the smallmass limit within this framework may reveal interesting possible evolutions between these two familiar phases. However, we will leave this to future work.

\section{APPENDIX J: LOCAL OPERATORS IN THE ADJOINT $S U(2)$ GAUGE THEORY}

In this Appendix, we list the gauge-invariant operators of the $S U(2)$ gauge theory with one adjoint Dirac fermion (the theory discussed in Sec. V). We organize these local operators according to their quantum numbers under the Lorentz group and the emergent global symmetry group. We only list Lorentz scalars (Table II) and spinors (Table III) composed of the adjoint fermions $\psi^{a}$ and gluon 
TABLE II. A summary of Lorentz scalars.

\begin{tabular}{lcccc}
\hline \hline Operator $\mathcal{O}$ & Weyl Rep & $U(1)_{c}$ & $U(1)_{A}$ & $Z_{2}^{T}$ (or $\mathcal{C}$ ) \\
\hline $\bar{\psi}^{a} \psi^{a}$ & $\xi_{1}^{a \dagger} \epsilon \xi_{2}^{a *}-\xi_{2}^{a T} \epsilon \xi_{1}^{a}$ & 0 & $\ldots$ & $\mathcal{O} \rightarrow \mathcal{O}$ \\
$\bar{\psi}^{a} i \gamma_{5} \psi^{a}$ & $i \xi_{1}^{a \dagger} \epsilon \xi_{2}^{a *}+i \xi_{2}^{a T} \epsilon \xi_{1}^{a}$ & 0 & $\ldots$ & $\mathcal{O} \rightarrow-\mathcal{O}$ \\
$i \bar{\psi} \bar{\psi}^{a} \psi^{a}+\bar{\psi}^{a} i \gamma_{5} \psi^{a}$ & $2 i \xi_{1}^{a \dagger} \epsilon \xi_{2}^{a *}$ & 0 & -2 & $\mathcal{O} \rightarrow-\mathcal{O}$ \\
$-i \bar{\psi}^{a} \psi^{a}+\bar{\psi}^{a} i \gamma_{5} \psi^{a}$ & $2 i \xi_{2}^{a T} \epsilon \xi_{1}^{a}$ & 0 & +2 & $\mathcal{O} \rightarrow-\mathcal{O}$ \\
$\psi^{a T} C \psi^{a}$ & $-\xi_{1}^{a T} \epsilon \xi_{1}^{a}+\xi_{2}^{a \dagger} \epsilon \xi_{2}^{a *}$ & 2 & $\ldots$ & $\mathcal{O} \rightarrow \mathcal{O}^{\dagger}$ \\
$\psi^{a T} C \gamma_{5} \psi^{a}$ & $-\xi_{1}^{a T} \epsilon \xi_{1}^{a}-\xi_{2}^{a \dagger} \epsilon \xi_{2}^{a *}$ & 2 & $\ldots$ & $\mathcal{O} \rightarrow-\mathcal{O}^{\dagger}$ \\
$\mathcal{O}_{+2} \sim \psi^{a T} C \psi^{a}+\psi^{a T} C \gamma_{5} \psi^{a}$ & $-2 \xi_{1}^{a T} \epsilon \xi_{1}^{a}$ & 2 & 2 & $\mathcal{O}_{+2} \rightarrow \mathcal{O}_{-2}$ \\
$\mathcal{O}_{-2} \sim\left(\psi^{a T} C \psi^{a}\right)^{\dagger}-\left(\psi^{a T} C \gamma_{5} \psi^{a}\right)^{\dagger}$ & $-2 \xi_{2}^{a T} \epsilon \xi_{2}^{a}$ & -2 & 2 & $\mathcal{O}-\mathcal{O}_{+2}$ \\
$\epsilon_{\mu \nu \lambda \rho} F_{\mu \nu}^{a} F_{\lambda \rho}^{a}$ & $\ldots$ & 0 & 0 & $\mathcal{O} \rightarrow-\mathcal{O}$ \\
$\epsilon_{a b c} F_{\mu \nu}^{a} F_{\nu \rho}^{b} F_{\rho \mu}^{c}$ & $\ldots$ & 0 & 0 & $\mathcal{O} \rightarrow \mathcal{O}$ \\
\hline \hline
\end{tabular}

TABLE III. A summary of Lorentz spinors.

\begin{tabular}{lcccc}
\hline \hline Operator & Weyl Rep & $U(1)_{c}$ & $U(1)_{A}$ & $Z_{2}^{T}$ (or $\left.\mathcal{C T}\right)$ \\
\hline$f_{\gamma_{0}} \sim \epsilon_{a b c}\left(\bar{\psi}^{a} \psi^{b}\right) \psi^{c}$ & $\ldots$ & 1 & $\ldots$ & $f_{\gamma_{0}} \rightarrow \gamma_{0} \gamma_{5} f_{\gamma_{0}}^{\dagger}$ \\
$f_{\gamma_{5}} \sim \epsilon_{a b c}\left(\bar{\psi}^{a} i \gamma_{5} \psi^{b}\right) \psi^{c}$ & $\ldots$ & 1 & $\ldots$ & $f_{\gamma_{5}} \rightarrow-\gamma_{0} \gamma_{5} f_{\gamma_{5}}^{\dagger}$ \\
$f_{+3} \sim \epsilon_{a b c}\left(\left(i \bar{\psi}^{a} \psi^{b}\right) \gamma_{5}+\left(\bar{\psi}^{a} i \gamma_{5} \psi^{b}\right)\right) \psi^{c}$ & $\eta_{\lambda} \sim(-1)^{\lambda} \epsilon_{a b c} i\left(\xi_{1}^{a} \epsilon \xi_{2}^{b}\right) \xi_{\lambda}^{c}$ & 1 & 3 & $f_{+3} \rightarrow-\gamma_{0} \gamma_{5} f_{+3}^{\dagger}$ \\
$f_{-1} \sim \epsilon_{a b c}\left(-\left(i \bar{\psi}^{a} \psi^{b}\right) \gamma_{5}+\left(\bar{\psi}^{a} i \gamma_{5} \psi^{b}\right)\right) \psi^{c}$ & $\eta_{\lambda} \sim(-1)^{\lambda} \epsilon_{a b c} i\left(\xi_{2}^{a \dagger} \epsilon \xi_{1}^{b *}\right) \xi_{\lambda}^{c}$ & 1 & -1 & $f_{-1} \rightarrow-\gamma_{0} \gamma_{5} f_{-1}^{\dagger}$ \\
$f_{g} \sim \sigma_{\mu \nu} F_{\mu \nu}^{a} \psi^{a}$ & $\ldots$ & 1 & 1 & $f_{g} \rightarrow \gamma_{0} \gamma_{5} f_{g}^{\dagger}$ \\
$f_{g}^{\prime} \sim \epsilon_{\mu \nu \lambda \rho} \sigma_{\mu \nu} F_{\lambda \rho}^{a} \psi^{a}$ & $\ldots$ & 1 & 1 & $f_{g}^{\prime} \rightarrow-\gamma_{0} \gamma_{5} f_{g}^{\prime \dagger}$ \\
$f_{g g} \sim \epsilon_{a b c} F_{\mu \nu}^{a} F_{\mu \nu}^{b} \psi^{c}$ & $\ldots$ & 1 & 1 & $f_{g g} \rightarrow \gamma_{0} \gamma_{5} f_{g g}^{\dagger}$ \\
$f_{g g}^{\prime} \sim \epsilon_{a b c} \epsilon_{\mu \nu \lambda \rho} F_{\mu \nu}^{a} F_{\lambda \rho}^{b} \psi^{c}$ & $\ldots$ & 1 & 1 & $f_{g g}^{\prime} \rightarrow-\gamma_{0} \gamma_{5} f_{g g}^{\prime \dagger}$ \\
\hline \hline
\end{tabular}

fields $F_{\mu \nu}^{a}$ (up to the product of three operators). This list may be useful for future numerical studies to identify the low-energy Dirac fermion. As a reminder, the time-reversal transformations $(\mathcal{C T}$, to be more precise) on the Weyl fermions and the gluon fields are as follows:

$$
\begin{gathered}
\mathcal{C T}: \xi_{1} \rightarrow \epsilon \xi_{2}, \xi_{2} \rightarrow \epsilon \xi_{1} \quad \text { with } \quad \epsilon=i \sigma^{y}, \\
\mathcal{C T}: F_{\mu \nu}^{a} \rightarrow s_{\mu} s_{\nu} F_{\mu \nu}^{a} \quad \text { where } s_{\mu}=(+,-,-,-) .
\end{gathered}
$$

[1] S. Sachdev, Quantum Phase Transitions, 2nd ed. (Cambridge University Press, Cambridge, England, 2011).

[2] S. L. Sondhi, S. M. Girvin, J. P. Carini, and D. Shahar, Continuous Quantum Phase Transitions, Rev. Mod. Phys. 69, 315 (1997).

[3] C. Xu, Unconventional Quantum Critical Points, Int. J. Mod. Phys. B 26, 1230007 (2012).
[4] T. Senthil, A. Vishwanath, L. Balents, S. Sachdev, and M.P. A. Fisher, Deconfined Quantum Critical Points, Science 303, 1490 (2004).

[5] T. Senthil, L. Balents, S. Sachdev, A. Vishwanath, and M. P. A. Fisher, Quantum Criticality beyond the LandauGinzburg-Wilson Paradigm, Phys. Rev. B 70, 144407 (2004).

[6] T. Senthil, L. Balents, S. Sachdev, A. Vishwanath, and M. P. A. Fisher, Deconfined Criticality Critically Defined, J. Phys. Soc. Jpn. 74, 1 (2005).

[7] A. W. Sandvik, Evidence for Deconfined Quantum Criticality in a Two-Dimensional Heisenberg Model with FourSpin Interactions, Phys. Rev. Lett. 98, 227202 (2007).

[8] A. W. Sandvik, Continuous Quantum Phase Transition between an Antiferromagnet and a Valence-Bond Solid in Two Dimensions: Evidence for Logarithmic Corrections to Scaling, Phys. Rev. Lett. 104, 177201 (2010).

[9] R. G. Melko and R. K. Kaul, Scaling in the Fan of an Unconventional Quantum Critical Point, Phys. Rev. Lett. 100, 017203 (2008).

[10] K. Chen, Y. Huang, Y. Deng, A. B. Kuklov, N. V. Prokof'ev, and B. V. Svistunov, Deconfined Criticality Flow in the Heisenberg Model with Ring-Exchange Interactions, Phys. Rev. Lett. 110, 185701 (2013). 
[11] H. Shao, W. Guo, and A. W. Sandvik, Quantum Criticality with Two Length Scales, Science 352, 213 (2016).

[12] F.-J. Jiang, M. Nyfeler, S. Chandrasekharan, and U.-J. Wiese, From an Antiferromagnet to a Valence Bond Solid: Evidence for a First-Order Phase Transition, J. Stat. Mech. (2008) P02009.

[13] A. B. Kuklov, M. Matsumoto, N. V. Prokof'ev, B. V. Svistunov, and M. Troyer, Deconfined Criticality: Generic First-Order Transition in the SU(2) Symmetry Case, Phys. Rev. Lett. 101, 050405 (2008).

[14] L. Bartosch, Corrections to Scaling in the Critical Theory of Deconfined Criticality, Phys. Rev. B 88, 195140 (2013).

[15] G. J. Sreejith and S. Powell, Scaling Dimensions of Higher-Charge Monopoles at Deconfined Critical Points, Phys. Rev. B 92, 184413 (2015).

[16] A. W. Sandvik, S. Daul, R. R. P. Singh, and D. J. Scalapino, Striped Phase in a Quantum xy Model with Ring Exchange, Phys. Rev. Lett. 89, 247201 (2002).

[17] A. Sen and A. W. Sandvik, Example of a First-Order Néel to Valence-Bond-Solid Transition in Two Dimensions, Phys. Rev. B 82, 174428 (2010).

[18] A. Tanaka and X. Hu, Many-Body Spin Berry Phases Emerging from the $\pi$-Flux State: Competition between Antiferromagnetism and the Valence-Bond-Solid State, Phys. Rev. Lett. 95, 036402 (2005).

[19] C. Wang, A. Nahum, M. A. Metlitski, C. Xu, and T. Senthil, Deconfined Quantum Critical Points: Symmetries and Dualities, Phys. Rev. X 7, 031051 (2017).

[20] J. Lou, A. W. Sandvik, and N. Kawashima, Antiferromagnetic to Valence-Bond-Solid Transitions in Two-Dimensional $\mathrm{SU}(n)$ Heisenberg Models with Multispin Interactions, Phys. Rev. B 80, 180414(R) (2009).

[21] K. Harada, T. Suzuki, T. Okubo, H. Matsuo, J. Lou, H. Watanabe, S. Todo, and N. Kawashima, Possibility of Deconfined Criticality in $\mathrm{SU}(n)$ Heisenberg Models at Small n, Phys. Rev. B 88, 220408(R) (2013).

[22] A. Banerjee, K. Damle, and F. Alet, Impurity Spin Texture at a Deconfined Quantum Critical Point, Phys. Rev. B 82, 155139 (2010).

[23] A. Nahum, J. T. Chalker, P. Serna, M. Ortuño, and A. M. Somoza, Deconfined Quantum Criticality, Scaling Violations, and Classical Loop Models, Phys. Rev. X 5, 041048 (2015).

[24] A. Nahum, P. Serna, J. T. Chalker, M. Ortuño, and A. M. Somoza, Emergent SO(5) Symmetry at the Néel to Valence-Bond-Solid Transition, Phys. Rev. Lett. 115, 267203 (2015).

[25] G. Chen, J. Gukelberger, S. Trebst, F. Alet, and L. Balents, Coulomb Gas Transitions in Three-Dimensional Classical Dimer Models, Phys. Rev. B 80, 045112 (2009).

[26] D. Charrier, F. Alet, and P. Pujol, Gauge Theory Picture of an Ordering Transition in a Dimer Model, Phys. Rev. Lett. 101, 167205 (2008).

[27] D. Charrier and F. Alet, Phase Diagram of an Extended Classical Dimer Model, Phys. Rev. B 82, 014429 (2010).

[28] T. Senthil and M. P. A. Fisher, Competing Orders, Nonlinear Sigma Models, and Topological Terms in Quantum Magnets, Phys. Rev. B 74, 064405 (2006).
[29] A. Thomson and S. Sachdev, Fermionic Spinon Theory of Square Lattice Spin Liquids near the Néel State, Phys. Rev. X 8, 011012 (2018).

[30] X.-F. Zhang, Y.-C. He, S. Eggert, R. Moessner, and Frank Pollmann, Continuous Easy-Plane Deconfined Phase Transition on the Kagome Lattice, Phys. Rev. Lett. 120, 115702 (2018).

[31] C.-M. Jian, A. Thomson, A. Rasmussen, Z. Bi, and C. Xu, Deconfined Quantum Critical Point on the Triangular Lattice, Phys. Rev. B 97, 195115 (2018).

[32] L. Janssen and Y.-C. He, Critical Behavior of the $\mathrm{QED}_{3}$ Gross-Neveu Model: Duality and Deconfined Criticality, Phys. Rev. B 96, 205113 (2017).

[33] S. Pujari, K. Damle, and F. Alet, Néel-State to ValenceBond-Solid Transition on the Honeycomb Lattice: Evidence for Deconfined Criticality, Phys. Rev. Lett. 111, 087203 (2013).

[34] S. Pujari, F. Alet, and K. Damle, Transitions to ValenceBond Solid Order in a Honeycomb Lattice Antiferromagnet, Phys. Rev. B 91, 104411 (2015).

[35] A. Nahum, J. T. Chalker, P. Serna, M. Ortuño, and A. M. Somoza, 3D Loop Models and the $\mathrm{CP}^{n-1}$ Sigma Model, Phys. Rev. Lett. 107, 110601 (2011).

[36] S. D. Geraedts and O. I. Motrunich, Monte Carlo Study of a $u(1) \times u(1)$ System with $\pi$-Statistical Interaction, Phys. Rev. B 85, 045114 (2012).

[37] J. D'Emidio and R. K. Kaul, First-Order Superfluid to Valence-Bond Solid Phase Transitions in Easy-Plane $\mathrm{SU}(n)$ Magnets for Small n, Phys. Rev. B 93, 054406 (2016).

[38] J. D'Emidio and R. K. Kaul, New Easy-Plane $\mathbb{C P}^{N-1}$ Fixed Points, Phys. Rev. Lett. 118, 187202 (2017).

[39] R. K. Kaul, Spin Nematics, Valence-Bond Solids, and Spin Liquids in $\mathrm{SO}(n)$ Quantum Spin Models on the Triangular Lattice, Phys. Rev. Lett. 115, 157202 (2015).

[40] T. Grover and A. Vishwanath, Quantum Phase Transition between Integer Quantum Hall States of Bosons, Phys. Rev. B 87, 045129 (2013).

[41] Y.-M. Lu and D.-H. Lee, Quantum Phase Transitions between Bosonic Symmetry-Protected Topological Phases in Two Dimensions: Emergent $\mathrm{QED}_{3}$ and Anyon Superfluid, Phys. Rev. B 89, 195143 (2014).

[42] A. Vishwanath and T. Senthil, Physics of ThreeDimensional Bosonic Topological Insulators: SurfaceDeconfined Criticality and Quantized Magnetoelectric Effect, Phys. Rev. X 3, 011016 (2013).

[43] K. Slagle, Y.-Z. You, and C. Xu, Exotic Quantum Phase Transitions of Strongly Interacting Topological Insulators, Phys. Rev. B 91, 115121 (2015).

[44] Y.-Z. You, Z. Bi, D. Mao, and C. Xu, Quantum Phase Transitions between Bosonic Symmetry-Protected Topological States without Sign Problem: Nonlinear Sigma Model with a Topological Term, Phys. Rev. B 93, 125101 (2016).

[45] Y. Q. Qin, Y.-Y. He, Y.-Z. You, Z.-Y. Lu, A. Sen, A. W. Sandvik, C. Xu, and Z. Y. Meng, Duality between the Deconfined Quantum-Critical Point and the Bosonic Topological Transition, Phys. Rev. X 7, 031052 (2017).

[46] Y.-Y. He, H.-Q. Wu, Y.-Z. You, C. Xu, Z. Y. Meng, and Z.-Y. Lu, Bona Fide Interaction-Driven Topological 
Phase Transition in Correlated Symmetry-Protected Topological States, Phys. Rev. B 93, 115150 (2016).

[47] X. Chen, Z.-C. Gu, Z.-X. Liu, and X.-G. Wen, Symmetry Protected Topological Orders and the Group Cohomology of Their Symmetry Group, Phys. Rev. B 87, 155114 (2013).

[48] X. Chen, Z.-C. Gu, Z.-X. Liu, and X.-G. Wen, SymmetryProtected Topological Orders in Interacting Bosonic Systems, Science 338, 1604 (2012).

[49] A. Kitaev, Periodic Table for Topological Insulators and Superconductors, AIP Conf. Proc. 1134, 22 (2009).

[50] S. Ryu, A. P. Schnyder, A. Furusaki, and A. W. W. Ludwig, Topological Insulators and Superconductors: Tenfold Way and Dimensional Hierarchy, New J. Phys. 12, 065010 (2010).

[51] Z. Bi, A. Rasmussen, K. Slagle, and C. Xu, Classification and Description of Bosonic Symmetry Protected Topological Phases with Semiclassical Nonlinear Sigma Models, Phys. Rev. B 91, 134404 (2015).

[52] C. Wang, A. C. Potter, and T. Senthil, Classification of Interacting Electronic Topological Insulators in Three Dimensions, Science 343, 629 (2014).

[53] T. Senthil, Symmetry-Protected Topological Phases of Quantum Matter, Annu. Rev. Condens. Matter Phys. 6, 299 (2015), https://doi.org/10.1146/annurev-conmatphys031214-014740.

[54] P. Putrov, J. Wang, and S.-T. Yau, Braiding Statistics and Link Invariants of Bosonic/Fermionic Topological Quantum Matter in $2+1$ and $3+1$ Dimensions, Ann. Phys. (Amsterdam) 384, 254 (2017).

[55] N. Seiberg, T. Senthil, C. Wang, and E. Witten, A Duality Web in $2+1$ Dimensions and Condensed Matter Physics, Ann. Phys. (Amsterdam) 374, 395 (2016).

[56] C. Xu and Y.-Z. You, Self-Dual Quantum Electrodynamics as Boundary State of the Three-Dimensional Bosonic Topological Insulator, Phys. Rev. B 92, 220416(R) (2015).

[57] A. Karch and D. Tong, Particle-Vortex Duality from 3D Bosonization, Phys. Rev. X 6, 031043 (2016).

[58] P.-S. Hsin and N. Seiberg, Level/Rank Duality and ChernSimons-Matter Theories, J. High Energy Phys. 09 (2016) 095.

[59] C. Córdova and T. T. Dumitrescu, Candidate Phases for SU(2) Adjoint $\mathrm{QCD}_{4}$ with Two Flavors from $\mathcal{N}=2$ Supersymmetric Yang-Mills Theory, arXiv:1806.09592.

[60] M. M. Anber and E. Poppitz, Two-Flavor Adjoint QCD, Phys. Rev. D 98, 034026 (2018).

[61] J. Gomis, Z. Komargodski, and N. Seiberg, Phases of Adjoint $\mathrm{QCD}_{3}$ and Dualities, SciPost Phys. 5, 007 (2018).

[62] J. Terning, Modern Supersymmetry: Dynamics and Duality (Clarendon, Oxford, 2006), p. 324.

[63] M. E. Peskin, Mandelstam-'t Hooft Duality in Abelian Lattice Models, Ann. Phys. (N.Y.) 113, 122 (1978).

[64] C. Dasgupta and B. I. Halperin, Phase Transition in a Lattice Model of Superconductivity, Phys. Rev. Lett. 47, 1556 (1981).

[65] M. P. A. Fisher and D. H. Lee, Correspondence between Two-Dimensional Bosons and a Bulk Superconductor in a Magnetic Field, Phys. Rev. B 39, 2756 (1989).

[66] D. T. Son, Is the Composite Fermion a Dirac Particle?, Phys. Rev. X 5, 031027 (2015).
[67] C. Wang and T. Senthil, Dual Dirac Liquid on the Surface of the Electron Topological Insulator, Phys. Rev. X 5, 041031 (2015).

[68] M. A. Metlitski and A. Vishwanath, Particle-Vortex Duality of Two-Dimensional Dirac Fermion from ElectricMagnetic Duality of Three-Dimensional Topological Insulators, Phys. Rev. B 93, 245151 (2016).

[69] S. Jain, S. Minwalla, and S. Yokoyama, Chern Simons Duality with a Fundamental Boson and Fermion, J. High Energy Phys. 11 (2013) 037.

[70] G. Gur-Ari and R. Yacoby, Three Dimensional Bosonization from Supersymmetry, J. High Energy Phys. 11 (2015) 013.

[71] O. Aharony, Baryons, Monopoles and Dualities in ChernSimons-Matter Theories, J. High Energy Phys. 02 (2016) 093.

[72] K. Jensen, A Master Bosonization Duality, J. High Energy Phys. 01 (2018) 031.

[73] K. Jensen and A. Karch, Embedding Three-Dimensional Bosonization Dualities into String Theory, J. High Energy Phys. 12 (2017) 031.

[74] K. Jensen and A. Karch, Bosonizing Three-Dimensional Quiver Gauge Theories, J. High Energy Phys. 11 (2017) 018.

[75] A. Karch, B. Robinson, and D. Tong, More Abelian Dualities in $2+1$ Dimensions, J. High Energy Phys. 01 (2017) 017.

[76] A. Karch, D. Tong, and C. Turner, Mirror Symmetry and Bosonization in 2D and 3D, J. High Energy Phys. (2018) 059.

[77] K. Aitken, A. Baumgartner, and A. Karch, Novel 3D Bosonic Dualities from Bosonization and Holography, J. High Energy Phys. 09 (2018) 003.

[78] F. Benini, Three-Dimensional Dualities with Bosons and Fermions, J. High Energy Phys. 02 (2018) 068.

[79] D. Radicevic, D. Tong, and C. Turner, Non-Abelian 3D Bosonization and Quantum Hall States, J. High Energy Phys. 12 (2016) 067.

[80] Physically, this is a device that enables us to keep track of the fact that all physical fields with odd charge under $A$ are fermionic. Formally, if we try to formulate this theory on an arbitrary, compact, oriented space-time manifold, a $\operatorname{Spin}_{c}$ connection is like a $U(1)$ gauge field but with a modified flux quantization condition. Specifically, a $\operatorname{Spin}_{c}$ connection satisfies the following condition, $\int[F /(2 \pi)]=$ $\left(w_{2}^{T Y_{4}} / 2\right) \bmod 1$, where $F$ is the field strength for the $U(1)$ gauge bundle, $w_{2}^{T Y_{4}}$ is the second Stiefel-Whitney class for the tangent bundle [81], and the integral is taken over an arbitrary, oriented 2-cycle.

[81] M. Nakahara, Geometry, Topology and Physics (2003).

[82] This choice can always be made by suitable UV regulation of the theory.

[83] E. Witten, Fermion Path Integrals and Topological Phases, Rev. Mod. Phys. 88, 035001 (2016).

[84] This point of view is natural from a condensed matter perspective but may be unfamiliar to some high-energy theorists. We find it insightful to view the gauge theory this way. Note, in particular, that the fermionic matter fields, as well as the gauge fields themselves, should be regarded as emerging from these UV "local" bosons. 
[85] Throughout this paper, $N_{f}$ counts the number of Dirac fermions. In the literature, however, sometimes $N_{f}$ is used to denote the number of Weyl fermions.

[86] W.E. Caswell, Asymptotic Behavior of Non-Abelian Gauge Theories to Two-Loop Order, Phys. Rev. Lett. 33, 244 (1974).

[87] T. Banks and A. Zaks, On the Phase Structure of Vectorlike Gauge Theories with Massless Fermions, Nucl. Phys. B196, 189 (1982).

[88] T. Karavirta, J. Rantaharju, K. Rummukainen, and K. Tuominen, Determining the Conformal Window: SU(2) Gauge Theory with $n_{f}=4,6$ and 10 Fermion Flavours, J. High Energy Phys. 05 (2012) 003.

[89] A. Athenodorou, E. Bennett, G. Bergner, and B. Lucini, Infrared Regime of $S U(2)$ with One Adjoint Dirac Flavor, Phys. Rev. D 91, 114508 (2015).

[90] J. Rantaharju, T. Rantalaiho, K. Rummukainen, and K. Tuominen, Running Coupling in SU(2) Gauge Theory with Two Adjoint Fermions, Phys. Rev. D 93, 094509 (2016).

[91] T. A. Ryttov and R. Shrock, Higher-Loop Corrections to the Infrared Evolution of a Gauge Theory with Fermions, Phys. Rev. D 83, 056011 (2011).

[92] R. Shrock, Higher-Loop Structural Properties of the $\beta$ Function in Asymptotically Free Vectorial Gauge Theories, Phys. Rev. D 87, 105005 (2013).

[93] F. Sannino, Conformal Windows of $s p(2 n)$ and $s o(n)$ Gauge Theories, Phys. Rev. D 79, 096007 (2009).

[94] M. Mojaza, Aspects of Conformal Gauge Theories, Master Thesis, Syddansk University (2011).

[95] Our notation is $S p(1) \cong S U(2), \operatorname{PSp}(N)=S p(N) / Z_{2}$.

[96] The precise value of $y$ is readily determined by matching the RG flow for the gauge coupling at the $m=0$ fixed point with that of the pure gauge theory.

[97] In this case, we expect that since the fixed point appears at relatively weak coupling, introducing a nonzero bare mass will still drive the system to a confined phase. In other words, there is no intermediate phase that appears for small bare mass. This assumption is reasonable for theories in the conformal window that are "close" to the free fixed point. Later, when we consider the gauge theory with light adjoint fermions, we see that this assumption fails for theories far away from the perturbative regime.

[98] A formal but very useful description is to say that the $S U(2)$ gauge theory with only adjoint matter but no fundamental matter has a global $Z_{2}$ 1-form symmetry [denoted $\left(Z_{2}\right)_{1}$ ]. Of course, a microscopic condensed matter system of fermions has no such 1 -form symmetry. Therefore, we allow for an explicit breaking of the $\left(Z_{2}\right)_{1}$ symmetry by including the massive gauge spin- $1 / 2$ scalar.

[99] From a formal point of view, this corresponds to how to define the theory on nonorientable manifolds.

[100] C. Wang and T. Senthil, Interacting Fermionic Topological Insulators/Superconductors in Three Dimensions, Phys. Rev. B 89, 195124 (2014).

[101] Y.-Z. You and C. Xu, Symmetry-Protected Topological States of Interacting Fermions and Bosons, Phys. Rev. B 90, 245120 (2014).

[102] A somewhat similar duality in $2+1-\mathrm{D}$ was proposed [61] recently for $S U(2)$ gauge theory with $N_{f}=1 / 2$ adjoint fermions (i.e., with a single Majorana fermion in the adjoint representation). The IR theory was argued to consist of a free massless Majorana fermion augmented with a decoupled topological theory.

[103] Recently, the possibility of saturation of discrete mixed 1-form and 0-form anomalies by topological field theories through symmetry extension methods was discussed in Ref. [104]. Nonetheless, the most general criterions for such anomaly matching are not conclusive yet.

[104] Z. Wan and J. Wang, Adjoint $\mathrm{QCD}_{4}$, Deconfined Critical Phenomena, Symmetry-Enriched Topological Quantum Field Theory, and Higher Symmetry Extension, Phys. Rev. D 99, 065013 (2019).

[105] Z. Wan and J. Wang, Non-Abelian Gauge Theories, Sigma Models, Higher Anomalies, Symmetries, and Cobordisms, arXiv:1812.11967.

[106] Z. Wan and J. Wang, New Higher Anomalies, SU(N) YangMills Gauge Theory and $C P^{\mathrm{N}-1}$ Sigma Model, arXiv:1812.11968.

[107] From a formal point of view, despite the presence of fermionic matter fields, this theory can be defined on nonspin manifolds by choosing gauge bundles in $\left[S U(2)_{g} \times \operatorname{Spin}(4)\right] / Z_{2}$. On a nonspin manifold, we require $w_{2}\left(S O(3)_{g}\right)=w_{2}\left(T Y_{4}\right) \bmod 2$, where the left side is the second Stiefel-Whitney class of the $S O(3)$ gauge bundle and the right side is the second StiefelWhitney class of the tangent bundle $T Y_{4}$ of the 4manifold $Y_{4}$. Because the theory can be defined in this way on a nonspin manifold without imposing any conditions on bundles for the background gauge field, this is an alternate way to see that the theory describes a physical system of bosons.

[108] D. Gaiotto, A. Kapustin, Z. Komargodski, and N. Seiberg, Theta, Time Reversal and Temperature, J. High Energy Phys. (2017) 091.

[109] M. Guo, P. Putrov, and J. Wang, Time Reversal, $\mathrm{SU}(n)$ Yang-Mills and Cobordisms: Interacting Topological Superconductors/Insulators and Quantum Spin Liquids in 3+1D, Ann. Phys. (Amsterdam) 394, 244 (2018).

[110] This symmetry is usually denoted as $\mathcal{C} \mathcal{T}$ symmetry in the literature because, in the Dirac fermion representation, the $Z_{2}^{T}$ symmetry also flips the charge. The theory is also invariant with the usual time-reversal symmetry $\mathcal{T}$ and parity symmetry $\mathcal{P}$. However, these two symmetries are not relevant to our constructions.

[111] A. Kapustin and R. Thorngren, Topological Field Theory on a Lattice, Discrete Theta-Angles and Confinement, Adv. Theor. Math. Phys. 18, 1233 (2014).

[112] O. Aharony, N. Seiberg, and Y. Tachikawa, Reading between the Lines of Four-Dimensional Gauge Theories, J. High Energy Phys. (2013) 115.

[113] We thank Nathan Seiberg for a crucial discussion that directed us to the results of this section.

[114] For more details, see, e.g., the Appendix in Ref. [115].

[115] M. Cheng, Microscopic Theory of Surface Topological Order for Topological Crystalline Superconductors, Phys. Rev. Lett. 120, 036801 (2018). 
[116] J. L. Cardy, Is There a c Theorem in Four-Dimensions?, Phys. Lett. B 215, 749 (1988).

[117] The end point of the critical line may also be an interesting critical point, which may be related to the phenomenon of symmetric mass generation in 2+1-D [118].

[118] Y.-Z. You, Y.-C. He, C. Xu, and A. Vishwanath, Symmetric Fermion Mass Generation as Deconfined Quantum Criticality, Phys. Rev. X 8, 011026 (2018).

[119] A. Kitaev, Homotopy-Theoretic Approach to SPT Phases in Action: 16 Classification of Three-Dimensional Superconductors (2015).

[120] L. Fidkowski, X. Chen, and A. Vishwanath, Non-Abelian Topological Order on the Surface of a 3D Topological Superconductor from an Exactly Solved Model, Phys. Rev. X 3, 041016 (2013).

[121] L. Fu and C.L. Kane, Superconducting Proximity Effect and Majorana Fermions at the Surface of a Topological Insulator, Phys. Rev. Lett. 100, 096407 (2008).

[122] L. Fidkowski and A. Kitaev, Effects of Interactions on the Topological Classification of Free Fermion Systems, Phys. Rev. B 81, 134509 (2010).

[123] This symmetry is usually denoted as $\mathcal{C} \mathcal{T}$ symmetry in the literature because it also involves a particle-hole transformation.

[124] As written in Eq. (62), the theory also has the usual timereversal symmetry $\mathcal{T}$, which does not flip the $U(1)$ charge, as well as the parity symmetry $\mathcal{P}$. In this construction, the $\mathcal{T}$ and $\mathcal{P}$ symmetries are not important.

[125] D. Gaiotto, A. Kapustin, N. Seiberg, and B. Willett, Generalized Global Symmetries, J. High Energy Phys. 15 (2015) 172.

[126] Integrating out the fermion will generate an $S O(3) \Theta$ angle at $6 \pi$, and the $\Theta$ angle is $12 \pi$ once we restrict ourselves to the $S U(2)$ gauge bundle.

[127] C. Wang and T. Senthil, Boson Topological Insulators: A Window into Highly Entangled Quantum Phases, Phys. Rev. B 87, 235122 (2013).

[128] E. Witten, The "Parity" Anomaly on an Unorientable Manifold, Phys. Rev. B 94, 195150 (2016).

[129] Note that $w_{1}^{T Y}=1 \bmod 2$ only on a nonorientable manifold.

[130] Y.-Z. You, Z. Bi, A. Rasmussen, M. Cheng, and C. Xu, Bridging Fermionic and Bosonic Short Range Entangled States, New J. Phys. 17, 075010 (2015).

[131] M. A. Metlitski, S-Duality of U(1) Gauge Theory with $\theta=\pi$ on Non-orientable Manifolds: Applications to Topological Insulators and Superconductors, arXiv: 1510.05663.

[132] A. Kapustin, Symmetry Protected Topological Phases, Anomalies, and Cobordisms: Beyond Group Cohomology, arXiv:1403.1467.

[133] A. Kapustin, Bosonic Topological Insulators and Paramagnets: A View from Cobordisms, arXiv:1404.6659.

[134] This $Z_{2}^{T}$ transformation commutes with the $S U(2)$ gauge rotation.

[135] This $\tilde{Z}_{2}^{T}$ transformation does not commute with the $S U(2)$ gauge transformation. However, it commutes with the $S O(6)$ global symmetry.
[136] One would think that, because of the gauge transformation, the $\mathcal{T}^{2}$ of the spectator is actually meaningless. This is true if we only have a spin-1/2 boson in our theory. However, we also have adjoint fermion matter with fixed timereversal transformation. The $\mathcal{T}^{2}$ for the spectator has a physical implication in this case.

[137] X. Chen, Y.-M. Lu, and A. Vishwanath, Symmetry-Protected Topological Phases from Decorated Domain Walls, Nat. Commun. 5, 3507 (2014).

[138] M. A. Metlitski, C. L. Kane, and M. P. A. Fisher, Bosonic Topological Insulator in Three Dimensions and the Statistical Witten Effect, Phys. Rev. B 88, 035131 (2013).

[139] L. Zou, C. Wang, and T. Senthil, Symmetry Enriched U(1) Quantum Spin Liquids, Phys. Rev. B 97, 195126 (2018).

[140] We add a star as a reminder that the theory has a specific choice of spectator field.

[141] An example of such low-energy candidate theories, a $C P^{1}$ state with confinement and chiral symmetry breaking, is discussed in Ref. [59].

[142] N. Seiberg and E. Witten, Electric-Magnetic Duality, Monopole Condensation, and Confinement in $n=2$ Supersymmetric Yang-Mills Theory, Nucl. Phys. B426, 19 (1994).

[143] An easy consistency check is the $a$ theorem. As we introduced in Eqs. (54) and (55), the quantity $a$ is a universal property of every 4D CFT. It is known that $a$ is a monotonic decreasing function under renormalization group flow, namely, $a_{\mathrm{UV}}>a_{\mathrm{IR}}$ [116]. The UV theory for the $S U(2)+N_{f}^{A}=1$ theory is free $S U(2)$ Yang-Mills theory with three decoupled free Dirac fermions. For free theories, we know the simple formula for the $a$ value. Therefore, the UV value of $a$ for the adjoint $S U(2)$ theory is $a_{\mathrm{UV}}=3 \times 11+62 \times\left(2^{2}-1\right)=219$, which is indeed larger than the $a$ value of a single Dirac fermion, $a_{\text {Dirac }}=11$. Hence, our proposed IR theory is consistent with the $a$-theorem conjecture.

[144] From a formal point of view, we extend the background gauge fields but not the dynamical d.o.f. to the higherdimensional bulk. The difference between two different such extensions is described by a topological action in terms of these background gauge fields. The boundary theory by itself is not gauge invariant, but its combination with the bulk action is gauge invariant.

[145] V. P. Nair, Quantum Field Theory: A Modern Perspective (Springer, New York, 2005), p. 557.

[146] Y. Q. Qin, Y.-Y. He, Y.-Z. You, Z.-Y. Lu, A. Sen, A. W. Sandvik, C. Xu, and Z. Y. Meng, Duality between the Deconfined Quantum-Critical Point and the Bosonic Topological Transition, Phys. Rev. X 7, 031052 (2017).

[147] However, we also need to postulate an additional decoupled gapped sector in which there is no such dynamical enhancement. Nevertheless, as the free Dirac sector is decoupled, we can ask about the realization of the $Z_{8}$ on this gapless sector. The more correct assumption then is that the $Z_{8}$ is dynamically enhanced to $U(1)$ in this decoupled sector.

[148] E. Witten, An SU(2) Anomaly, Phys. Lett. B 117, 324 (1982).

[149] The normalization is that the magnetic flux coming out of a single $S O(3)$ monopole is $2 \pi$. 
[150] Y. Gu and X.-L. Qi, Axion Field Theory Approach and the Classification of Interacting Topological Superconductors, arXiv:1512.04919.

[151] Notice that this event is not allowed in the pure $S U(2)$ bundle because the minimal flux unit is twice that of the $S O(3)$ bundle.

[152] In practice, we can take the first part of Eq. (97) involving the $S O(3)$ gauge field and then restrict it to a $U(1)$ subgroup. Inserting a space-time event as described here, the result of the integral will be 4 instead of 8 .

[153] J. Wang, X.-G. Wen, and E. Witten, Symmetric Gapped Interfaces of SPT and SET States: Systematic Constructions, Phys. Rev. X 8, 031048 (2018).

[154] Preserving the 1-form symmetry means the "physical" loops are tensionful.

[155] We thank Yuji Tachikawa for clarifying this point in a private conversation.

[156] Lattice translation may or may not be present, and this makes no difference for this discussion.

[157] C. Wang, Bound States of Three Fermions Forming Symmetry-Protected Topological Phases, Phys. Rev. B 91, 245124 (2015).

[158] We can fractionalize the boson as $b \sim\left(e_{1} \partial_{x} e_{2}-e_{2} \partial_{x} e_{1}\right)$. The time-reversal transformation on the $e$ 's is $\mathcal{T}: e \rightarrow$ $i \sigma^{y} e$.
[159] An alternate construction of the same phase is to start with a standard deconfined $Z_{4}$ gauge theory and throw out all particles with odd $Z_{4}$ charge. This construction builds a $\left(Z_{2}\right)_{1}$ symmetry into the theory associated with the $Z_{4}$-flux loop with even flux. This loop does not braid nontrivially with any other excitation and has line tension. However, it is fractionalized into two odd flux loops, which themselves braid with phase $\pi$ with the particle with even $Z_{4}$ charge.

[160] In general, for $2 n$ Majorana zero modes, they form a vector representation of an $S O(2 n)$ group, and they host $2^{n}$-dimensional Hilbert space. This Hilbert space can always be decomposed into left- and right-handed spinor representations of the $S O(2 n)$ symmetry.

[161] This is true for all $S O(4 \mathbb{Z})$ groups.

[162] We have used the fact that, for the $S U(N)$ group, $\operatorname{Tr}_{A} T^{a} T^{b}=N \delta^{a b}$ and $\operatorname{Tr}_{f} T^{a} T^{b}=\frac{1}{2} \delta^{a b}$.

[163] We can check this in an explicit way. The 15 components of the Dirac fermion, by decomposing into Majorana fermions, can have at most $S O(30)$ flavor symmetries. We can explicitly check that there is only one generator in $S O(30)$ that commutes with all the $S U(4)$ generators in the adjoint representation. This generates an $S O(2)$ or a $U(1)$ global symmetry. 